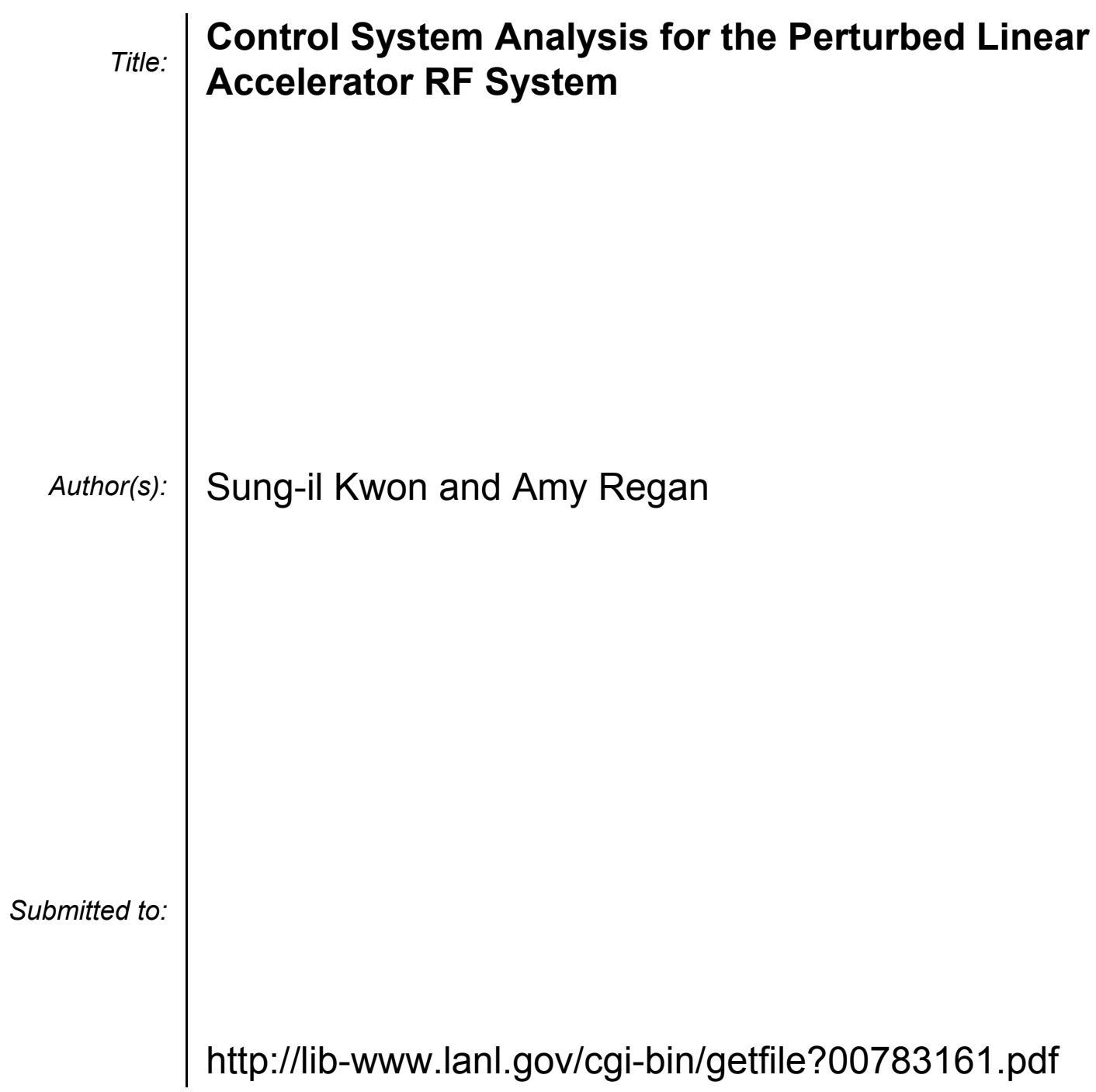




\title{
Control System Analysis for the Perturbed Linear Accelerator RF System
}

\author{
Sung-il Kwon and Amy Regan \\ SNS-2, RF Technology Group \\ SNS Division, Los Alamos National Laboratory
}

\begin{abstract}
This paper addresses the modeling problem of the linear accelerator RF system in SNS. Klystrons are modeled as linear parameter varying systems. The effect of the high voltage power supply ripple on the klystron output voltage and the output phase is modeled as an additive disturbance. The cavity is modeled as a linear system and the beam current is modeled as the exogenous disturbance. The output uncertainty of the low level RF system which results from the uncertainties in the RF components and cabling is modeled as multiplicative uncertainty. Also, the feedback loop uncertainty and digital signal processing signal conditioning subsystem uncertainties are lumped together and are modeled as multiplicative uncertainty. Finally, the time delays in the loop are modeled as a lumped time delay. For the perturbed open loop system, the closed loop system performance, and stability are analyzed with the PI feedback controller.
\end{abstract}

Index Terms - spallation neutron source, feedback control, multiplicative uncertainty, additive uncertainty, exogenous disturbance, $\mu$ analysis and synthesis, $H_{\infty}$ control, robust performance

Corresponding author: SNS-2, RF Technology Group, SNS DIVISION, Los Alamos National Laboratory, Mail Stop H827, P.O.Box 1663, Los Alamos, NM 87544, USA. email: skwon@lanl.gov, fax:665-2818

Date : 06-05-2001 


\section{INTRODUCTION}

The Spallation Neutron Source (SNS) Linac to be built at Oak Ridge National Laboratory (ORNL) consists of a combination of low energy normal conducting (NC) accelerating structures as well as higher energy superconducting RF (SRF) structures. The RF Controls team at Los Alamos National Laboratory is responsible for the RF Controls systems for both the NC and SRF portions of the linac. In order to efficiently provide a working control system, the RF Controls team has performed a lot of modeling.

The purpose of modeling is to investigate the various cavity configurations in order to provide the correct requirements for the control system hardware. We use modeling as a way to specify RF components; verify system design and performance objectives; optimize control parameters; and to provide further insight into the RF control system operation. At LANL this sort of modeling has been utilized and proven on a number of pulsed and $\mathrm{CW}$ accelerators for at least the past ten years.

A main part of a linear accelerator RF system is the cascade of a nonlinear klystron and a linear cavity. In order to investigate the system characteristics, a MATLAB/SIMULINK model has been constructed and an enormous number of simulations have been performed. When the cascade of a klystron and a cavity is not a single one, it is very difficult to study the performance of the total number of cascades through simulation. In SNS, the linear accelerator section is composed of a normal conducting subsection and a superconducting subsection. The normal conducting subsection is composed of $1 \mathrm{RFQ}, 6 \mathrm{DTL}$ tanks, 4 CCL modules; the superconducting section is composed 33 low beta SRF cavities, 48 high beta cavities. The RFQ is driven by a $2.5 \mathrm{MW}$ klystron, each DTL tank is driven by a $2.5 \mathrm{MW}$ klystron, each CCL module is driven by a $5.0 \mathrm{MW}$ klystron, and each SRF cavity is driven by a $550 \mathrm{~kW}$ klystron. Hence, total cascades of a klystron and a cavity are 92 . Also, each cascade of a klystron and a cavity is controlled by a low level RF controller and so, at an extreme, 92 different controllers are necessary. For syntheses of controllers and analyses of closed loop systems and open loop systems, modern control theory that provides the systematic tools for analysis and synthesis is applied. In order to apply the modern control theory, it is inevitable to obtain a linearized klystron model for a nonlinear klystron at the specific operating point of the klystron. Hence, the cascade of a klysron and a cavity is approximated by a linear system.

In the real world, uncertainties and disturbances are unavoidable. In a linear accelerator RF system, there are several sources of the uncertainties and the disturbances. For a klystron, the major disturbance source is the high voltage power supply (HVPS) ripple. This disturbance affects both the output amplitude and the output phase of a klystron. A model for the ripple is necessary to investigate the klystron performance. For a SRF cavity, the major disturbances on the cavity characteristics are the Lorentz Force Detuning and the microphonics. Also, the changes of RF parameters should be investigated and be included in the model. In the low level RF control system, many RF components are used and these components are not ideal and have their own uncertainties and latencies. Also, feedback loop time delay, waveguide time delay, and other time delays are modeled. All of these uncertainties, disturbances, and time delays, are modeled as either multiplicative uncertainties, additive uncertainties, or exogenous disturbances.

Finally, for perturbed system models, low level RF controllers are synthesized by applying modern control theory such as $\mathrm{H}_{2}$ control, $H_{\infty}$ control, loop shaping control, $H_{\infty}$ based PI control. Closed loop system stability and performance are analyzed.

The structure of this paper is as follows: In the next section a nonlinear klystron is modeled. A normalization process of the amplitude saturation curve and the phase saturation curve based on the measured input power-output power data and input power-output phase data. A mathematical nonlinear klystron model is obtained by approximating the amplitude saturation curve and the phase saturation curve using least square sense polynomial curve fitting. Then, a linearized model of the klystron around an operating point is obtained. The HVPS ripple effect is then modeled as both an output multiplicative uncertainty and an exogenous disturbance. In section III, a SRF cavity and a normal conducting cavity are 
modeled. Also, the perturbed model for a SRF cavity due to the variations of RF parameters is investigated. In section IV, the feedback loop uncertainties and forward loop uncertainties are modeled as multiplicative uncertainties. Also, time delays are lumped together and are modeled as a multiplicative uncertainty. In section $\mathrm{V}$, all perturbed models are integrated and a perturbed linear accelerator RF system is obtained. 


\section{KLYSTRON MODELS}

In SNS, the normal conducting linac part consists of 1 RFQ, 6 DTL tanks, 4 CCL modules. The superconducting linac part consists of 81 SRF cavities. Each tank, module, and SRF cavity produces a different level of energy and transfers a different amount energy to the beam. For example, the following table shows the required RF parameters in 6 DTL tanks.

Table 2.1 SNS DTL Tank Parameters

\begin{tabular}{|c|c|c|c|c|c|c|c|c|c|c|c|}
\hline Tank & $\begin{array}{c}\Delta \mathrm{W} \\
(\mathrm{MeV})\end{array}$ & $\begin{array}{c}\mathrm{P}_{\mathrm{B}} \\
(\mathrm{MW})\end{array}$ & $\begin{array}{c}\phi_{\mathrm{s}} \\
(\mathrm{deg})\end{array}$ & $\begin{array}{c}\mathrm{E}_{0} \mathrm{~T} \\
(\mathrm{MV} / \mathrm{m})\end{array}$ & $\begin{array}{c}\mathrm{U} \\
(\mathrm{J})\end{array}$ & $\mathrm{Q}_{0}$ & $\begin{array}{c}\mathrm{P}_{\mathrm{C}} \\
(\mathrm{MW})\end{array}$ & $\beta$ & $\mathrm{Q}_{\text {Ext }}$ & $\mathrm{Q}_{\mathrm{L}}$ & $\begin{array}{c}\psi \\
(\mathrm{deg})\end{array}$ \\
\hline 1 & 5.023 & 0.171 & -36 & 1.495 & 4.78 & 35,887 & 0.337 & 1.508 & 23,803 & 14,311 & -8.4 \\
\hline 2 & 15.315 & 0.521 & -25 & 2.787 & 16.51 & 39,919 & 1.046 & 1.498 & 26,646 & 15,980 & -5.3 \\
\hline 3 & 16.951 & 0.576 & -25 & 2.958 & 21.69 & 42,122 & 1.302 & 1.442 & 29,204 & 17,247 & -4.8 \\
\hline 4 & 16.790 & 0.571 & -25 & 2.889 & 21.15 & 42,472 & 1.259 & 1.453 & 29,222 & 17,311 & -4.9 \\
\hline 5 & 15.916 & 0.571 & -25 & 2.789 & 21.36 & 42,526 & 1.270 & 1.450 & 29,338 & 17,361 & -4.9 \\
\hline 6 & 14.333 & 0.487 & -35 & 2.761 & 21.48 & 42,412 & 1.281 & 1.380 & 30,728 & 17,818 & -6.4 \\
\hline
\end{tabular}

Since each klystron supplies RF power to each DTL tank, the operating point of each klystron is different from one klystron to another klystron. This requires the parameterization of the operating point of a klystron with respect to the required power (sum of beam power, $P_{B}$, and copper power loss, $P_{C}$ ). A klystron can be expressed as the cascade of a linear subsystem and a nonlinear output subsystem. The linear subsystem represents the $3 \mathrm{~dB}$ bandwidth of the klystron and the constant gain. The nonlinear output subsystem represents the amplitude saturation curve and the phase saturation curve of the klystron. The amplitude saturation curve and the phase saturation curve are represented by the table of measured inputoutput data. Given measured data, the amplitude saturation curve and the phase saturation curve are approximated to analytic, mathematical equations by curve fittings in the least square sense. Basis of the curve fitting can be chosen from the function such as Bessel function, exponential, power series and the choice is closely related to the simplicity of the modeling. Here, power series basis is chosen.

\section{II-A. Amplitude Saturation Curve and Phase Saturation Curve Normalization}

In the linac part of the SNS, $62.5 \mathrm{MW}$ klystrons are used in DTL tanks, 4 5.0 MW klystrons are used in 4 CCL modules, and $81550 \mathrm{~kW}$ klystrons are used for SRF cavities. For each klystron type, the normalizations for the amplitude saturation curve and the phase saturation curve are needed for the modeling of the klystron.

Table 2.2 VKP-8290A amplitude saturation data

\begin{tabular}{|c|c|c|}
\hline Input Power $(\mathrm{W})$ & Output Power $(\mathrm{MW})$ & Gain $(\mathrm{dB})$ \\
\hline 6.7000 & 0.1600 & 43.7805 \\
\hline 11.4799 & 0.5933 & 47.1334 \\
\hline 17.1789 & 1.0000 & 47.6500 \\
\hline 22.8780 & 1.5467 & 48.2999 \\
\hline 31.5186 & 2.0000 & 48.0246 \\
\hline 34.2762 & 2.1333 & 47.9400 \\
\hline 39.5753 & 2.3333 & 47.7049 \\
\hline 45.6744 & 2.4733 & 47.3360 \\
\hline 57.0726 & 2.6000 & 46.5855 \\
\hline 67.0000 & 2.6050 & 45.8973 \\
\hline 91.2671 & 2.6000 & 44.5466 \\
\hline
\end{tabular}




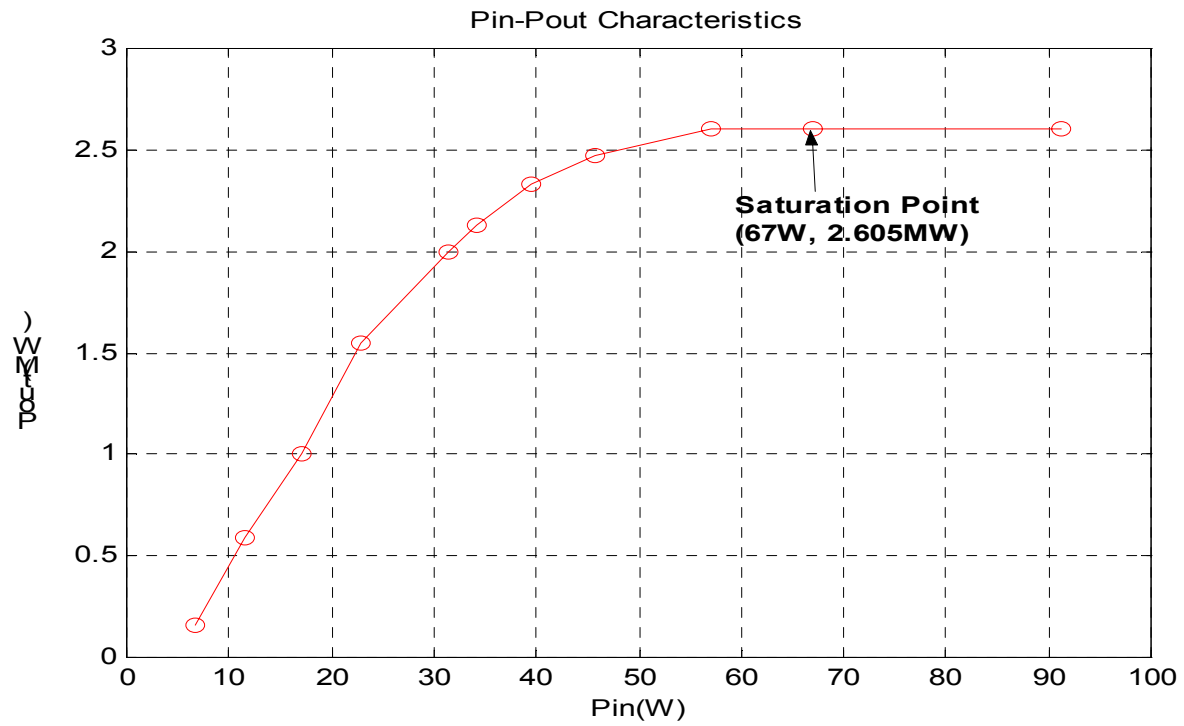

Figure 2.1 Amplitude Saturation Curve

To understand the normalizations, a $2.5 \mathrm{MW}$ klystron, VKP-8290A is taken as an example. Table 3.1 shows the measured input power-output power relation of a VKP-8290A klystron and figure 2.1 shows its amplitude saturation curve. At the input power $67 \mathrm{~W}$, the output power reaches its saturation, $2.605 \mathrm{MW}$. The amplitude saturation curve of the klystron can be normalized in such a way that the input power-output power pair $\left(P_{\text {in }}^{\text {sat }}, P_{\text {out }}^{\text {sat }}\right)=(67 \mathrm{~W}, 2.605 \mathrm{MW})$ is mapped to $(1,1)$ pair and other pairs $\left(P_{\text {in }}, P_{\text {out }}\right)$ are mapped to $\left(\frac{P_{\text {in }}}{P_{\text {in }}^{\text {sat }}}, \frac{P_{\text {out }}}{P_{\text {out }}^{\text {sat }}}\right)$ pairs. Also, from the rule, $V=\sqrt{2 R_{O} P}$, the normalized input voltage-output voltage mapping can be obtained. Here, $R_{o}$ is the transmission line characteristic impedance. These mappings yield the normalization as given in table 2.3 and figures 2.2-2.3. In figure 2.2 and figure 2.3, two points are line-interpolated, which may result in the inaccurate input-output relation. The normalized amplitude saturation curves as shown in figures 2.2-2.3 make it easy to obtain the input-output relation for the operation of the klystron at a specified power control margin.

Suppose the klystron is to be operated at $x \%$ power control margin. Then, the corresponding operating output power and operating output voltage of the klystron in the normalized amplitude saturation curves are given by

$$
\begin{aligned}
P_{\text {out }}^{N O P} & =\frac{1.0}{1.0+x / 100}, \\
V_{\text {out }}^{N O P} & =\sqrt{P_{\text {out }}^{N O P}}=\sqrt{\frac{1.0}{1.0+x / 100}} .
\end{aligned}
$$

In the unnormalized amplitude saturation curves, corresponding operating output power and voltage corresponding to $x \%$ power control margin are 


$$
\begin{gathered}
P_{\text {out }}^{O P}=P_{\text {out }}^{N O P} \cdot P_{\text {out }}^{\text {Sat }}=\frac{1.0}{1.0+x / 100} \cdot P_{\text {out }}^{\text {Sat }}, \\
V_{\text {out }}^{O P}=V_{\text {out }}^{N O P} \cdot V_{\text {out }}^{\text {Sat }}=\sqrt{\frac{1.0}{1.0+x / 100}} \cdot \sqrt{2 R_{o} P_{\text {out }}^{\text {Sat }}}
\end{gathered}
$$

where

$$
V_{\text {out }}^{\text {Sat }}=\sqrt{2 R_{o} P_{\text {out }}^{\text {Sat }}}
$$

The corresponding operating input power and operating input voltage are determined in the normalized amplitude saturation curves as shown in figure 2.2 and figure 2.3.

$$
\begin{array}{lll}
\text { Normalized Input } & \Leftarrow & \text { Normalized Output } \\
P_{\text {in }}^{N O P} & \Leftarrow & P_{\text {out }}^{N O P}=\frac{1.0}{1.0+x / 100} \\
V_{\text {in }}^{N O P} & \Leftarrow & V_{\text {out }}^{N O P}=\sqrt{\frac{1.0}{1.0+x / 100} .}
\end{array}
$$

Also, the unnormalized operating input power and operating input voltage are given by

$$
\begin{aligned}
& P_{i n}^{O P}=P_{i n}^{N O P} \cdot P_{i n}^{S a t} \\
& V_{i n}^{O P}=V_{i n}^{N O P} \cdot V_{i n}^{S a t}
\end{aligned}
$$

where

$$
V_{i n}^{S a t}=\sqrt{2 R_{o} P_{i n}^{S a t}}
$$

When the klystron is to be operated at the $25 \%$ power control margin, then the input-output relations in the normalized amplitude saturation curves are as follows. The operating output powers are

$$
P_{\text {out }}^{N O P}=\frac{1.0}{1.0+25 / 100}=0.80 \mathrm{~W}, \quad \quad P_{\text {out }}^{\text {OP }}=P_{\text {out }}^{N O P} \cdot P_{\text {out }}^{\text {Sat }}=0.80 \cdot 2.605=2.084 \mathrm{MW} .
$$

The corresponding operating output voltages are

$$
V_{\text {out }}^{N O P}=\sqrt{\frac{1.0}{1.0+25 / 100}}=0.8944 \mathrm{~V}, \quad V_{\text {out }}^{\text {OP }}=V_{\text {out }}^{N O P} \cdot \sqrt{2 R_{o} P_{\text {out }}^{\text {Sat }}}=0.8944 \cdot 16.14=14.436 \mathrm{kV}
$$

Also, the corresponding operating input power and operating input voltage are obtained from the amplitude saturation curves of power and voltage even though it is difficult to obtain the exact values from the amplitude saturation curves. The operating input powers are

$$
P_{i n}^{N O P}=0.4963 \mathrm{~W}, \quad P_{i n}^{O P}=P_{i n}^{N O P} \cdot P_{i n}^{S a t}=0.4963 \cdot 67.0=33.2521 \mathrm{~W}
$$

and the operating input voltages are

$$
V_{\text {in }}^{N O P}=0.7045 \mathrm{~V}, \quad V_{\text {in }}^{O P}=57.6646 \mathrm{~V}
$$


Table 2.3 VKP-8290A normalized amplitude saturation data

\begin{tabular}{|c|c|c|c|}
\hline $\begin{array}{c}\text { Normalized } \\
\text { Input }(\mathrm{W})\end{array}$ & $\begin{array}{c}\text { Normalized } \\
\text { Output }(\mathrm{W})\end{array}$ & $\begin{array}{c}\text { Normalized } \\
\text { Input }(\mathrm{V})\end{array}$ & $\begin{array}{c}\text { Normalized } \\
\text { Output }(\mathrm{V})\end{array}$ \\
\hline 0.1000 & 0.0614 & 0.3162 & 0.2478 \\
\hline 0.1713 & 0.2278 & 0.4139 & 0.4772 \\
\hline 0.2564 & 0.3839 & 0.5064 & 0.6196 \\
\hline 0.3415 & 0.5937 & 0.5843 & 0.7705 \\
\hline 0.4704 & 0.7678 & 0.6859 & 0.8762 \\
\hline 0.5116 & 0.8188 & 0.7153 & 0.9049 \\
\hline 0.5907 & 0.8956 & 0.7686 & 0.9464 \\
\hline 0.6817 & 0.9494 & 0.8257 & 0.9744 \\
\hline 0.8518 & 0.9981 & 0.9229 & 0.9990 \\
\hline 1.0000 & 1.0000 & 1.0000 & 1.0000 \\
\hline 1.3622 & 0.9981 & 1.1671 & 0.9990 \\
\hline
\end{tabular}

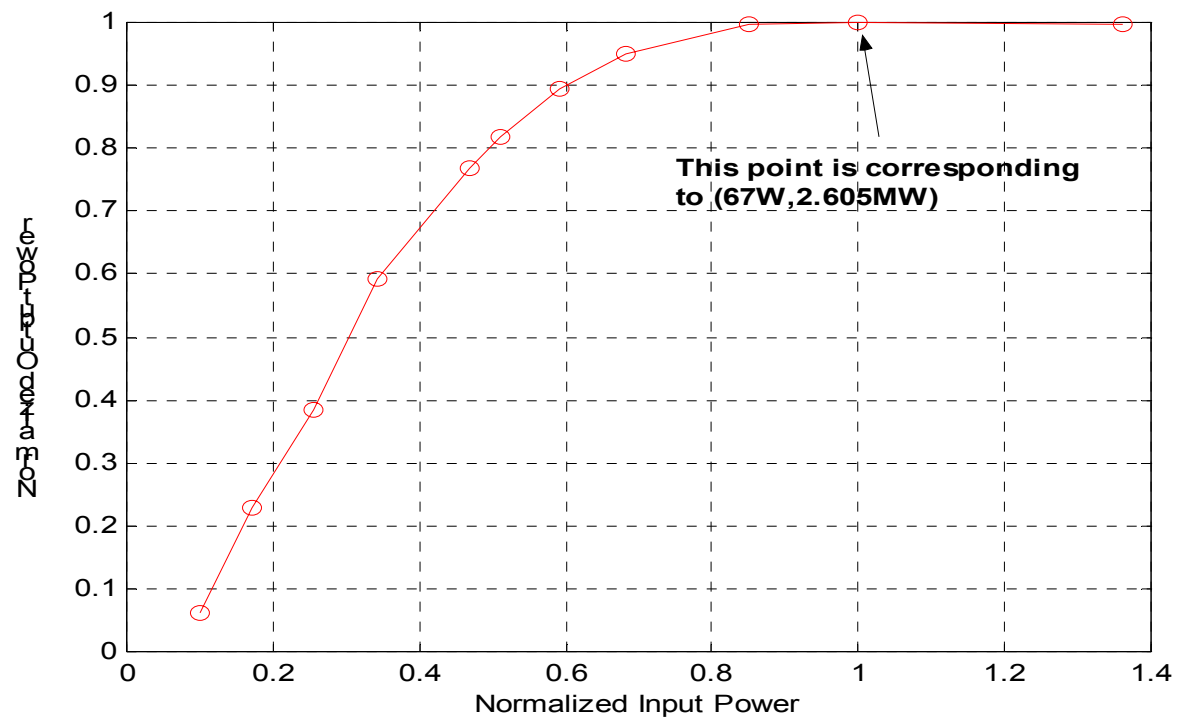

Figure 2.2 Normalized Amplitude Saturation Curve in Input Power-Output Power 


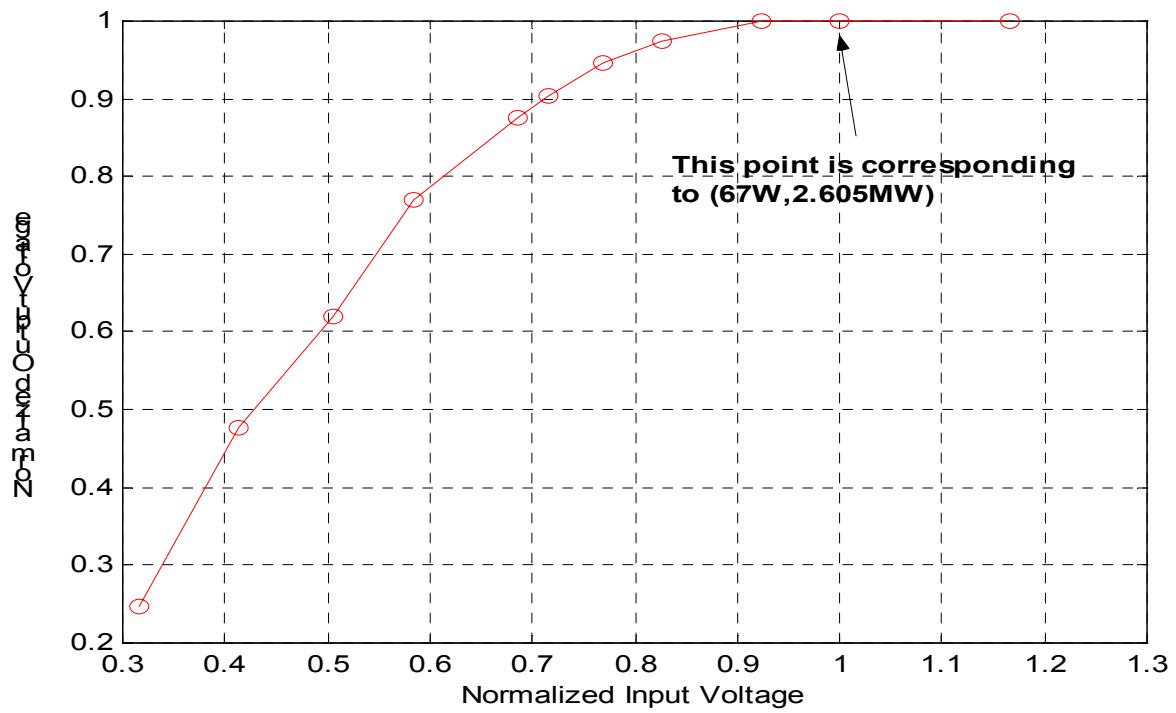

Figure 2.3 Normalized Amplitude Saturation Curve in Input Voltage-Output Voltage

The relations $\left(P_{\text {in }}^{\text {Sat }}, P_{\text {out }}^{\text {Sat }}\right),\left(V_{\text {in }}^{\text {Sat }}, V_{\text {out }}^{\text {Sat }}\right),\left(P_{\text {in }}^{N O P}, P_{\text {out }}^{N O P}\right),\left(V_{\text {in }}^{\text {NOP }}, V_{\text {out }}^{N O P}\right),\left(P_{\text {in }}^{O P}, P_{\text {out }}^{O P}\right), \quad\left(V_{\text {in }}^{O P}, V_{\text {out }}^{\text {OP }}\right)$ when the klystron is operated at the $25 \%$ power control margin are summarized in figure 2.4 .

The phase saturation curve can be also normalized. Table 2.4 and figure 2.5 show the phase saturation curve data of the VKP-8290A klystron. At the input power $6.7 \mathrm{~W}$, the phase shift is 44.3 degrees. A phase offset of 44.3 degree is introduced so that the pair ( $6.7 \mathrm{~W}, 44.3$ degrees) becomes $(6.7 \mathrm{~W}, 0.0$ degrees). Other input power-phase shift pair $\left(\mathrm{P}_{\mathrm{in},} \phi\right)$ is reduced to $\left(\mathrm{P}_{\mathrm{in}}, \bar{\phi}\right)$ where

$$
\bar{\phi}=\phi-44.3
$$

Then, the input power-offsetted phase pairs are normalized in such a way that $\left(\mathrm{P}_{\mathrm{in}}, \phi-44.3\right)$ pairs are mapped to $\left(\mathrm{P}_{\mathrm{in}} / 67.0, \phi\right.$-44.3) pairs. Also, from the rule, $V_{i n}=\sqrt{2 R_{o} P_{i n}}$, the normalized input voltageoffsetted phase shift mapping can be obtained. The normalized phase saturation curve data and the corresponding curves are shown in table 2.5 and figures 2.6-2.7, respectively. As are the cases of the amplitude saturation curves, two data points are line-interpolated. Figure 2.8 shows the schematic diagram for the normalization of the amplitude saturation curve and the normalization of the phase saturation curve. 


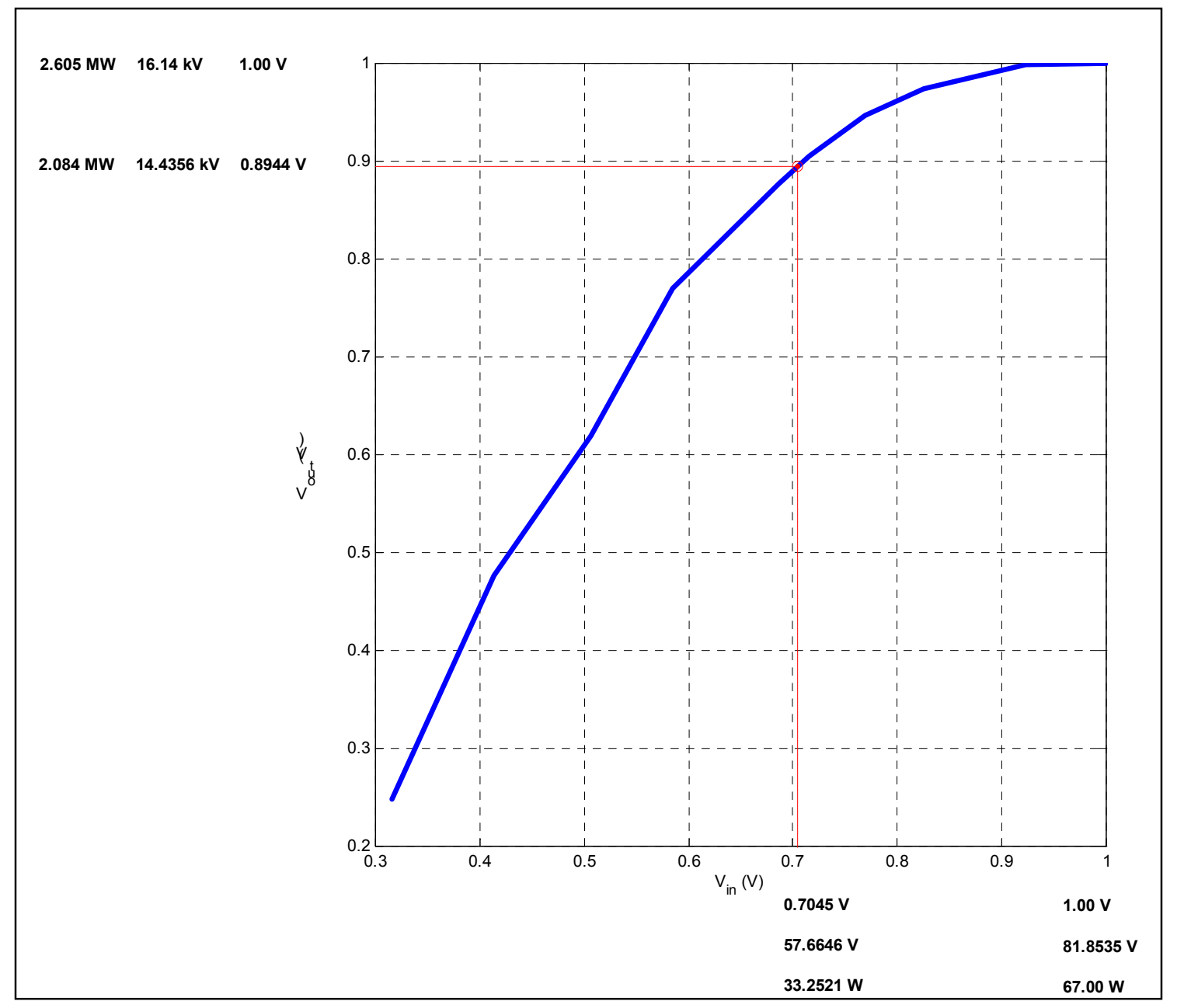

Figure 2.4 Input-Output relations in the normalized amplitude saturation curve 
Table 2.4 VKP-8290A phase saturation data

\begin{tabular}{|c|c|}
\hline Input Power (W) & Output Phase (Deg) \\
\hline 6.7000 & 44.3000 \\
\hline 8.4348 & 43.9000 \\
\hline 10.6188 & 43.6000 \\
\hline 13.3683 & 43.6000 \\
\hline 16.8296 & 43.5000 \\
\hline 21.1873 & 43.3000 \\
\hline 26.6732 & 43.0000 \\
\hline 33.5795 & 41.7000 \\
\hline 42.2741 & 40.5000 \\
\hline 53.2200 & 40.0000 \\
\hline 67.0000 & 39.2000 \\
\hline
\end{tabular}

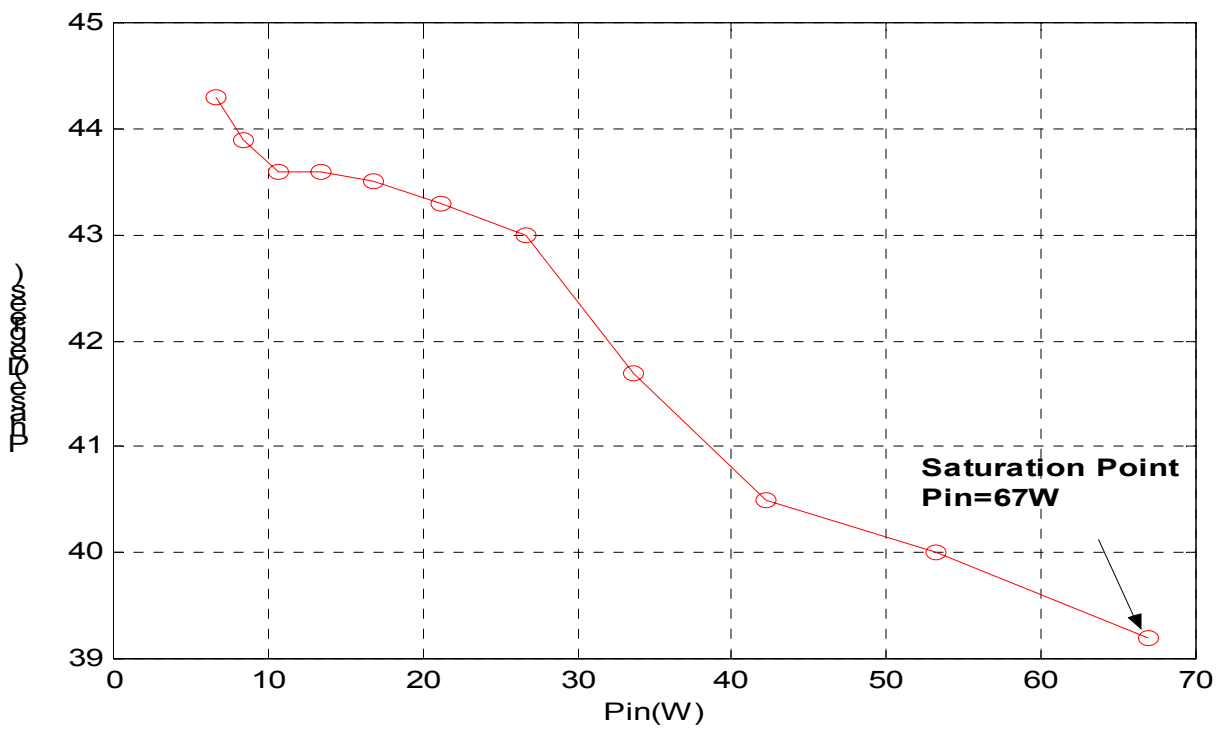

Figure 2.5 Phase Saturation Curve 
Table 2.5 VKP-8290A Normalized phase saturation data

\begin{tabular}{|c|c|c|c|}
\hline $\begin{array}{c}\text { Normalized } \\
\text { Input (W) }\end{array}$ & $\begin{array}{c}\text { Normalized } \\
\text { Phase (Deg) }\end{array}$ & $\begin{array}{c}\text { Normalized } \\
\text { Input (V) }\end{array}$ & $\begin{array}{c}\text { Normalized } \\
\text { Phase (Deg) }\end{array}$ \\
\hline 0.1000 & 0.0000 & 0.3162 & 0.0000 \\
\hline 0.1259 & -0.4011 & 0.3548 & -0.4011 \\
\hline 0.1585 & -0.6990 & 0.3981 & -0.6990 \\
\hline 0.1995 & -0.6990 & 0.4467 & -0.6990 \\
\hline 0.2512 & -0.8021 & 0.5012 & -0.8021 \\
\hline 0.3162 & -1.0027 & 0.5623 & -1.0027 \\
\hline 0.3981 & -1.3006 & 0.6310 & -1.3006 \\
\hline 0.5012 & -2.6012 & 0.7079 & -2.6012 \\
\hline 0.6310 & -3.7987 & 0.7943 & -3.7987 \\
\hline 0.7943 & -4.2972 & 0.8913 & -4.2972 \\
\hline 1.0000 & -5.0993 & 1.0000 & -5.0993 \\
\hline
\end{tabular}

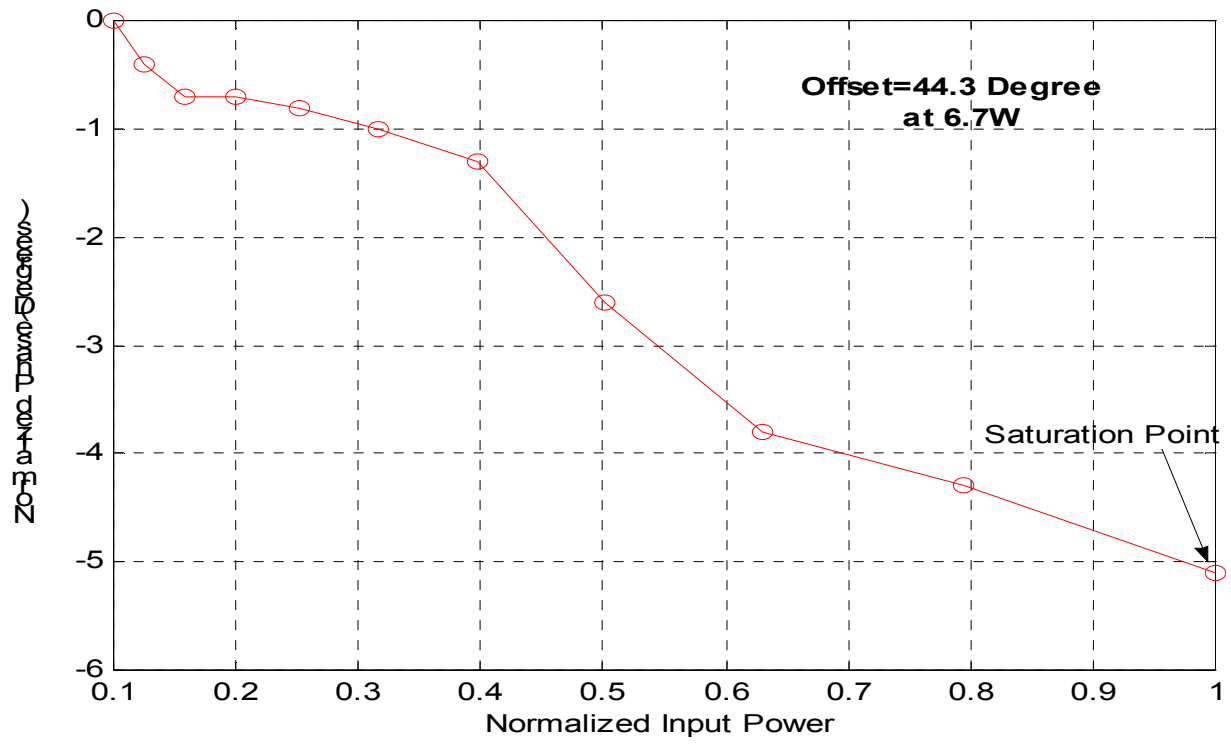

Figure 2.6 Normalized Phase Saturation Curve in Input Power-Output Phase 


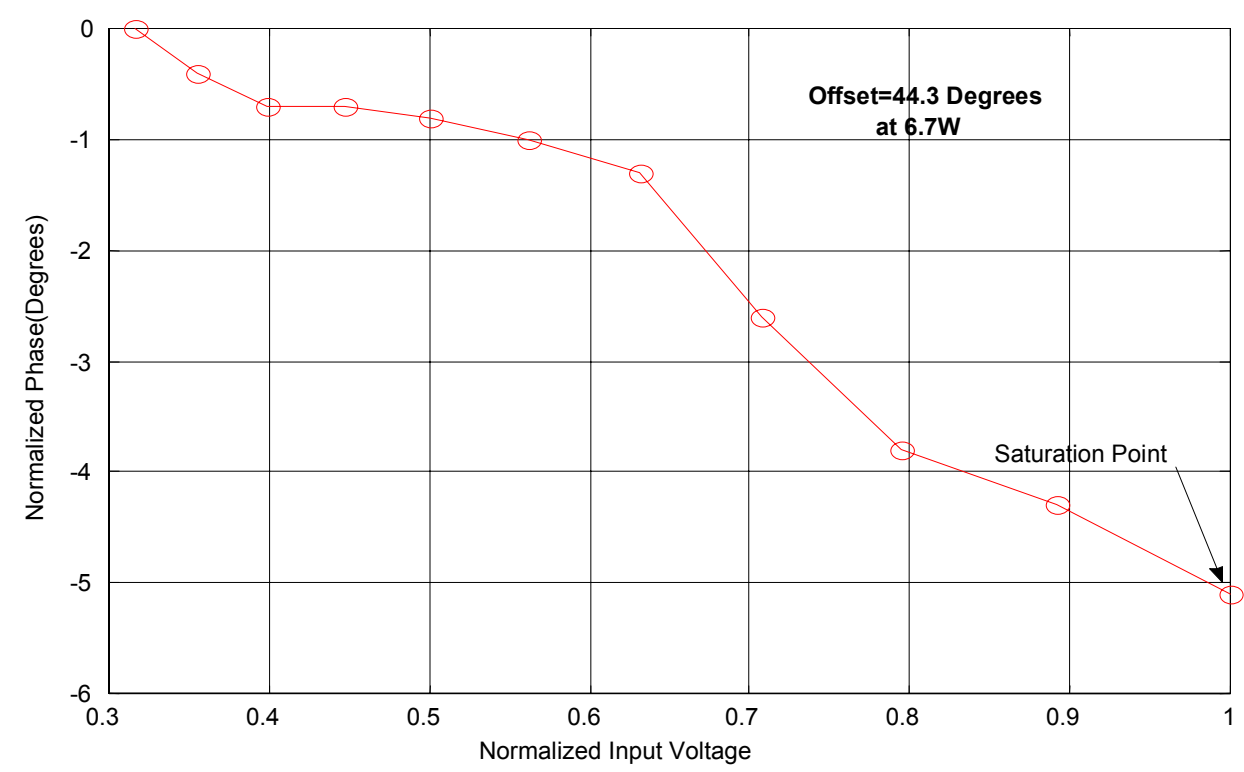

Figure 2.7 Normalized Phase Saturation Curve in Input Voltage-Output Phase

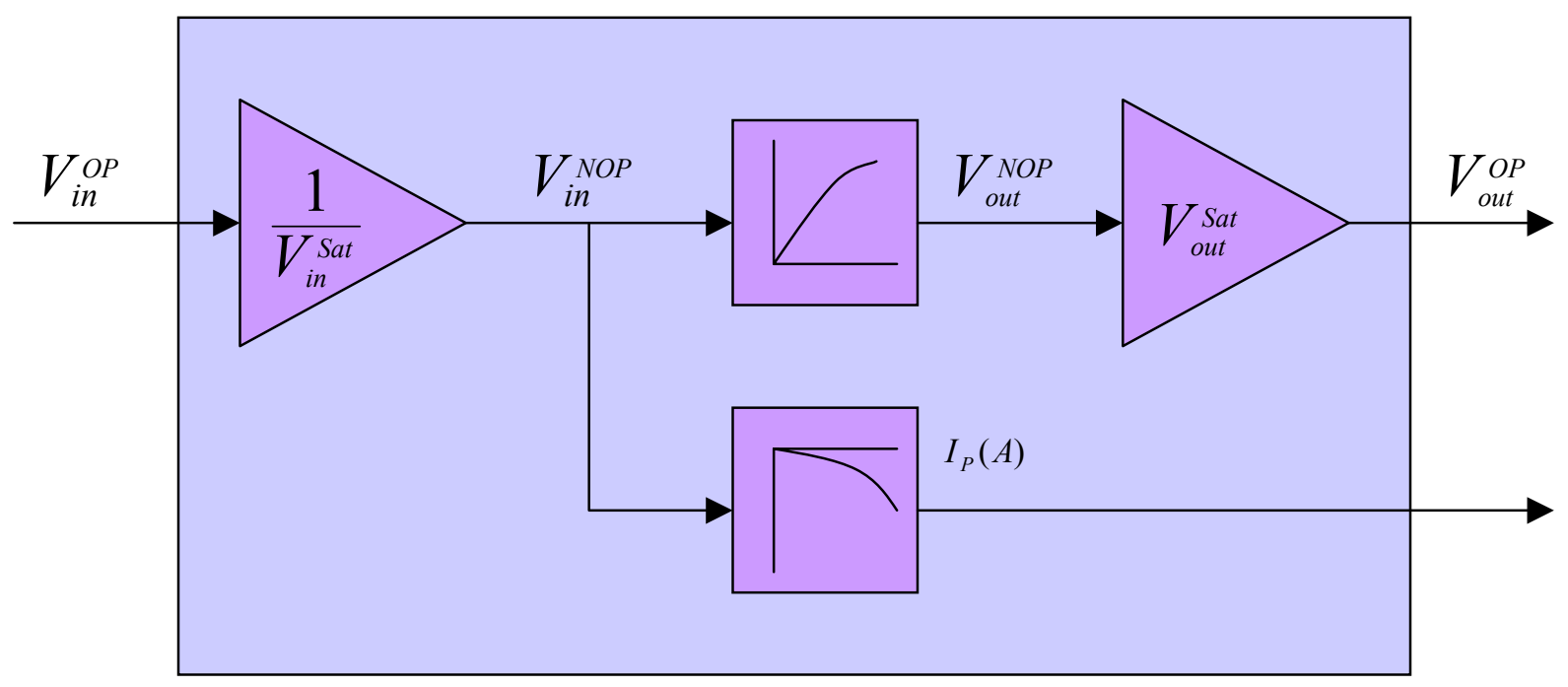

Figure 2.8 Schematic Diagram of the normalizations of the amplitude saturation curve and the phase saturation curve. 


\section{II-B. Analytic Representation of Amplitude Saturation Curve and Phase Saturation Curve}

In order to represent the amplitude saturation curve and the phase saturation curve accurately, it is necessary to measure as many as possible input power-output power pairs, and input power-output phase pairs. In general, this is impossible. Instead, it is useful to obtain analytic equations for the amplitude saturation curve and the phase saturation curve based on the finite number of measured data. For this purpose, least square sense curve fitting is of general use. For the basis of the curve fitting, Bessel function, exponential function, power series and others are considered. The basis is chosen in such a way to simplify the mathematical model of the klystron. In this note, power series are selected. In the basis of power series, the normalized amplitude saturation curve and the normalized phase saturation curve are expressed as

$$
\begin{aligned}
& y_{A}=I_{A}(A)=\sum_{i=1}^{N} \bar{c}_{i} A^{i} \\
& y_{P}=I_{P}(A)=\sum_{i=1}^{N} \bar{d}_{i} A^{i}
\end{aligned}
$$

where $A, 0.0 \leq A \leq 1.0$, is the normalized input voltage, $V_{i n}^{N O P}$, and $\bar{c}_{i}, \bar{d}_{i}, \quad i=1,2, \cdots, N$ are characteristic coefficients of the amplitude saturation and the phase saturation curves of the klystron to be obtained. Coefficients $\bar{c}_{i}, \bar{d}_{i}, i=1,2, \cdots, N$ are determined in a least square sense from the data in table 2.3 and table 2. Table 2.6 shows the coefficients $\bar{c}_{i}, \bar{d}_{i}, \quad i=1,2, \cdots, N$ and figure 2.8 and figure 2.9 show the plots of the equations (2.11) and (2.12) with respect to the normalized input voltage $A$.

Similarly, the inverse of the amplitude saturation curve and the inverse of the phase saturation curve of the klystron can be represented by power series of the output amplitude $y_{A}$ and the output phase $y_{P}$

$$
\begin{aligned}
& I_{A}^{-1}\left(y_{A}\right)=\sum_{i=1}^{N} \bar{e}_{i} y_{A}^{i} \\
& I_{P}^{-1}\left(y_{P}\right)=\sum_{i=1}^{N} \bar{f}_{i} y_{P}^{i}
\end{aligned}
$$

where $\bar{e}_{i}, \quad \bar{f}_{i}, \quad i=1,2, \cdots, N$ are characteristic coefficients of the inverse of the amplitude saturation curve and the inverse of the phase saturation curve of the klystron. Coefficients $\bar{e}_{i}, \bar{f}_{i}, \quad i=1,2, \cdots, N$ are determined in a least square sense from the data in tables 2.3 and 2.5. Table 2.7 shows the coefficients $\bar{e}_{i}$, $\bar{f}_{i}, \quad i=1,2, \cdots, N$ and figure 2.10 and figure 2.11 show plots of the equations (2.13) and (2.14) with respect to the output amplitude $y_{A}$ and the output phase $y_{P}$, respectively.

As shown in figure 2.9, with a $5^{\text {th }}$ order power series, the phase saturation curve is not fitted well, even though the amplitude saturation curve is properly fitted. Hence, the power series order is increased to 7 . Table 2.8 shows the coefficients of the power series of amplitude saturation curve and the phase saturation curve and figures 2.12 and 2.13 show the plots of curve fitting. Table 2.9 shows the coefficients of the power series of inverse amplitude saturation curve and the inverse phase saturation curve and figure 2.14 and figure 2.15 show the plots of curve fitting. 
Table 2.6 Klystron Parameters

\begin{tabular}{|c|c|c|c|}
\hline $\bar{c}_{1}$ & -1.9104 & $\bar{d}_{1}$ & 0.1568 \\
\hline $\bar{c}_{2}$ & 14.2115 & $\bar{d}_{2}$ & -1.2518 \\
\hline $\bar{c}_{3}$ & -21.7856 & $\bar{d}_{3}$ & 3.4346 \\
\hline $\bar{c}_{4}$ & 13.5672 & $\bar{d}_{4}$ & -4.2600 \\
\hline $\bar{c}_{5}$ & -3.0796 & $\bar{d}_{5}$ & 1.8326 \\
\hline
\end{tabular}

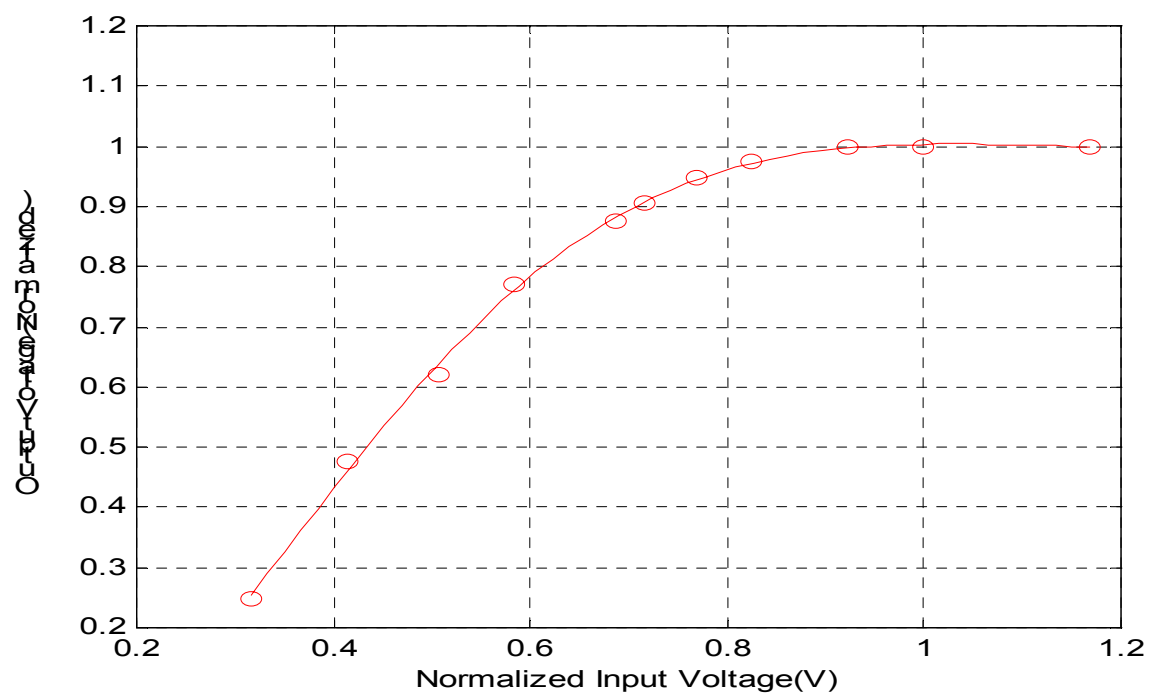

Figure 2.8 Curve Fitting of Normalized Amplitude Saturation Curve ( $5^{\text {th }}$ order curve fitting)

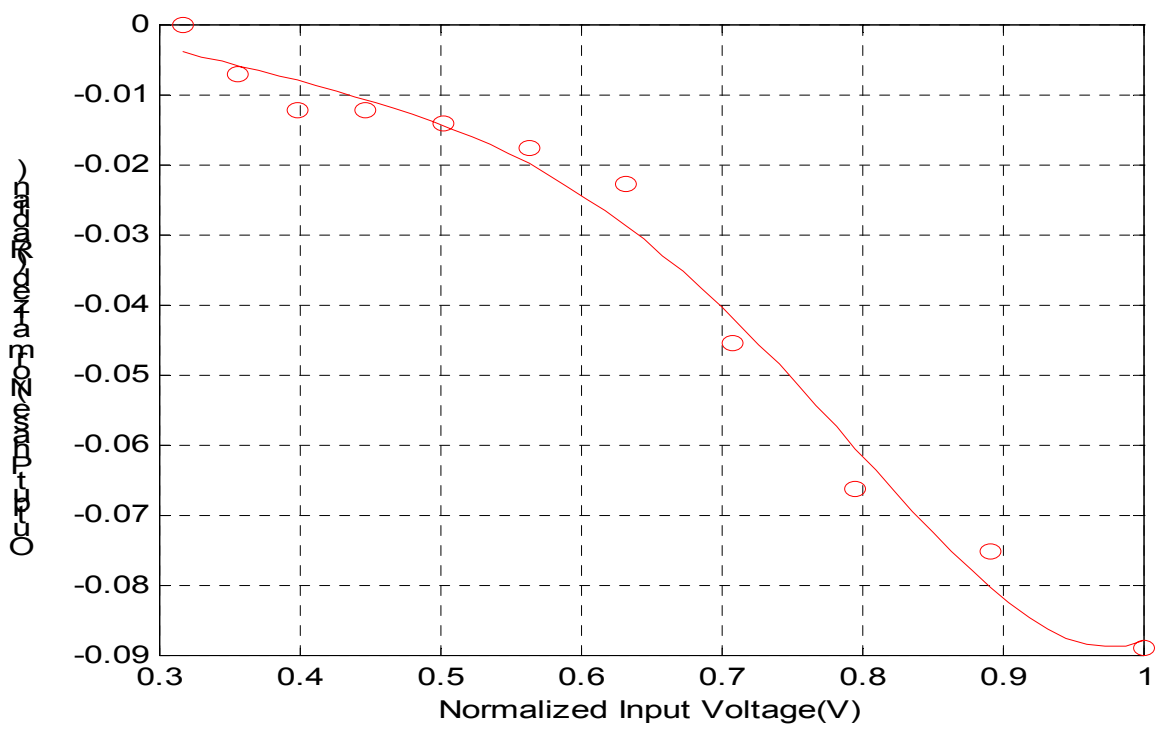

Figure 2.9 Curve Fitting of Normalized Phase Saturation Curve ( $5^{\text {th }}$ order curve fitting) 
Table 2.7 Klystron Parameters

\begin{tabular}{|c|c|c|c|}
\hline $\bar{e}_{1}$ & 5.2048 & $\bar{f}_{1}$ & -52.8462 \\
\hline $\bar{e}_{2}$ & -29.9409 & $\bar{f}_{2}$ & $-1.4873 \mathrm{e}+003$ \\
\hline $\bar{e}_{3}$ & 75.4209 & $\bar{f}_{3}$ & $-1.7521 \mathrm{e}+004$ \\
\hline $\bar{e}_{4}$ & -82.2812 & $\bar{f}_{4}$ & $-5.8398 \mathrm{e}+004$ \\
\hline $\bar{e}_{5}$ & 32.6127 & $\bar{f}_{5}$ & $1.0913 \mathrm{e}+005$ \\
\hline
\end{tabular}

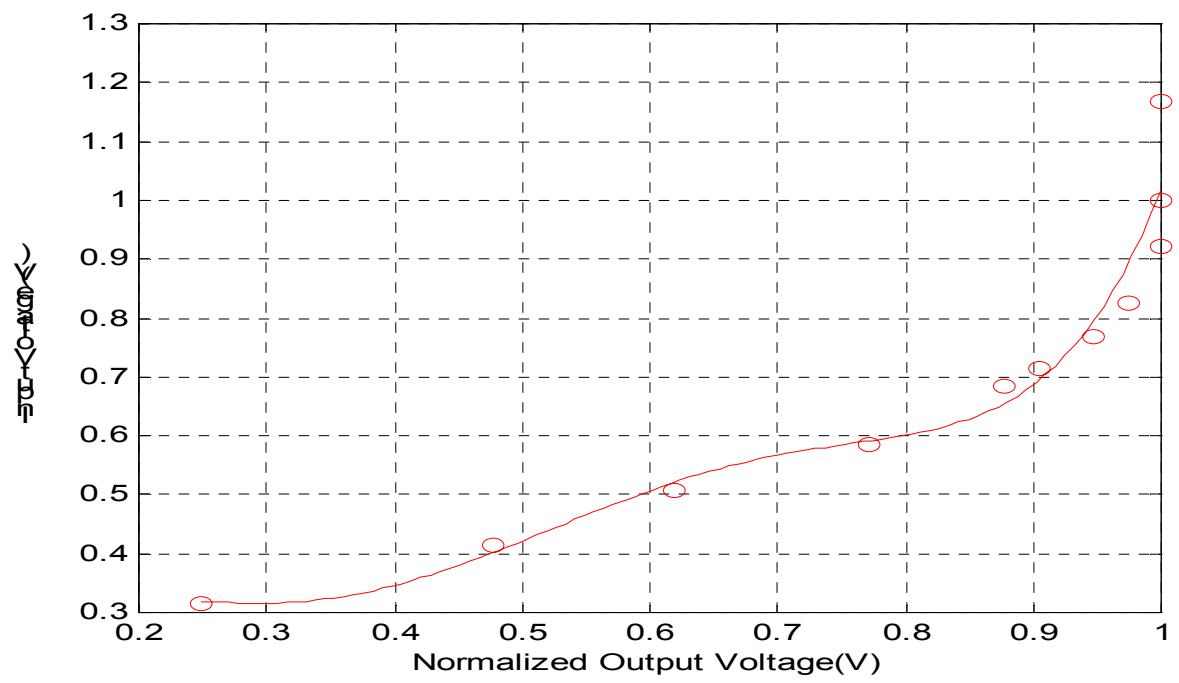

Figure 2.10 Curve Fitting of Inverse of Normalized Amplitude Saturation Curve ( $5^{\text {th }}$ order curve fitting)

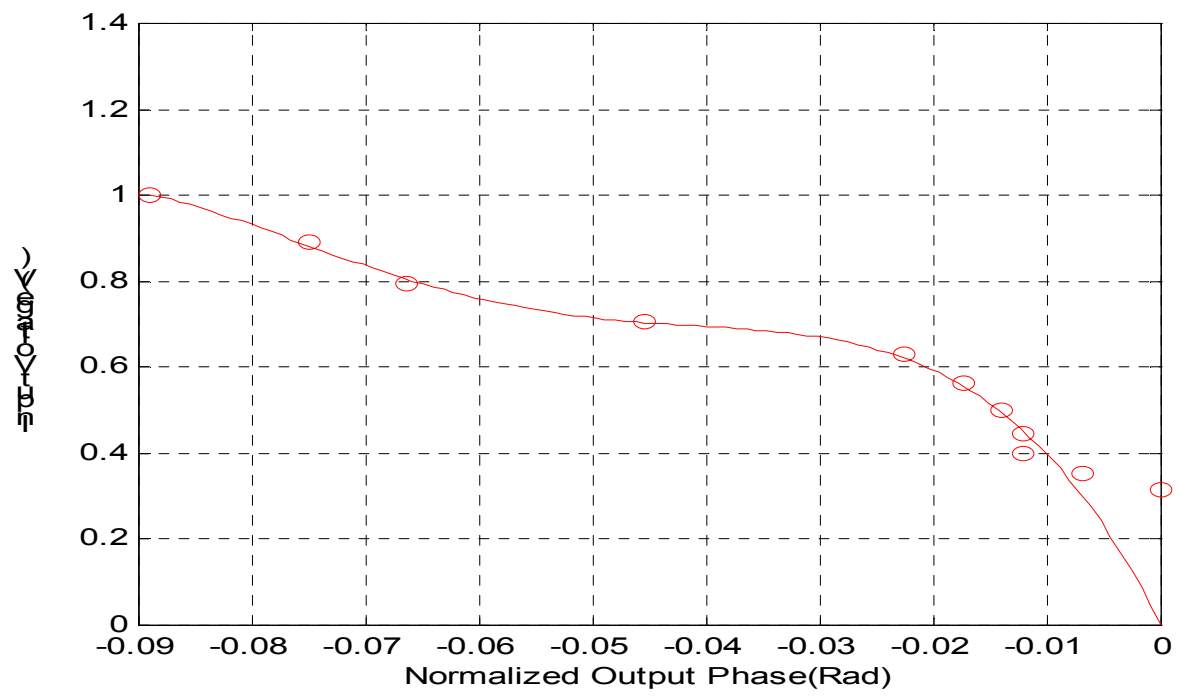

Figure 2.11 Curve Fitting of Inverse of Normalized Phase Saturation Curve ( $5^{\text {th }}$ order curve fitting) 
Table 2.8 Klystron Parameters

\begin{tabular}{|c|c|c|c|}
\hline $\bar{c}_{1}$ & -7.6080 & $\bar{d}_{1}$ & 0.5096 \\
\hline $\bar{c}_{2}$ & 67.6050 & $\bar{d}_{2}$ & 0.2742 \\
\hline $\bar{c}_{3}$ & -220.4201 & $\bar{d}_{3}$ & -25.5832 \\
\hline $\bar{c}_{4}$ & 391.2620 & $\bar{d}_{4}$ & 107.9442 \\
\hline $\bar{c}_{5}$ & -392.2802 & $\bar{d}_{5}$ & -188.2361 \\
\hline $\bar{c}_{6}$ & 207.0155 & $\bar{d}_{6}$ & 150.5679 \\
\hline $\bar{c}_{7}$ & -44.5728 & $\bar{d}_{7}$ & -45.5657 \\
\hline
\end{tabular}

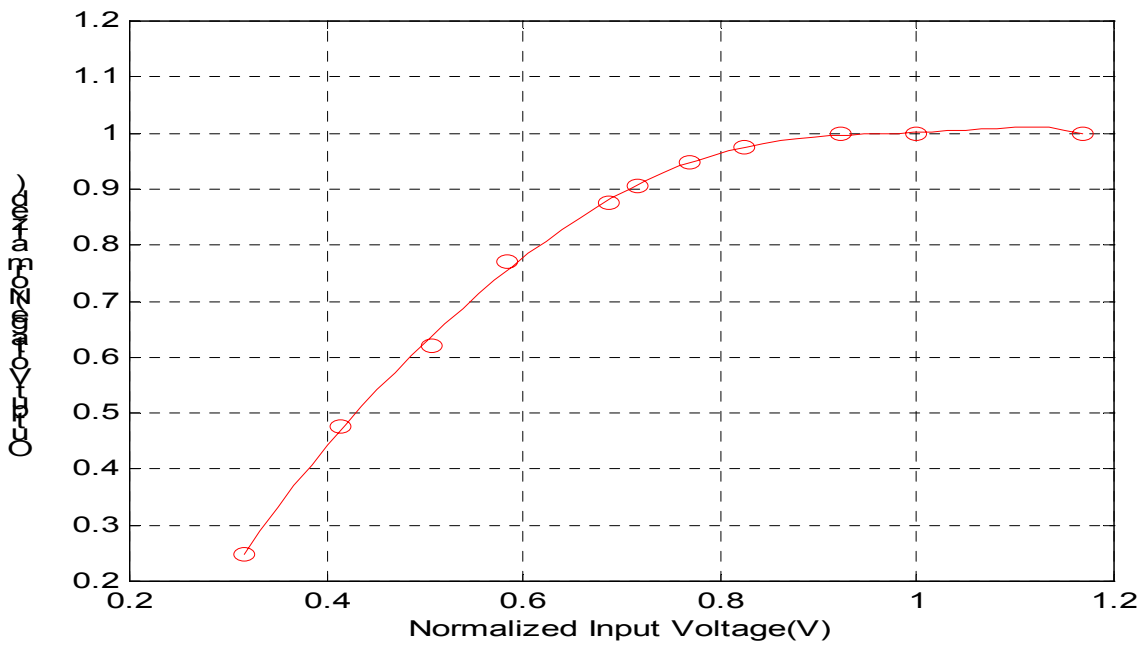

Figure 2.12 Curve Fitting of Normalized Amplitude Saturation Curve ( $7^{\text {th }}$ order curve fitting)

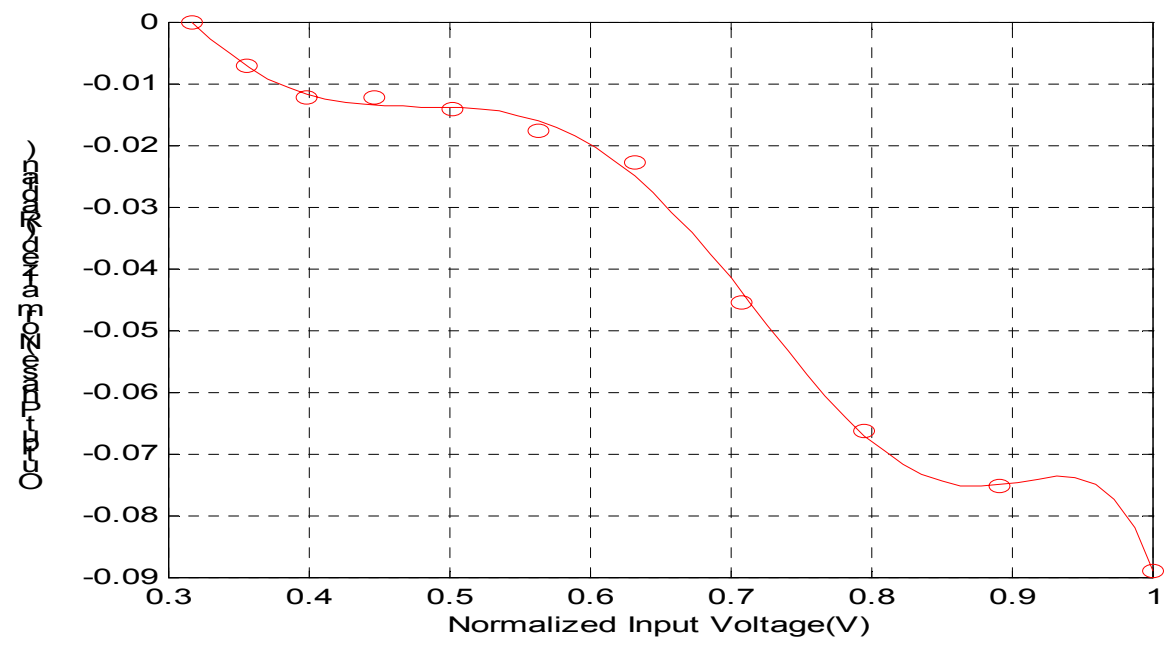

Figure 2.13 Curve Fitting of Normalized Phase Saturation Curve ( $7^{\text {th }}$ order curve fitting) 
Table 2.9 Klystron Parameters

\begin{tabular}{|c|c|c|c|}
\hline $\bar{e}_{1}$ & $0.0227 \mathrm{e}+003$ & $\bar{f}_{1}$ & -90.3879 \\
\hline $\bar{e}_{2}$ & $-0.2384 \mathrm{e}+003$ & $\bar{f}_{2}$ & $-9.0582 \mathrm{e}+003$ \\
\hline $\bar{e}_{3}$ & $1.0481 \mathrm{e}+003$ & $\bar{f}_{3}$ & $-5.6029 \mathrm{e}+005$ \\
\hline $\bar{e}_{4}$ & $-2.3786 \mathrm{e}+003$ & $\bar{f}_{4}$ & $-1.8088 \mathrm{e}+007$ \\
\hline $\bar{e}_{5}$ & $2.9435 \mathrm{e}+003$ & $\bar{f}_{5}$ & $-3.0254 \mathrm{e}+008$ \\
\hline $\bar{e}_{6}$ & $-1.8888 \mathrm{e}+003$ & $\bar{f}_{6}$ & $-2.4973 \mathrm{e}+009$ \\
\hline $\bar{e}_{7}$ & $0.4926 \mathrm{e}+003$ & $\bar{f}_{7}$ & $-8.0556 \mathrm{e}+009$ \\
\hline
\end{tabular}

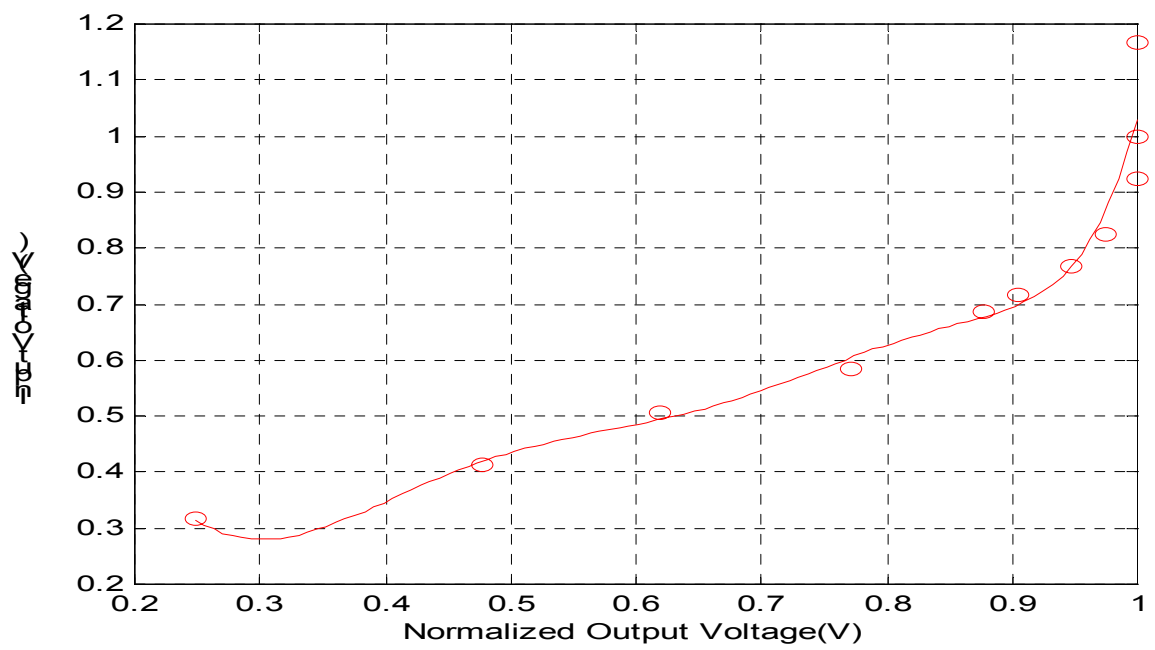

Figure 2.14 Curve Fitting of Inverse of Normalized Amplitude Saturation Curve ( $7^{\text {th }}$ order curve fitting)

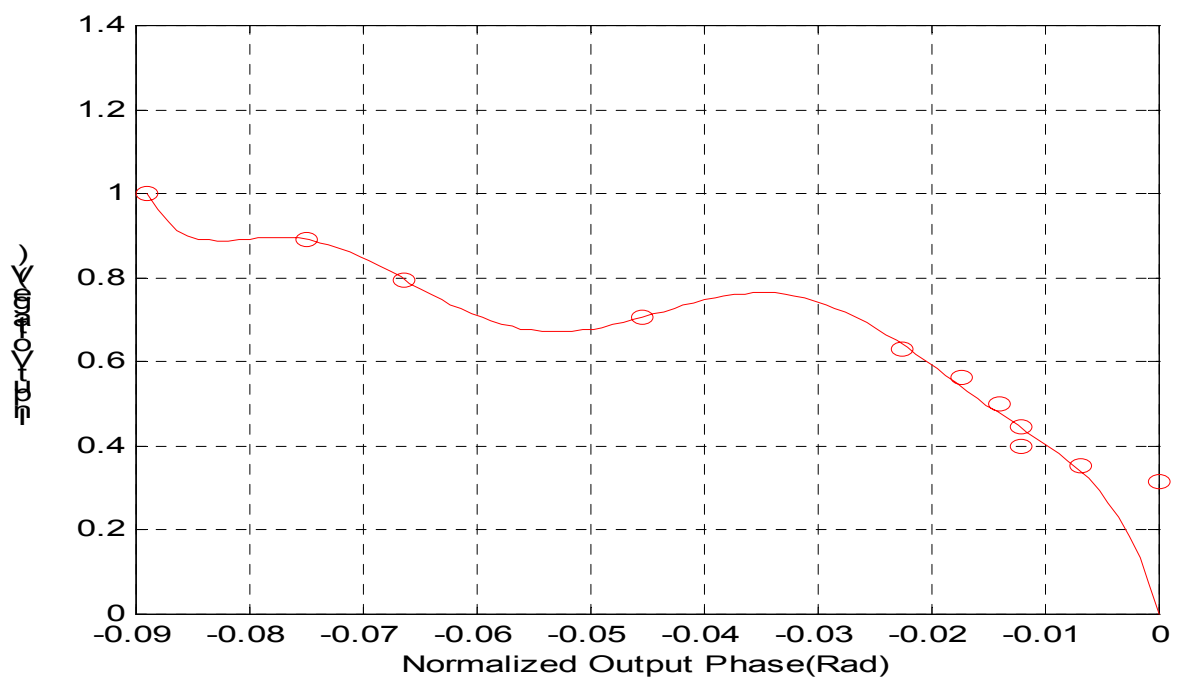

Figure 2.15 Curve Fitting of Inverse of Normalized Phase Saturation Curve ( $7^{\text {th }}$ order curve fitting) 


\section{II-C. STATE SPACE MODEL OF A KLYSTRON}

As mentioned, a klystron can be expressed as the cascade of the linear subsystem and the nonlinear output subsystem. The linear subsystem represents the $3 \mathrm{~dB}$ bandwidth of the klystron and the constant gain. The nonlinear output subsystem represents the amplitude saturation curve and the phase saturation curve of the klystron.

\section{II-C-1. Nonlinear Model}

The klystron dynamics are mainly determined by the $3 \mathrm{~dB}$ bandwidth, $f_{3 d B}$, of it and are simply represented by

$$
\begin{aligned}
& \dot{x}_{2}=-a x_{1}+a u_{1} \\
& \dot{x}_{2}=-a x_{2}+a u_{2}
\end{aligned}
$$

where $u_{1}$ and $u_{2}$ are low level RF In-phase (I) and Quadrature (Q) signals and $a=2 \pi f_{3 d B}$.

It is assumed that the signal levels of the low level RF I and Q are nominally less than or equal to one. This assumption is important for the analysis and synthesis of the control system. When the signal levels are much higher than one, a scale matrix is introduced. There is a preamplifier in order to level up the low level RF I and Q and there is a gain of the klystron at a specific operating point of the klystron. A constant $K_{g}$ is defined as the lumped gain of the preamplifier gain and the klystron gain. As shown in table 2.2, the klystron gain is different from one operating point to another operating point. Hence, the input voltage $V_{\text {in }}$ of the klystron is

$$
V_{i n}=K_{g} \sqrt{x_{1}^{2}+x_{2}^{2}}
$$

The normalized input voltage $V_{i n}^{N}$ as mentioned in the previous section is

$$
V_{i n}^{N}=\frac{V_{i n}}{V_{i n}^{\text {Sat }}}=\frac{K_{g}}{\sqrt{2 R_{o} P_{i n}^{\text {Sat }}}} \sqrt{x_{1}^{2}+x_{2}^{2}}
$$

For notational convenience, let

$$
A=V_{\text {in }}^{N}
$$

Considering the analytic equations for the Amplitude saturation curve and the Phase saturation curve as investigated in the previous section, the normalized output voltage, $V_{\text {out }}^{N}$ and the normalized output phase, $\theta_{\text {out }}^{N}$ of the klystron at baseband are modeled as

$$
\begin{aligned}
& V_{\text {out }}^{N}=I_{A}(A)=\sum_{i=1}^{N} \bar{c}_{i} A^{i} \\
& \theta_{\text {out }}^{N}=I_{P}(A)+\bar{\phi}=\sum_{i=1}^{N} \bar{d}_{i} A^{i}+\bar{\phi}
\end{aligned}
$$


respectively, where $\bar{\phi}$ is the filtered phase of the input signals (low level RF I and Q signals). That is,

$$
\bar{\phi}=\tan ^{-1}\left(\frac{x_{2}}{x_{1}}\right) .
$$

The equations (2.15)-(2.16) and (2.20)-(2.21) define the nonlinear state space model of the normalized klystron.

The output voltage as given in (2-20) is the normalized one, $V_{\text {out }}^{N}$. Hence, the unnormalized output voltage for driving the cavity is obtained from the equation (2.4).

$$
V_{\text {out }}=V_{\text {out }}^{\text {Sat }} \cdot V_{\text {out }}^{N}=\sqrt{2 R_{o} P_{\text {out }}^{\text {Sat }}} \cdot V_{\text {out }}^{N} \text {. }
$$

Plugging (2.23) into (2.20), the unnormalized output voltage and the unnormalized output phase of the klystron are obtained.

$$
\begin{aligned}
& V_{\text {out }}=\sqrt{2 R_{o} P_{\text {out }}^{\text {Sat }}} \cdot V_{\text {out }}^{N}=\sqrt{2 R_{o} P_{\text {out }}^{\text {Sat }}} \cdot I_{A}(A)=\sqrt{2 R_{o} P_{\text {out }}^{\text {Sat }}} \cdot \sum_{i=1}^{N} \bar{c}_{i} A^{i} \\
& \theta_{\text {out }}=I_{P}(A)+\bar{\phi}=\sum_{i=1}^{N} \bar{d}_{i} A^{i}+\bar{\phi} .
\end{aligned}
$$

The output amplitude and the output phase as given in (2.24)-(2.25) can be expressed in the form of Inphase (I) and Quadrature (Q),

$$
\begin{aligned}
I_{y} & =V_{\text {out }} \cos \left(\theta_{\text {out }}\right)=\sqrt{2 R_{o} P_{\text {out }}^{\text {Sat }}} \cdot \sum_{i=1}^{N} \bar{c}_{i} A^{i} \cos \left(\sum_{i=1}^{N} \bar{d}_{i} A^{i}+\bar{\phi}\right) \\
& =\sqrt{2 R_{o} P_{\text {out }}^{\text {Sat }}} \cdot \sum_{i=1}^{N} \bar{c}_{i} A^{i}\left(\cos \left(\sum_{i=1}^{N} \bar{d}_{i} A^{i}\right) \cos (\bar{\phi})-\sin \left(\sum_{i=1}^{N} \bar{d}_{i} A^{i}\right) \sin (\bar{\phi})\right) \\
Q_{y} & =V_{\text {out }} \sin \left(\theta_{\text {out }}\right)=\sqrt{2 R_{o} P_{\text {out }}^{\text {Sat }}} \cdot \sum_{i=1}^{N} \bar{c}_{i} A^{i} \sin \left(\sum_{i=1}^{N} \bar{d}_{i} A^{i}+\bar{\phi}\right) \\
& =\sqrt{2 R_{o} P_{\text {out }}^{\text {Sat }}} \cdot \sum_{i=1}^{N} \bar{c}_{i} A^{i}\left(\sin \left(\sum_{i=1}^{N} \bar{d}_{i} A^{i}\right) \cos (\bar{\phi})+\cos \left(\sum_{i=1}^{N} \bar{d}_{i} A^{i}\right) \sin (\bar{\phi})\right) .
\end{aligned}
$$

The equations (2-15)-(2.16) and (2-26)-(2.27) define the nonlinear state space model of the unnormalized klystron.

\section{II-C-2. Linear Parameter Varying Model}

The nonlinear model of a klystron given by (2-15)-(2.16) and (2-26)-(2.27) depicts the nonlinear amplitude saturation curve and the nonlinear phase saturation curve of a klystron. However, the nonlinearity hinders the application of the modern linear control theory both for analysis and synthesis. As mentioned, the linear accelerator in SNS requires 92 klystrons. Also, each klystron is operated at a different operating power due to the different energy level to be developed at each portion of the linear accelerator. In order to achieve efficient analyses and syntheses for these klystrons and further for the cascades of klystrons and cavities 
in the linear accelerator, a linear klystron model around each operating point is required where the operating point is determined by the required power of a cavity.

(2.26) and (2.27) are compactly expressed as

$$
\left[\begin{array}{l}
I_{y} \\
Q_{y}
\end{array}\right]=\sqrt{2 R_{o} P_{\text {out }}^{\text {Sat }}} \cdot \sum_{i=1}^{N} \bar{c}_{i} A^{i}\left[\begin{array}{cc}
\cos \left(\sum_{i=1}^{N} \bar{d}_{i} A^{i}\right) & -\sin \left(\sum_{i=1}^{N} \bar{d}_{i} A^{i}\right) \\
\sin \left(\sum_{i=1}^{N} \bar{d}_{i} A^{i}\right) & \cos \left(\sum_{i=1}^{N} \bar{d}_{i} A^{i}\right)
\end{array}\right]\left[\begin{array}{c}
\cos (\bar{\phi}) \\
\sin (\bar{\phi})
\end{array}\right] .
$$

Define

$$
\begin{aligned}
& K_{A}(A)=\sqrt{\frac{P_{\text {out }}^{\text {Sat }}}{P_{\text {in }}^{\text {Sat }}}} \cdot\left(\sum_{i=1}^{N} \bar{c}_{i} A^{i-1}\right) \\
& K_{P}(A)=\sum_{i=1}^{N} \bar{d}_{i} A^{i-1}
\end{aligned}
$$

Plugging (2.18) and (2.19) into (2.28) yields

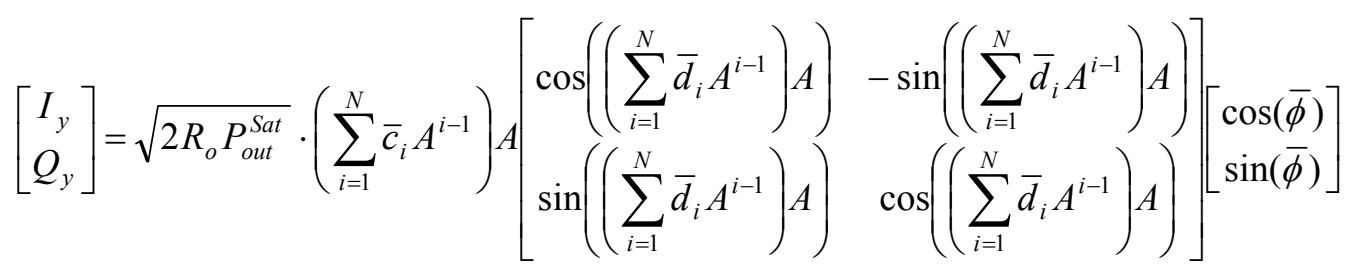

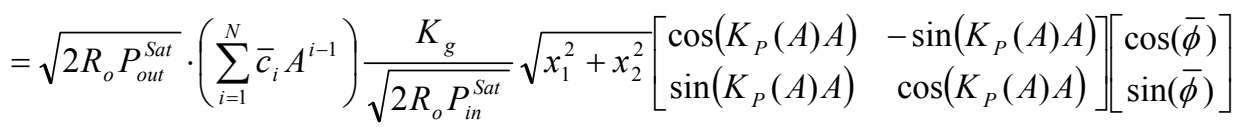

$$
\begin{aligned}
& =K_{g} \frac{\sqrt{2 R_{o} P_{\text {out }}^{\text {Sat }}}}{\sqrt{2 R_{o} P_{\text {in }}^{\text {Sat }}}} \cdot\left(\sum_{i=1}^{N} \bar{c}_{i} A^{i-1}\right)\left[\begin{array}{rr}
\cos \left(K_{P}(A) A\right) & -\sin \left(K_{P}(A) A\right) \\
\sin \left(K_{P}(A) A\right) & \cos \left(K_{P}(A) A\right)
\end{array}\right] \sqrt{x_{1}^{2}+x_{2}^{2}}\left[\begin{array}{c}
\cos (\bar{\phi}) \\
\sin (\bar{\phi})
\end{array}\right] \\
& =K_{g} \sqrt{\frac{P_{\text {out }}^{\text {Sat }}}{P_{\text {in }}^{\text {Sat }}}} \cdot\left(\sum_{i=1}^{N} \bar{c}_{i} A^{i-1}\right)\left[\begin{array}{cc}
\cos \left(K_{P}(A) A\right) & -\sin \left(K_{P}(A) A\right) \\
\sin \left(K_{P}(A) A\right) & \cos \left(K_{P}(A) A\right)
\end{array}\right] \sqrt{x_{1}^{2}+x_{2}^{2}}\left[\begin{array}{c}
\cos (\bar{\phi}) \\
\sin (\bar{\phi})
\end{array}\right] \\
& =K_{g} K_{A}(A)\left[\begin{array}{cc}
\cos \left(K_{P}(A) A\right) & -\sin \left(K_{P}(A) A\right) \\
\sin \left(K_{P}(A) A\right) & \cos \left(K_{P}(A) A\right)
\end{array}\right] \sqrt{x_{1}^{2}+x_{2}^{2}}\left[\begin{array}{c}
\cos (\bar{\phi}) \\
\sin (\bar{\phi})
\end{array}\right]
\end{aligned}
$$

Since

$$
\left[\begin{array}{l}
x_{1} \\
x_{2}
\end{array}\right]=\sqrt{x_{1}^{2}+x_{2}^{2}}\left[\begin{array}{l}
\cos (\bar{\phi}) \\
\sin (\bar{\phi})
\end{array}\right]
$$

equation (2.31) is reduced to

$$
\left[\begin{array}{l}
I_{y} \\
Q_{y}
\end{array}\right]=K_{g} K_{A}(A)\left[\begin{array}{cc}
\cos \left(K_{P}(A) A\right) & -\sin \left(K_{P}(A) A\right) \\
\sin \left(K_{P}(A) A\right) & \cos \left(K_{P}(A) A\right)
\end{array}\right]\left[\begin{array}{l}
x_{1} \\
x_{2}
\end{array}\right] .
$$


Since the gain $K_{g}$ is a function of the normalized input voltage $A$ of the amplitude saturation and phase saturation curves, the I-Q output equation (2.32) is a linear parameter varying equation. That is, if $\mathrm{x}, \mathrm{u}, \mathrm{y}$, $A_{k}, B_{k}, C_{k}(A)$ are defined by

$$
\begin{aligned}
& x=\left[\begin{array}{ll}
x_{1} & x_{2}
\end{array}\right]^{T}, \quad u=\left[\begin{array}{ll}
u_{1} & u_{2}
\end{array}\right]^{T}, \quad y=\left[\begin{array}{ll}
I_{y} & Q_{y}
\end{array}\right]^{T} \\
& A_{k}=\left[\begin{array}{cc}
-a & 0 \\
0 & -a
\end{array}\right], \\
& B_{k}=\left[\begin{array}{ll}
a & 0 \\
0 & a
\end{array}\right], \\
& C_{k}(A)=K_{g} K_{A}(A)\left[\begin{array}{cc}
\cos \left(K_{P}(A) A\right) & -\sin \left(K_{P}(A) A\right) \\
\sin \left(K_{P}(A) A\right) & \cos \left(K_{P}(A) A\right)
\end{array}\right],
\end{aligned}
$$

then the klystron model as given in (2.15)-(2.16) and (2.32) is given by

$$
\begin{aligned}
& \dot{x}=A_{k} x+B_{k} u \\
& y=C_{k}(A) x .
\end{aligned}
$$

Note that from (2.18)-(2.19), $A$ is expressed in terms of $x_{1}, x_{2}$. Hence, $C_{k}(A)$ can be expressed as the $x_{1}, x_{2}$ dependent matrix. However, the operating point of the klystron is determined based on the RF power for the cavity rather than the input low level RF signals, which implies that it is more reasonable to consider the signal $A$ first and then to consider the signals $x_{1}, x_{2}$. As a consequence, as given in (2.33), expressing the matrix $C_{k}$ in terms of $A$ is much more consistent.

\section{II-C-3. Linear Hybrid Model}

The linear parameter varying klystron model as given in (2.34) and (2.35) can catch the transient behaviors in the period of cavity filling and in the period of beam loading. In order for that to be possible, it is necessary to continuously measure or estimate the trajectory of the point $\left(V_{\text {out }}, \theta_{\text {out }}\right)$, which is a difficult task. Instead, the operating point $A_{d}$ of $A$ is considered and the model is written by

$$
\begin{aligned}
& \dot{x}=A_{k} x+B_{k} u \\
& y=C_{k}\left(A_{d}\right) x,
\end{aligned}
$$

where $A_{d}$ is obtained as follows.

Let $\left(V_{\text {out }}^{d}, \theta_{\text {out }}^{d}\right)$ be the desired operating output of the unnormalized outputs (2.24) and (2.25). $\left(V_{\text {out }}^{d}, \theta_{\text {out }}^{d}\right)$ can be obtained from the operating condition of a given cavity. The desired operating output of the normalized klystron is

$$
\begin{aligned}
& V_{\text {out }}^{N d}=\frac{1}{\sqrt{2 R_{o} P_{\text {out }}^{\text {Sat }}}} \cdot V_{\text {out }}^{d}, \\
& \theta_{\text {out }}^{N d}=\theta_{\text {out }}^{d} .
\end{aligned}
$$

By the inverse of the amplitude saturation curve (2.13), the desired operating input voltage $A_{d}$ for the normalized amplitude saturation curve is obtained. 


$$
A_{d}=I_{A}^{-1}\left(V_{\text {out }}^{N d}\right)=\sum_{i=1}^{N} \bar{e}_{i}\left(V_{\text {out }}^{N d}\right)^{i}
$$

Also, the desired operating voltage of the low level RF signal is obtained from (2.18).

$$
\sqrt{x_{1}^{2}+x_{2}^{2}}=\frac{\sqrt{2 R_{o} P_{i n}^{S a t}}}{K_{g}} A_{d}
$$

From (2.21), the desired operating phase of the low level RF signal is obtained.

$$
\bar{\phi}_{d}=\theta_{\text {out }}^{N d}-\sum_{i=1}^{N} \bar{d}_{i} A_{d}^{i}=\theta_{\text {out }}^{N d}-\sum_{i=1}^{N} \bar{d}_{i}\left(\sum_{j=1}^{N} \bar{e}_{j}\left(V_{\text {out }}^{N d}\right)^{j}\right)^{i}
$$

Hence, from the relations given in (2.18) and (2.22), the desired operating I and Q of the low level RF system are

$$
\begin{aligned}
& x_{1 d}=\frac{\sqrt{2 R_{o} P_{i n}^{\text {Sat }}}}{K_{g}} A_{d} \cos \left(\bar{\phi}_{d}\right) \\
& x_{2 d}=\frac{\sqrt{2 R_{o} P_{\text {in }}^{\text {Sat }}}}{K_{g}} A_{d} \sin \left(\bar{\phi}_{d}\right) .
\end{aligned}
$$

\section{II-C-4. Lyapunov Linearization Model}

The Lyapunov linearization is the most popular modeling method for the nonlinear system where the input variation, state variation, and the output variation are described as $u_{\delta}=u-u_{d}, x_{\delta}=x-x_{d}, y_{\delta}=y-y_{d}$, respectively, and the linear model is expressed as

$$
\begin{aligned}
& \dot{x}_{\delta}=A_{k} x_{\delta}+B_{k} u_{\delta} \\
& y_{\delta}=C_{k}\left(A_{d}\right) x_{\delta} .
\end{aligned}
$$

\section{II-C-5. Comparison of Linear Models}

The linear parameter varying model given by (2.34)-(2.35) depicts the nonlinearities of the klystron dynamics very well, even though in order to obtain the amplitude $A$, the trajectory of $\left(V_{\text {out }}, \theta_{\text {out }}\right)$ is necessary, which results in complexity. It also covers the whole operating range of the klystron. The model as given in (2.36)-(2.37) describes the klystron dynamics around the wider neighborhood of the operating point $A_{d}$ than the Lyapunov linearization model of (2.26)-(2.27). The relations between the linear parameter varying model (2.34)-(2.35) and the linear time invariant model (2.36)-(2.37) are shown in figures 2.16-2.19. Figure 2.16 and figure 2.17 show the output responses of the nonlinear klystron model, the linear parameter varying model (2.34)-(2.35), and the linear model (2.36)-(2.37). For the inputs $u_{1}$ and $u_{2}$ that reflect the cavity filling time for the normal conducting cavity in SNS linear accelerator, different transient dynamics are observed. The transient behavior of the linear model (2.36)-(2.37) is far different from that of the nonlinear model. This is due to the output matrix $C_{k}$ which is obtained from the linearization at the operating point $A_{d}$. However, as the input $A$ to the amplitude saturation curve and the 
phase saturation curve approaches the operating point $A_{d}$, the outputs of the linear model (2.36)- (2.37) converge to the outputs of the nonlinear model. Similar phenomena are observed for the linear parameter varying model (2.34)-(2.35) but in this case, the convergent region is wider than that of the linear model (2.36)-(2.37). These phenomena explain the convergent region as shown in figure 2.18. In figure 2.18, the convergent region for the Lyapunov linearization is illustrated for comparison.

Comparing with the linear model (2.36)-(2.37), in the Lyapunov linearization, instead of $u, x, y$ in the model (2.36)-(2.37), $u_{\delta}, x_{\delta}, y_{\delta}$ are used for the model.

Figure 2.19 shows the signal relations at the subblocks of the klystron model which clarify the relations of the two models (2-34)-(2.35) and (2.36)-(2.37).

In our modeling of the low level RF control system, the linear hybrid model is used for the controller synthesis and closed loop system analysis. For the verification of synthesis and analysis, the nonlinear model is implemented with MATLAB/SIMULINK blocks.

In SNS, the Linac part is composed of 1 RFQ, 6 DTL tanks, 4 CCL modules, and 81 SRF cavities. A 2.5 MW klystron supplies RF power to a DTL TANK, a 5.0 MW klystron supplies RF power to a CCL module, and a $550 \mathrm{~kW}$ klystron supplies RF power to a SRF cavity. The energy to be developed at each cavity is different from cavity to cavity, which implies that the operating point of each klystron is different from klystron to klystron. Table 2.8 shows the power and voltage relations at 6 DTL tanks. Figure 2.20 shows the frequency responses of the 6 klystrons for 6 DTK tanks. The transfer matrices are obtained based on the state space model (2.36)-(2.37). Figure 2.21 and figure 2.22 show the amplitude sturation curves for the 6 klystrons. The amplitude saturation curves are unnormalized for the klystron saturation voltages, $V_{\text {out }}^{\text {Sat }}$ 's as given in the $9^{\text {th }}$ column of table 2.8. The operating output voltages are $V_{\text {out }}^{\text {OP }}$ 's as given in the $6^{\text {th }}$ column of table 2.8. Note that the normalized operating input voltages $A_{d}$ 's are the same value, which implies that with the same input $u$, the gains, $K_{g}$ 's, are determined so that

$$
\frac{K_{g 1}}{\sqrt{2 R_{o} P_{i n}^{\text {Sat } 1}}}=\frac{K_{g 2}}{\sqrt{2 R_{o} P_{i n}^{\text {Sat } 2}}}=\cdots=\frac{K_{g 6}}{\sqrt{2 R_{o} P_{i n 6}^{\text {Sat }}}} .
$$

Table 2.8 SNS DTL Tank RF Power Requirement (Transmission Line Characteristic Impedance is $50 \Omega$ )

\begin{tabular}{|c|c|c|c|c|c|c|c|c|}
\hline Tank & $\begin{array}{c}\mathrm{P}_{\mathrm{B}} \\
(\mathrm{MW})\end{array}$ & $\begin{array}{c}\mathrm{P}_{\mathrm{C}} \\
(\mathrm{MW})\end{array}$ & $\begin{array}{c}\mathrm{P}_{\mathrm{f}} \\
(\mathrm{MW})\end{array}$ & $\begin{array}{c}\text { Klystron } \\
\text { Operating } \\
\text { Point Power } \\
(\mathrm{MW})\end{array}$ & $\begin{array}{c}\text { Klystron } \\
\text { Operating } \\
\text { Point Voltage } \\
(\mathrm{kV})\end{array}$ & $\begin{array}{c}\text { Power } \\
\text { Control } \\
\text { Margin }(\%)\end{array}$ & $\begin{array}{c}\text { Klystron } \\
\text { Saturation } \\
\text { Power } \\
(\mathrm{MW})\end{array}$ & $\begin{array}{c}\text { Klystron } \\
\text { Saturation } \\
\text { Voltage } \\
(\mathrm{kV})\end{array}$ \\
\hline 1 & 0.171 & 0.337 & 0.508 & 0.508 & 7.1274 & 25 & 0.6350 & 7.9687 \\
\hline 2 & 0.521 & 1.046 & 1.567 & 1.567 & 12.5180 & 25 & 1.9588 & 13.9955 \\
\hline 3 & 0.576 & 1.302 & 1.878 & 1.878 & 13.7040 & 25 & 2.3475 & 15.3216 \\
\hline 4 & 0.571 & 1.259 & 1.830 & 1.830 & 13.5277 & 25 & 2.2875 & 15.1245 \\
\hline 5 & 0.571 & 1.270 & 1.841 & 1.841 & 13.5683 & 25 & 2.3013 & 15.1699 \\
\hline 6 & 0.487 & 1.281 & 1.768 & 1.768 & 13.2966 & 25 & 2.2100 & 14.8661 \\
\hline
\end{tabular}




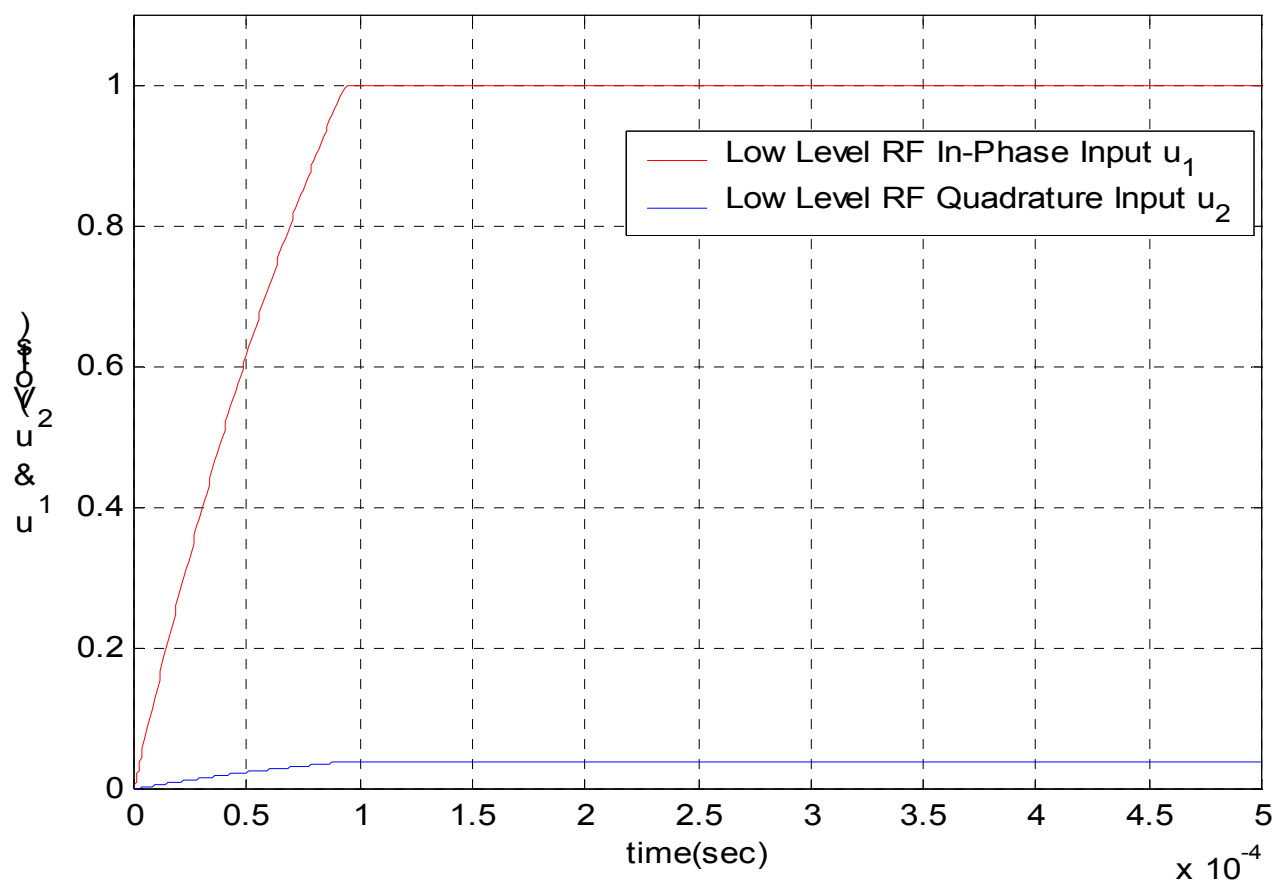

Figure 2.16 Inputs $u_{1}$ and $u_{2}$ that reflect the cavity filling time for the normal conducting cavity 

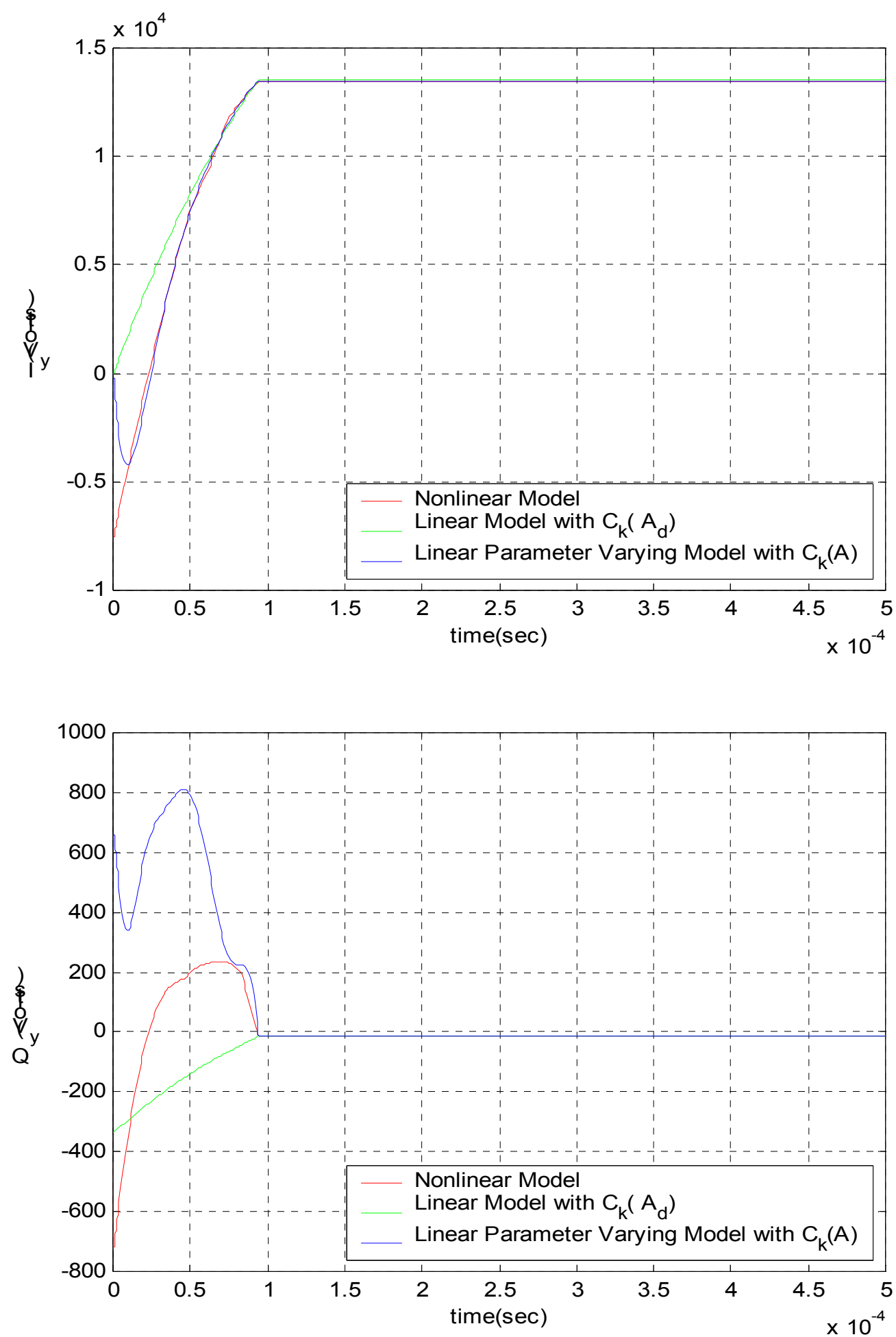

Figure 2.17 Output Responses of the nonlinear model, the linear parameter varying model (equations (2.34)-(2.35)), and the linear model (equations (2.36)-(2.37)) 


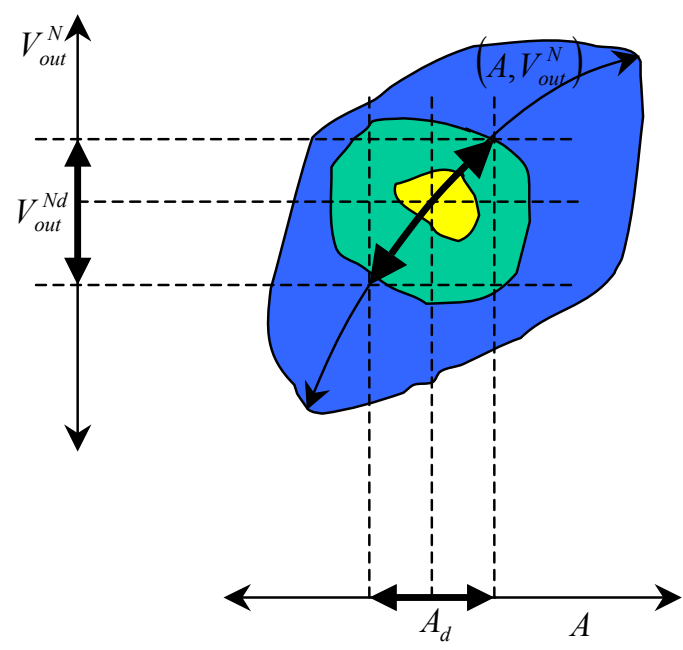

Yellow Region: Lyapunov Linearization

Green Region: Linear Hybrid Model

Blue Region: Linear Parameter Varying Model

Figure 2.18 Operating Point and Convergent Regions

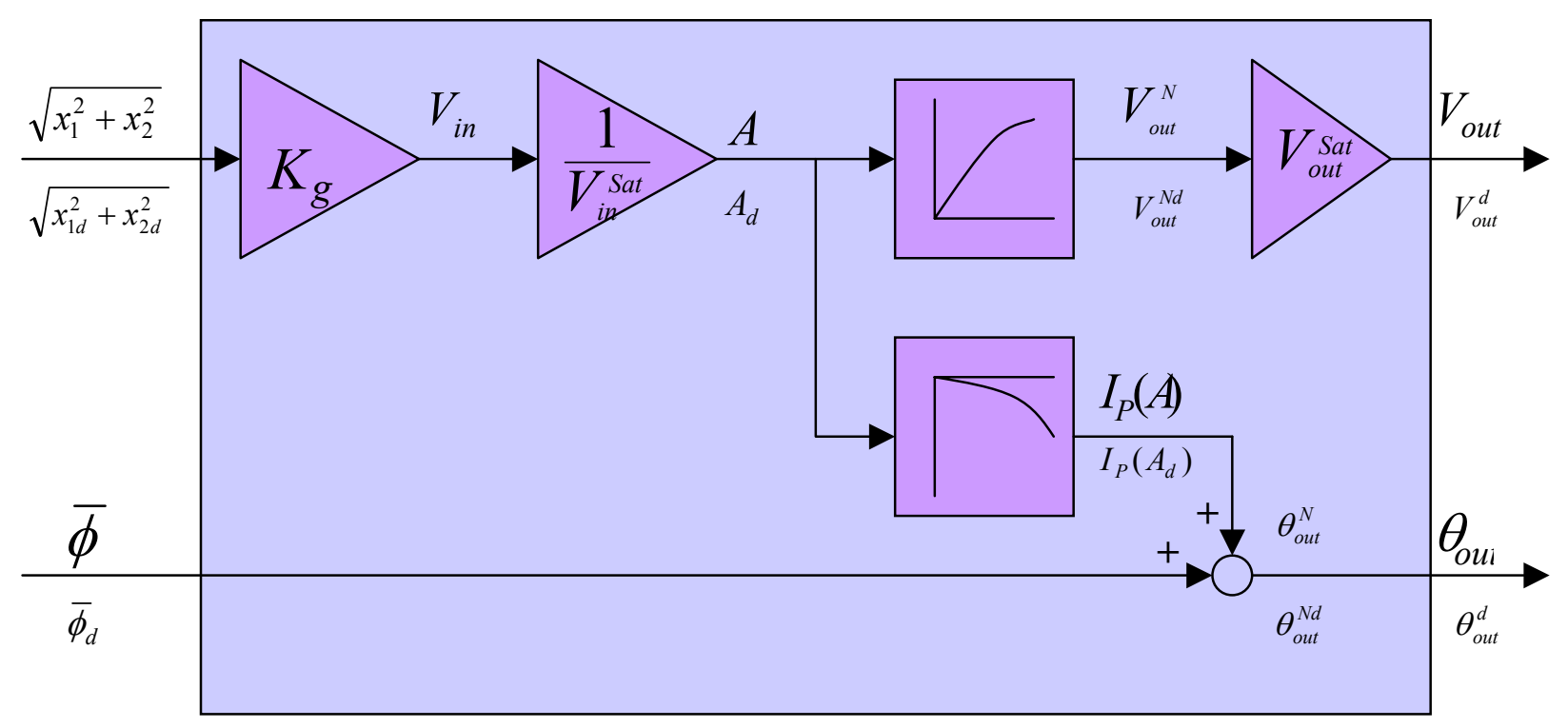

Figure 2.19 Block Diagram of a Klystron 

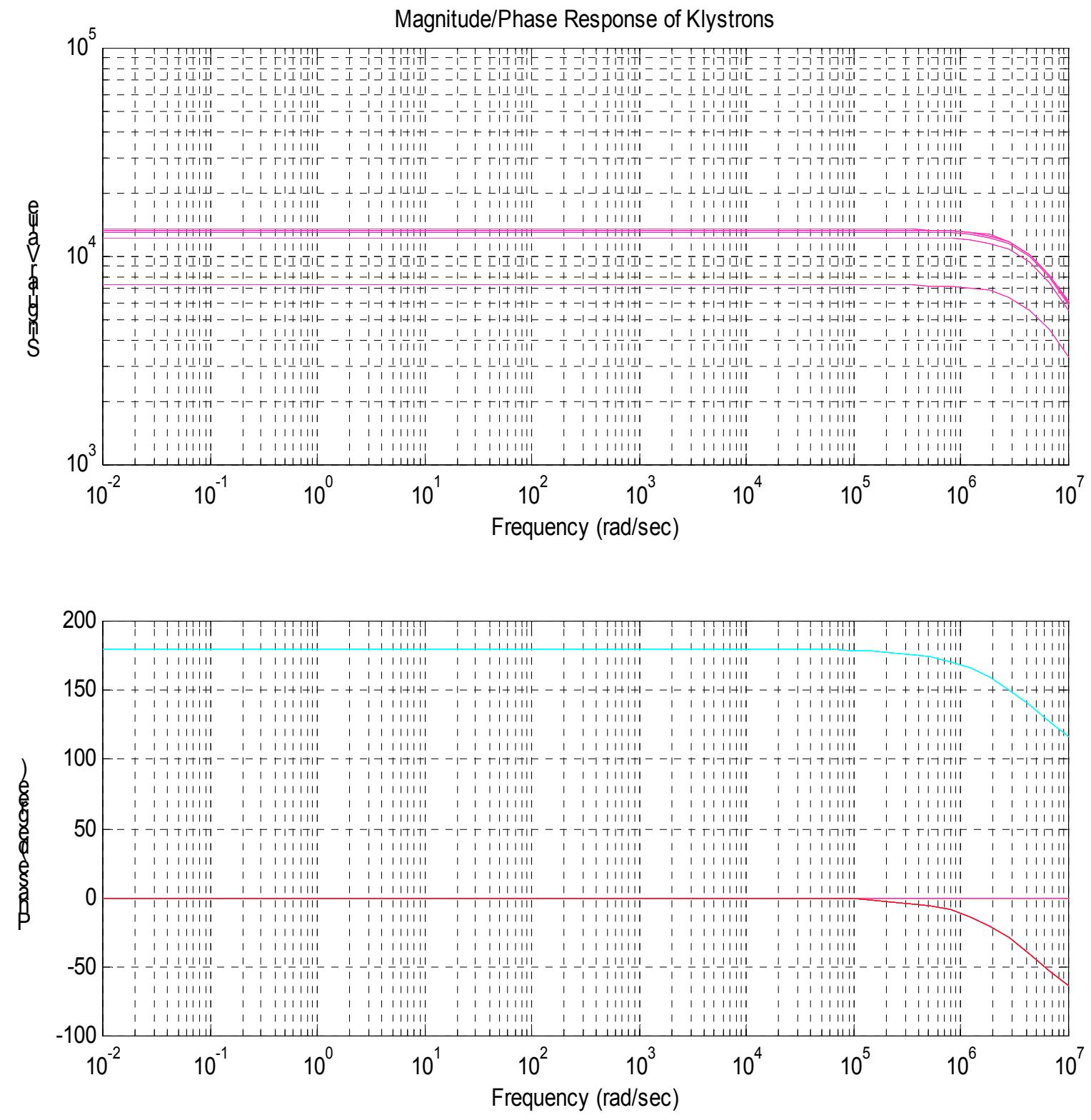

Figure 2.20 Frequency Responses of 6 Klystrons Driving 6 DTL Tanks. Note that the klystron is modeled as two input two output (TITO) system. Each klystron has different magnitude response. The phase response plot for a klystron has 4 curves ( 1 curve is folded with the magenta curve). The phase responses of 6 klystrons are identical. 


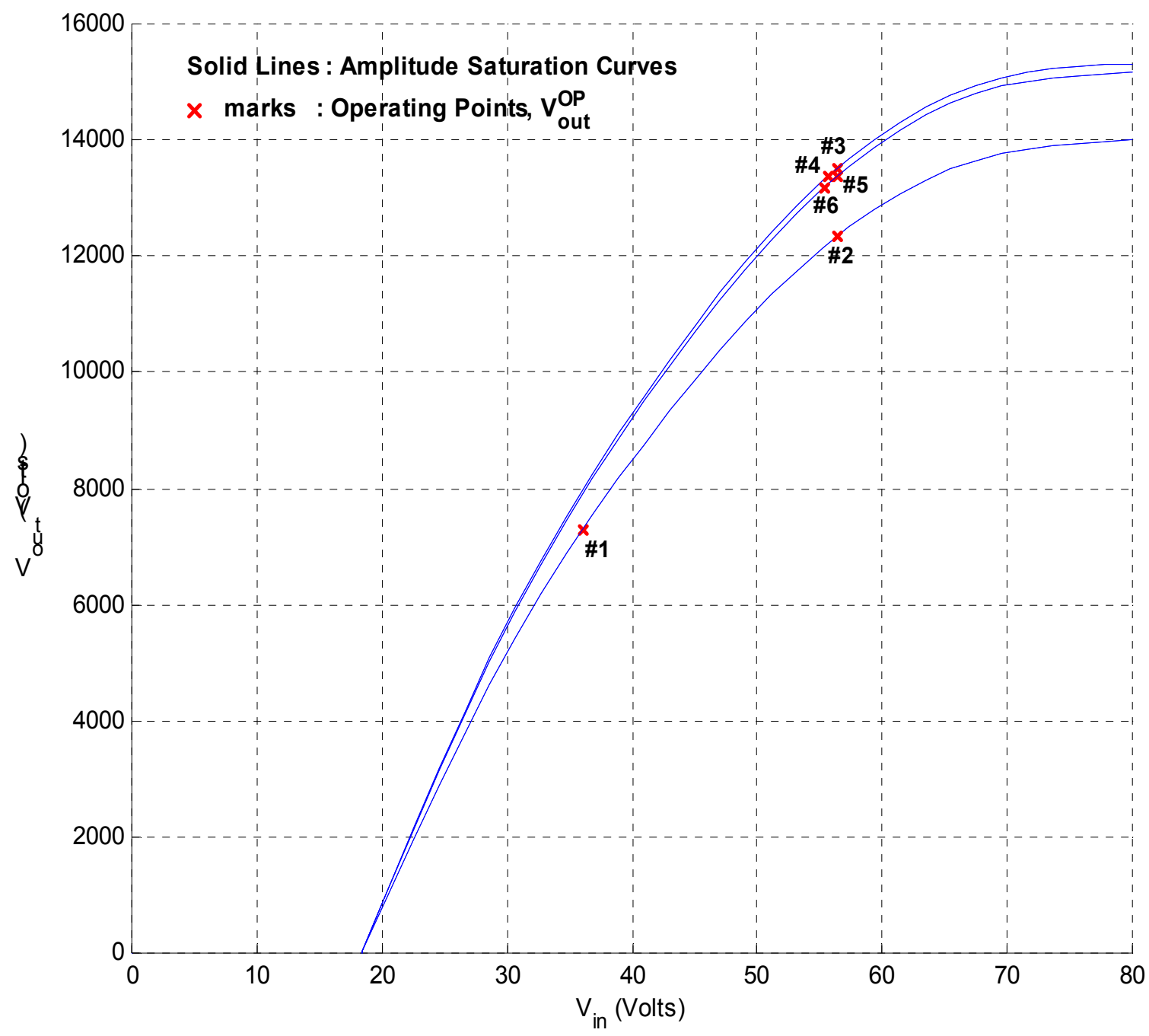

Figure 2.21 Amplitude Saturation Curves of 6 Klystrons Driving the 6 DTL Tanks 


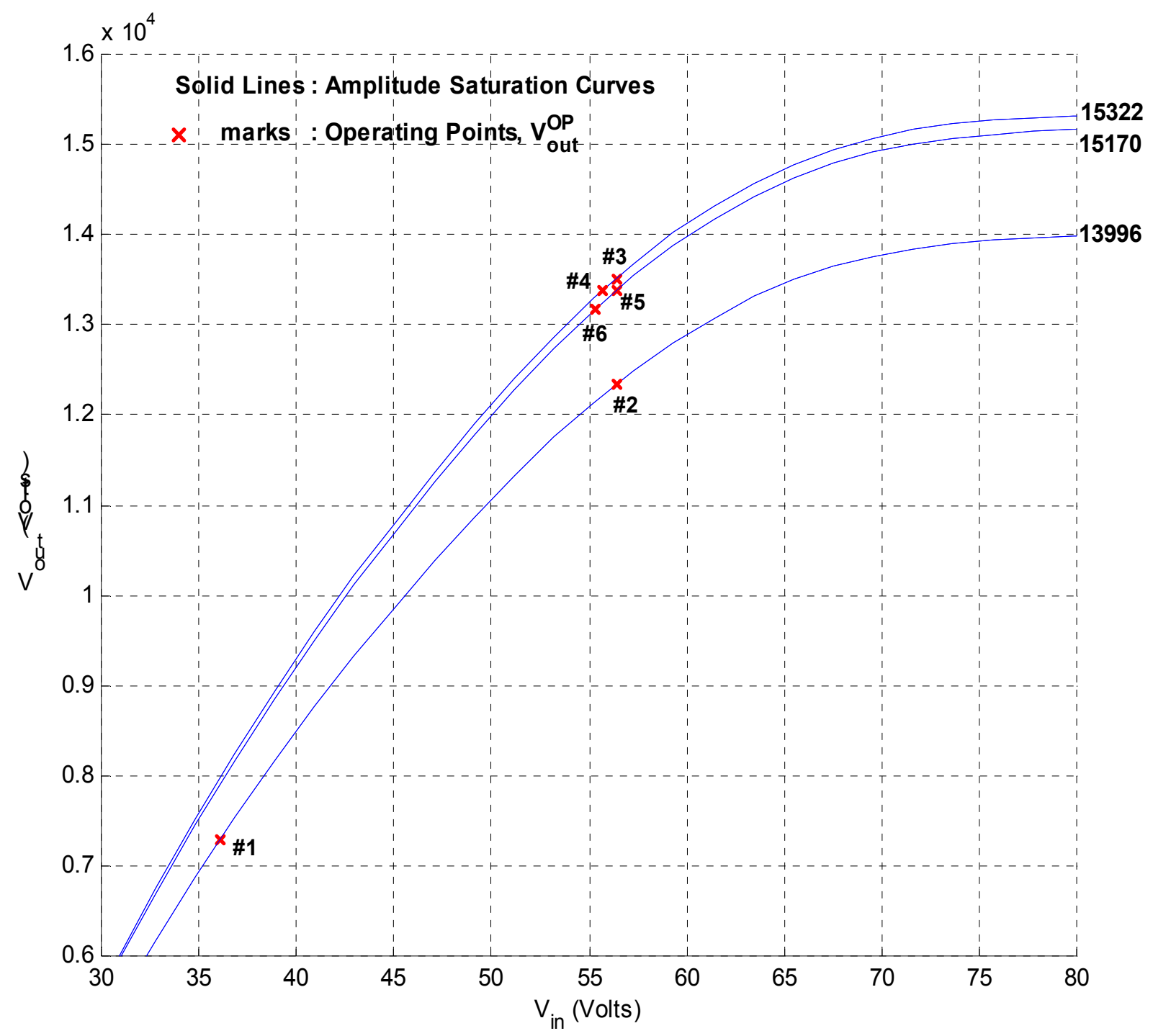

Figure 2.22 Close-up of Amplitude Saturation Curves of 6 Klystrons Driving the 6 DTL Tanks 


\section{II-D. PERTURBATION MODEL OF A KLYSTRON}

The major perturbation of a klystron's output is due to the high voltage power supply (HVPS) ripple. HVPS ripple changes both the amplitude and the phase of the klystron output, which results in perturbation of both In-phase and Quadrature outputs of the klystron. A perturbed klystron due to HVPS ripple can be represented by a nominal system with multiplicative uncertainty or additive uncertainty. Multiplicative uncertainty and additive uncertainty are interchangable. Another way is to express a perturbed klystron as a nominal system with exogenous disturbance. In the latter case, a proper transfer matrix from the HVPS ripple to the klystron output should be obtained.

Let $A$ be the normalized nominal input voltage of the amplitude saturation curve. Then the perturbed input voltage due to the HVPS ripple is expressed as

$$
A_{p}=A\left(1+\Delta_{A} R\right)^{1.25}
$$

where $\Delta_{A}$ is the amplitude perturbation in percentage and $R \in \mathfrak{R},|R| \leq 1$, is the normalized ripple signal.

Usually, the perturbation $\Delta_{A}$ is small. Hence, (2.43) can be approximated by

$$
A_{p} \approx A\left(1+1.25 \Delta_{A} R\right)
$$

Also,

$$
\left(1+1.25 \Delta_{A} R\right)^{i} \approx 1+1.25 \cdot i \cdot \Delta_{A} R
$$

Then, from (2.11), the perturbed normalized output voltage of the klystron is obtained.

$$
\begin{aligned}
y_{1 P}^{N} & =I_{A}\left(A_{P}\right)=\sum_{i=1}^{N} \bar{c}_{i} A_{P}^{i} \\
& =\sum_{i=1}^{N} \bar{c}_{i}\left(A\left(1+1.25 \Delta_{A} R\right)\right)^{i} \\
& =\sum_{i=1}^{N} \bar{c}_{i} A^{i}\left(1+1.25 \Delta_{A} R\right)^{i} \\
& \approx \sum_{i=1}^{N} \bar{c}_{i} A^{i}\left(1+1.25 \cdot i \cdot \Delta_{A} R\right) \\
& =\sum_{i=1}^{N} \bar{c}_{i} A^{i}+1.25 \Delta_{A} R \sum_{i=1}^{N} \bar{c}_{i} A^{i} \cdot i .
\end{aligned}
$$

In order to simplify the second term of (2.46), a constant $\hat{k}_{A}$ of the following optimization problem is to be obtained. 


$$
\begin{array}{cc}
\min & k \\
\text { s.t. } \quad & \sum_{i=1}^{N} \bar{c}_{i} A^{i} \cdot i-k \sum_{i=1}^{N} \bar{c}_{i} A^{i} \leq 0
\end{array}
$$

The solution of the linear programming problem (2.47) always exists. If the solution of the linear programming problem (2.47) is $\hat{k}_{A}$, then (2.46) can be expressed as

$$
y_{1 P}^{N}=\sum_{i=1}^{N} \bar{c}_{i} A^{i}+1.25 \hat{k}_{A} \Delta_{A} R \sum_{i=1}^{N} \bar{c}_{i} A^{i}
$$

Hence, the perturbed normalized output voltage of the klystron due to the HVPS ripple is written by

$$
y_{1 P}^{N}=\sum_{i=1}^{N} \bar{c}_{i} A^{i}\left(1+1.25 \hat{k}_{A} \Delta_{A} R\right)
$$

The effect of HVPS ripple on the output phase of the klystron is described by two terms. The first is the perturbed output of the phase saturation curve given in (2.12) due to the perturbation of the perturbed normalized input voltage $A_{P}$,

$$
\sum_{i=1}^{N} \bar{d}_{i} A_{P}^{i}
$$

and the second is the direct additive phase perturbation

$$
\Delta_{P} R .
$$

Taking into consideration these two terms, the perturbed normalized output phase of the klystron is expressed as

$$
\begin{aligned}
y_{2 P}^{N} & =\sum_{i=1}^{N} \bar{d}_{i} A_{P}^{i}+\Delta_{P} R+\bar{\phi} \\
& =\sum_{i=1}^{N} \bar{d}_{i}\left(A\left(1+1.25 \Delta_{A} R\right)\right)^{i}+\Delta_{P} R+\bar{\phi} \\
& =\sum_{i=1}^{N} \bar{d}_{i} A^{i}\left(1+1.25 \Delta_{A} R\right)^{i}+\Delta_{P} R+\bar{\phi} \\
& \approx \sum_{i=1}^{N} \bar{d}_{i} A^{i}\left(1+1.25 \cdot i \cdot \Delta_{A} R\right)+\Delta_{P} R+\bar{\phi} \\
& =\sum_{i=1}^{N} \bar{d}_{i} A^{i}+1.25 \Delta_{A} R \sum_{i=1}^{N} \bar{d}_{i} A^{i} \cdot i+\Delta_{P} R+\bar{\phi} .
\end{aligned}
$$

In order to simplify (2.50), a constant $\hat{k}_{P}$ of the solution of the following optimization problem is to be obtained. 


$$
\begin{array}{cc}
\min & k \\
\text { s.t. } & \sum_{i=1}^{N} \bar{d}_{i} A^{i} \cdot i-k \sum_{i=1}^{N} \bar{d}_{i} A^{i} \leq 0
\end{array}
$$

By using the solution $\hat{k}_{P}$ of the optimization problem (2.51), the perturbed normalized output phase of the klystron due to the HVPS ripple can be expressed as

$$
\begin{aligned}
y_{2 P}^{N} & =\sum_{i=1}^{N} \bar{d}_{i} A^{i}+1.25 \hat{k}_{P} \Delta_{A} R \sum_{i=1}^{N} \bar{d}_{i} A^{i}+\Delta_{P} R+\bar{\phi} \\
& =\sum_{i=1}^{N} \bar{d}_{i} A^{i}\left(1+1.25 \hat{k}_{P} \Delta_{A} R\right)+\Delta_{P} R+\bar{\phi} .
\end{aligned}
$$

Also, from (2.49) and (2.52), the perturbed unnormalized output voltage and the perturbed output phase of the klystron can be obtained.

$$
\begin{aligned}
& y_{1 p}=\sqrt{2 R_{o} P_{\text {out }}^{\text {Sat }}} \cdot \sum_{i=1}^{N} \bar{c}_{i} A^{i}\left(1+1.25 \hat{k}_{A} \Delta_{A} R\right) \\
& y_{2 p}=\sum_{i=1}^{N} \bar{d}_{i} A^{i}\left(1+1.25 \hat{k}_{P} \Delta_{A} R\right)+\Delta_{P} R+\bar{\phi} .
\end{aligned}
$$

In the In-phase and Quadrature coordinates, the perturbed unnormalized output of the klystron is expressed as

$$
\begin{aligned}
& I_{y P}= y_{1 P} \cos \left(y_{2 P}\right)=\sqrt{2 R_{o} P_{\text {out }}^{\text {Sat }}} \cdot \sum_{i=1}^{N} \bar{c}_{i} A^{i}\left(1+1.25 \hat{k}_{A} \Delta_{A} R\right) \cos \left(\sum_{i=1}^{N} \bar{d}_{i} A^{i}\left(1+1.25 \hat{k}_{P} \Delta_{A} R\right)+\Delta_{P} R+\bar{\phi}\right) \\
&=\sqrt{2 R_{o} P_{\text {out }}^{\text {Sat }}} \cdot\left(1+1.25 \hat{k}_{A} \Delta_{A} R\right) \sum_{i=1}^{N} \bar{c}_{i} A^{i}\left(\cos \left(\sum_{i=1}^{N} \bar{d}_{i} A^{i}\left(1+1.25 \hat{k}_{P} \Delta_{A} R\right)+\Delta_{P} R\right) \cos (\bar{\phi})\right. \\
&\left.-\sin \left(\sum_{i=1}^{N} \bar{d}_{i} A^{i}\left(1+1.25 \hat{k}_{P} \Delta_{A} R\right)+\Delta_{P} R\right) \sin (\bar{\phi})\right) \\
& \begin{aligned}
& Q_{y P}=y_{1 P} \sin \left(y_{2 P}\right)=\sqrt{2 R_{o} P_{\text {out }}^{\text {Sat }}} \cdot \sum_{i=1}^{N} \bar{c}_{i} A^{i}\left(1+1.25 \hat{k}_{A} \Delta_{A} R\right) \sin \left(\sum_{i=1}^{N} \bar{d}_{i} A^{i}\left(1+1.25 \hat{k}_{P} \Delta_{A} R\right)+\Delta_{P} R+\bar{\phi}\right) \\
&=\sqrt{2 R_{o} P_{\text {out }}^{\text {Sat }}} \cdot\left(1+1.25 \hat{k}_{A} \Delta_{A} R\right)_{i=1}^{N} \bar{c}_{i} A^{i}\left(\sin \left(\sum_{i=1}^{N} \bar{d}_{i} A^{i}\left(1+1.25 \hat{k}_{P} \Delta_{A} R\right)+\Delta_{P} R\right) \cos (\bar{\phi})\right. \\
&\left.+\cos \left(\sum_{i=1}^{N} \bar{d}_{i} A^{i}\left(1+1.25 \hat{k}_{P} \Delta_{A} R\right)+\Delta_{P} R\right) \sin (\bar{\phi})\right) .
\end{aligned}
\end{aligned}
$$

The above equations are compactly expressed in matrix form as

$$
\left[\begin{array}{c}
I_{y P} \\
Q_{y P}
\end{array}\right]=\sqrt{2 R_{o} P_{\text {out }}^{\text {Sat }}} \cdot\left(1+1.25 \hat{k}_{A} \Delta_{A} R\right) \sum_{i=1}^{N} \bar{c}_{i} A^{i}
$$




$$
\cdot\left[\begin{array}{rr}
\cos \left(\sum_{i=1}^{N} \bar{d}_{i} A^{i}\left(1+1.25 \hat{k}_{P} \Delta_{A} R\right)+\Delta_{P} R\right) & -\sin \left(\sum_{i=1}^{N} \bar{d}_{i} A^{i}\left(1+1.25 \hat{k}_{P} \Delta_{A} R\right)+\Delta_{P} R\right) \\
\sin \left(\sum_{i=1}^{N} \bar{d}_{i} A^{i}\left(1+1.25 \hat{k}_{P} \Delta_{A} R\right)+\Delta_{P} R\right) & \cos \left(\sum_{i=1}^{N} \bar{d}_{i} A^{i}\left(1+1.25 \hat{k}_{P} \Delta_{A} R\right)+\Delta_{P} R\right)
\end{array}\right]\left[\begin{array}{c}
\cos (\bar{\phi}) \\
\sin (\bar{\phi})
\end{array}\right]
$$

Plugging (2.18) and (2.19) into (2.55) and using (2.29) and (2.30) yields

$$
\begin{aligned}
& {\left[\begin{array}{l}
I_{y} \\
Q_{y}
\end{array}\right]=\sqrt{2 R_{o} P_{\text {out }}^{\text {Sat }}} \cdot\left(1+1.25 \hat{k}_{A} \Delta_{A} R\right) \cdot\left(\sum_{i=1}^{N} \bar{c}_{i} A^{i-1}\right) A} \\
& \cdot\left[\begin{array}{lr}
\cos \left(K_{P}(A) A\left(1+1.25 \hat{k}_{P} \Delta_{A} R\right)+\Delta_{P} R\right) & -\sin \left(K_{P}(A) A\left(1+1.25 \hat{k}_{P} \Delta_{A} R\right)+\Delta_{P} R\right) \\
\sin \left(K_{P}(A) A\left(1+1.25 \hat{k}_{P} \Delta_{A} R\right)+\Delta_{P} R\right) & \cos \left(K_{P}(A) A\left(1+1.25 \hat{k}_{P} \Delta_{A} R\right)+\Delta_{P} R\right)
\end{array}\right]\left[\begin{array}{c}
\cos (\bar{\phi}) \\
\sin (\bar{\phi})
\end{array}\right] \\
& =\sqrt{2 R_{o} P_{\text {out }}^{\text {Sat }}} \cdot\left(1+1.25 \hat{k}_{A} \Delta_{A} R\right) \cdot\left(\sum_{i=1}^{N} \bar{c}_{i} A^{i-1}\right) \frac{K_{g}}{\sqrt{2 R_{o} P_{i n}^{\text {Sat }}}} \sqrt{x_{1}^{2}+x_{2}^{2}} \\
& \cdot\left[\begin{array}{cc}
\cos \left(K_{P}(A) A\left(1+1.25 \hat{k}_{P} \Delta_{A} R\right)+\Delta_{P} R\right) & -\sin \left(K_{P}(A) A\left(1+1.25 \hat{k}_{P} \Delta_{A} R\right)+\Delta_{P} R\right) \\
\sin \left(K_{P}(A) A\left(1+1.25 \hat{k}_{P} \Delta_{A} R\right)+\Delta_{P} R\right) & \cos \left(K_{P}(A) A\left(1+1.25 \hat{k}_{P} \Delta_{A} R\right)+\Delta_{P} R\right)
\end{array}\right]\left[\begin{array}{c}
\cos (\bar{\phi}) \\
\sin (\bar{\phi})
\end{array}\right] \\
& =K_{g} \frac{\sqrt{2 R_{o} P_{\text {out }}^{\text {Sat }}}}{\sqrt{2 R_{o} P_{\text {in }}^{\text {Sat }}}} \cdot\left(1+1.25 \hat{k}_{A} \Delta_{A} R\right) \cdot\left(\sum_{i=1}^{N} \bar{c}_{i} A^{i-1}\right) \\
& \cdot\left[\begin{array}{cc}
\cos \left(K_{P}(A) A\left(1+1.25 \hat{k}_{P} \Delta_{A} R\right)+\Delta_{P} R\right) & -\sin \left(K_{P}(A) A\left(1+1.25 \hat{k}_{P} \Delta_{A} R\right)+\Delta_{P} R\right) \\
\sin \left(K_{P}(A) A\left(1+1.25 \hat{k}_{P} \Delta_{A} R\right)+\Delta_{P} R\right) & \cos \left(K_{P}(A) A\left(1+1.25 \hat{k}_{P} \Delta_{A} R\right)+\Delta_{P} R\right)
\end{array}\right] \sqrt{x_{1}^{2}+x_{2}^{2}}\left[\begin{array}{c}
\cos (\bar{\phi}) \\
\sin (\bar{\phi})
\end{array}\right] \\
& =K_{g} \sqrt{\frac{P_{\text {out }}^{\text {Sat }}}{P_{\text {in }}^{\text {Sat }}}} \cdot\left(1+1.25 \hat{k}_{A} \Delta_{A} R\right) \cdot\left(\sum_{i=1}^{N} \bar{c}_{i} A^{i-1}\right) \\
& \cdot\left[\begin{array}{cc}
\cos \left(K_{P}(A) A\left(1+1.25 \hat{k}_{P} \Delta_{A} R\right)+\Delta_{P} R\right) & -\sin \left(K_{P}(A) A\left(1+1.25 \hat{k}_{P} \Delta_{A} R\right)+\Delta_{P} R\right) \\
\sin \left(K_{P}(A) A\left(1+1.25 \hat{k}_{P} \Delta_{A} R\right)+\Delta_{P} R\right) & \cos \left(K_{P}(A) A\left(1+1.25 \hat{k}_{P} \Delta_{A} R\right)+\Delta_{P} R\right)
\end{array}\right] \sqrt{x_{1}^{2}+x_{2}^{2}}\left[\begin{array}{c}
\cos (\bar{\phi}) \\
\sin (\bar{\phi})
\end{array}\right]
\end{aligned}
$$

Since

$$
\left[\begin{array}{l}
x_{1} \\
x_{2}
\end{array}\right]=\sqrt{x_{1}^{2}+x_{2}^{2}}\left[\begin{array}{c}
\cos (\bar{\phi}) \\
\sin (\bar{\phi})
\end{array}\right],
$$

and since

$$
\begin{aligned}
& {\left[\begin{array}{lr}
\cos \left(K_{P}(A) A\left(1+1.25 \hat{k}_{P} \Delta_{A} R\right)+\Delta_{P} R\right) & -\sin \left(K_{P}(A) A\left(1+1.25 \hat{k}_{P} \Delta_{A} R\right)+\Delta_{P} R\right) \\
\sin \left(K_{P}(A) A\left(1+1.25 \hat{k}_{P} \Delta_{A} R\right)+\Delta_{P} R\right) & \cos \left(K_{P}(A) A\left(1+1.25 \hat{k}_{P} \Delta_{A} R\right)+\Delta_{P} R\right)
\end{array}\right]} \\
& =\left[\begin{array}{lr}
\cos \left(K_{P}(A) A \cdot 1.25 \hat{k}_{P} \Delta_{A} R+\Delta_{P} R\right) & -\sin \left(K_{P}(A) A \cdot 1.25 \hat{k}_{P} \Delta_{A} R+\Delta_{P} R\right) \\
\sin \left(K_{P}(A) A \cdot 1.25 \hat{k}_{P} \Delta_{A} R+\Delta_{P} R\right) & \cos \left(K_{P}(A) A \cdot 1.25 \hat{k}_{P} \Delta_{A} R+\Delta_{P} R\right)
\end{array}\right]\left[\begin{array}{cc}
\cos \left(K_{P}(A) A\right) & -\sin \left(K_{P}(A) A\right) \\
\sin \left(K_{P}(A) A\right) & \cos \left(K_{P}(A) A\right)
\end{array}\right]
\end{aligned}
$$

Equation (2.56) can be written as 


$$
\begin{gathered}
{\left[\begin{array}{l}
I_{y} \\
Q_{y}
\end{array}\right]=\left(1+1.25 \hat{k}_{A} \Delta_{A} R\right)\left[\begin{array}{rr}
\cos \left(K_{P}(A) A \cdot 1.25 \hat{k}_{P} \Delta_{A} R+\Delta_{P} R\right) & -\sin \left(K_{P}(A) A \cdot 1.25 \hat{k}_{P} \Delta_{A} R+\Delta_{P} R\right) \\
\sin \left(K_{P}(A) A \cdot 1.25 \hat{k}_{P} \Delta_{A} R+\Delta_{P} R\right) & \cos \left(K_{P}(A) A \cdot 1.25 \hat{k}_{P} \Delta_{A} R+\Delta_{P} R\right)
\end{array}\right]} \\
\cdot K_{g} K_{A}(A)\left[\begin{array}{rr}
\cos \left(K_{P}(A) A\right) & -\sin \left(K_{P}(A) A\right) \\
\sin \left(K_{P}(A) A\right) & \cos \left(K_{P}(A) A\right)
\end{array}\right]\left[\begin{array}{c}
x_{1} \\
x_{2}
\end{array}\right]
\end{gathered}
$$

As in the previous section, define $\mathrm{x}, \mathrm{u}, \mathrm{y}, A_{k}, B_{k}, C_{k}(A)$ by

$$
\begin{aligned}
& x=\left[\begin{array}{ll}
x_{1} & x_{2}
\end{array}\right]^{T}, \quad u=\left[\begin{array}{ll}
u_{1} & u_{2}
\end{array}\right]^{T}, \quad y=\left[\begin{array}{ll}
I_{y} & Q_{y}
\end{array}\right]^{T} \\
& A_{k}=\left[\begin{array}{cc}
-a & 0 \\
0 & -a
\end{array}\right], \quad B_{k}=\left[\begin{array}{cc}
a & 0 \\
0 & a
\end{array}\right], \\
& C_{k}(A)=K_{g} K_{A}(A)\left[\begin{array}{cc}
\cos \left(K_{P}(A) A\right) & -\sin \left(K_{P}(A) A\right) \\
\sin \left(K_{P}(A) A\right) & \cos \left(K_{P}(A) A\right)
\end{array}\right],
\end{aligned}
$$

and define the perturbation matrix $C_{\Delta}\left(A, \Delta_{A}, R\right)$ by

$$
C_{\Delta}\left(A, \Delta_{A}, R\right)=\left(1+1.25 \hat{k}_{A} \Delta_{A} R\right)\left[\begin{array}{rr}
\cos \left(K_{P}(A) A \cdot 1.25 \hat{k}_{P} \Delta_{A} R+\Delta_{P} R\right) & -\sin \left(K_{P}(A) A \cdot 1.25 \hat{k}_{P} \Delta_{A} R+\Delta_{P} R\right) \\
\sin \left(K_{P}(A) A \cdot 1.25 \hat{k}_{P} \Delta_{A} R+\Delta_{P} R\right) & \cos \left(K_{P}(A) A \cdot 1.25 \hat{k}_{P} \Delta_{A} R+\Delta_{P} R\right)
\end{array}\right] .
$$

Then the perturbed klystron model is defined by (2.15)-(2.16) and (2.58).

$$
\begin{aligned}
& \dot{x}=A_{k} x+B_{k} u \\
& y=C_{\Delta}\left(A, \Delta_{A}, R\right) C_{k}(A) x .
\end{aligned}
$$

The effect of the HVPS on the output of the klystron is seen in two ways. The first is the amplitude perturbation which is given by

$$
\left(1+1.25 \hat{k}_{A} \Delta_{A} R\right)
$$

The second is the phase perturbation $K_{P}(A) A \cdot 1.25 \hat{k}_{P} \Delta_{A} R+\Delta_{P} R$ which is given by the perturbed rotation matrix

$$
\left[\begin{array}{rr}
\cos \left(K_{P}(A) A \cdot 1.25 \hat{k}_{P} \Delta_{A} R+\Delta_{P} R\right) & -\sin \left(K_{P}(A) A \cdot 1.25 \hat{k}_{P} \Delta_{A} R+\Delta_{P} R\right) \\
\sin \left(K_{P}(A) A \cdot 1.25 \hat{k}_{P} \Delta_{A} R+\Delta_{P} R\right) & \cos \left(K_{P}(A) A \cdot 1.25 \hat{k}_{P} \Delta_{A} R+\Delta_{P} R\right)
\end{array}\right] .
$$

Now, the effect of HVPS on the klystron is to be described as the multiplicative uncertainty.

First, by Taylor series expansion, (2.62) is approximated as 


$$
\begin{aligned}
& {\left[\begin{array}{cc}
\cos \left(K_{P}(A) A \cdot 1.25 \hat{k}_{P} \Delta_{A} R+\Delta_{P} R\right) & -\sin \left(K_{P}(A) A \cdot 1.25 \hat{k}_{P} \Delta_{A} R+\Delta_{P} R\right) \\
\sin \left(K_{P}(A) A \cdot 1.25 \hat{k}_{P} \Delta_{A} R+\Delta_{P} R\right) & \cos \left(K_{P}(A) A \cdot 1.25 \hat{k}_{P} \Delta_{A} R+\Delta_{P} R\right)
\end{array}\right]} \\
& \approx\left[\begin{array}{cc}
1-\frac{1}{2}\left(K_{P}(A) A \cdot 1.25 \hat{k}_{P} \Delta_{A}+\Delta_{P}\right)^{2} R^{2} & -\left(K_{P}(A) A \cdot 1.25 \hat{k}_{P} \Delta_{A}+\Delta_{P}\right) R \\
\left(K_{P}(A) A \cdot 1.25 \hat{k}_{P} \Delta_{A}+\Delta_{P}\right) R & 1-\frac{1}{2}\left(K_{P}(A) A \cdot 1.25 \hat{k}_{P} \Delta_{A}+\Delta_{P}\right)^{2} R^{2}
\end{array}\right]
\end{aligned}
$$

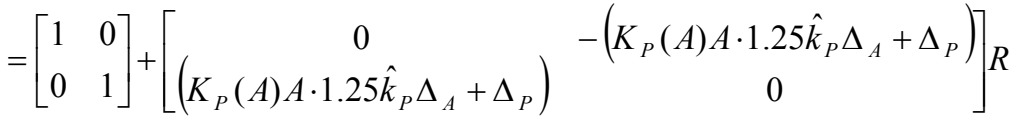

$$
\begin{aligned}
& +\left[\begin{array}{cc}
-\frac{1}{2}\left(K_{P}(A) A \cdot 1.25 \hat{k}_{P} \Delta_{A}+\Delta_{P}\right)^{2} & 0 \\
0 & -\frac{1}{2}\left(K_{P}(A) A \cdot 1.25 \hat{k}_{P} \Delta_{A}+\Delta_{P}\right)^{2}
\end{array}\right] R^{2}
\end{aligned}
$$

As a result, the perturbation matrix (2.58) is expressed as

$$
\begin{aligned}
C_{\Delta}\left(A, \Delta_{A}, R\right) \approx\left[\begin{array}{ll}
1 & 0 \\
0 & 1
\end{array}\right]+\left[\begin{array}{cc}
1.25 \hat{k}_{A} \Delta_{A} & -\left(K_{P}(A) A \cdot 1.25 \hat{k}_{P} \Delta_{A}+\Delta_{P}\right) \\
\left(K_{P}(A) A \cdot 1.25 \hat{k}_{P} \Delta_{A}+\Delta_{P}\right) & 1.25 \hat{k}_{A} \Delta_{A}
\end{array}\right] R \\
+\left[\begin{array}{cc}
-\frac{1}{2}\left(K_{P}(A) A \cdot 1.25 \hat{k}_{P} \Delta_{A}+\Delta_{P}\right)^{2} & 1.25 \hat{k}_{A} \Delta_{A}\left(K_{P}(A) A \cdot 1.25 \hat{k}_{P} \Delta_{A}+\Delta_{P}\right) \\
1.25 \hat{k}_{A} \Delta_{A}\left(K_{P}(A) A \cdot 1.25 \hat{k}_{P} \Delta_{A}+\Delta_{P}\right) & -\frac{1}{2}\left(K_{P}(A) A \cdot 1.25 \hat{k}_{P} \Delta_{A}+\Delta_{P}\right)^{2}
\end{array}\right] R^{2} \\
+\left[\begin{array}{cc}
-0.625 \hat{k}_{A} \Delta_{A}\left(K_{P}(A) A \cdot 1.25 \hat{k}_{P} \Delta_{A}+\Delta_{P}\right)^{2} & 0 \\
0 & -0.625 \hat{k}_{A} \Delta_{A}\left(K_{P}(A) A \cdot 1.25 \hat{k}_{P} \Delta_{A}+\Delta_{P}\right)^{2}
\end{array}\right] R^{3} .
\end{aligned}
$$

Hence, it is possible to describe the perturbed klystron model in the form of a multiplicative uncertain system

where

$$
\begin{aligned}
& \dot{x}=A_{k} x+B_{k} u \\
& y=\left(I+\Delta\left(A, \Delta_{A}, \Delta_{P}\right)\right) C_{k}(A) x
\end{aligned}
$$

$$
\begin{aligned}
\Delta\left(A, \Delta_{A}, \Delta_{P}\right)= & {\left[\begin{array}{cc}
1.25 \hat{k}_{A} \Delta_{A} & -\left(K_{P}(A) A \cdot 1.25 \hat{k}_{P} \Delta_{A}+\Delta_{P}\right) \\
\left(K_{P}(A) A \cdot 1.25 \hat{k}_{P} \Delta_{A}+\Delta_{P}\right) & 1.25 \hat{k}_{A} \Delta_{A}
\end{array}\right] R } \\
& +\left[\begin{array}{cc}
-\frac{1}{2}\left(K_{P}(A) A \cdot 1.25 \hat{k}_{P} \Delta_{A}+\Delta_{P}\right)^{2} & 1.25 \hat{k}_{A} \Delta_{A}\left(K_{P}(A) A \cdot 1.25 \hat{k}_{P} \Delta_{A}+\Delta_{P}\right) \\
1.25 \hat{k}_{A} \Delta_{A}\left(K_{P}(A) A \cdot 1.25 \hat{k}_{P} \Delta_{A}+\Delta_{P}\right) & -\frac{1}{2}\left(K_{P}(A) A \cdot 1.25 \hat{k}_{P} \Delta_{A}+\Delta_{P}\right)^{2}
\end{array}\right] R^{2} \\
& +\left[\begin{array}{cc}
-0.625 \hat{k}_{A} \Delta_{A}\left(K_{P}(A) A \cdot 1.25 \hat{k}_{P} \Delta_{A}+\Delta_{P}\right)^{2} & 0 \\
0 & -0.625 \hat{k}_{A} \Delta_{A}\left(K_{P}(A) A \cdot 1.25 \hat{k}_{P} \Delta_{A}+\Delta_{P}\right)^{2}
\end{array}\right] R^{3} .
\end{aligned}
$$


Equations (2.65) and (2.66) represent the linear parameter varying klystron model which needs estimation or measurement of the normalized input voltage $A$. Instead of $A$, the operating input voltage $A_{d}$ which is given by (2.38) is considered. Then, (2.65) and (2.66) are written by

$$
\begin{aligned}
& \dot{x}=A_{k} x+B_{k} u \\
& y=\left(I+\Delta\left(A_{d}, \Delta_{A}, \Delta_{P}\right)\right) C_{k}\left(A_{d}\right) x .
\end{aligned}
$$

The perturbed klystron model as given in (2.67) and (2.68) describes the klystron behavior around the wider neighborhood of the operating point $A_{d}$ than that of the Lyapunov linearization.

The transfer matrix of the perturbed klystron due to the high voltage power supply ripple is expressed in the form of a multiplicative uncertainty.

$$
G_{P}(S)=\left(I+\Delta\left(A_{d}, \Delta_{A}, \Delta_{P}\right)\right) G(s)
$$

where

$$
G(s)=C_{k}\left(A_{d}\right)\left(s I-A_{k}\right)^{-1} B_{k} .
$$

It is also possible to express the transfer matrix of the perturbed klystron in the form of an additive uncertainty.

$$
\begin{aligned}
G_{P}(S) & =\left(I+\Delta\left(A_{d}, \Delta_{A}, \Delta_{P}\right)\right) G(s) \\
& =G(s)+\Delta\left(A_{d}, \Delta_{A}, \Delta_{P}\right) G(s) .
\end{aligned}
$$

The additive uncertainty system (2.71) can give the information of the effect of high voltage power supply ripple on the output of klystron more directly. Figure 2.21 shows the multiplicative uncertainty representation of the high voltage power supply ripple and figure 2.22 shows the additive uncertainty representation of the high voltage power supply ripple.

In order to represent the perturbed system (2.69) or (2.71) in the standard form of an uncertainty, $\Delta\left(A, \Delta_{A}, \Delta_{P}\right)$ is to be described as

$$
\Delta\left(A, \Delta_{A}, \Delta_{P}\right)=W_{\text {Ripple }}(s) \bar{\Delta}_{R}\left(A, \Delta_{A}, \Delta_{P}\right)
$$

where

$$
\left\|\bar{\Delta}_{R}\left(A, \Delta_{A}, \Delta_{P}\right)\right\|_{\infty} \leq 1, \quad \forall \omega
$$

and the weighting function matrix $W_{\text {Ripple }}(s)$ is determined so that it reflects all frequency components that are in $\Delta\left(A, \Delta_{A}, \Delta_{P}\right)$.

A way to obtain (2.72) is as follows.

1. For $-1.0 \leq R \leq 1.0$, obtain the frequency response $G_{P}(j \omega), \forall \omega$.

2. Obtain

$$
l_{O}(j \omega)=\max _{\omega}\left|G^{-1}(j \omega)\left(G_{P}(j \omega)-G(j \omega)\right)\right|
$$


3. $W_{\text {Ripple }}(s)$ is determined such that

$$
\begin{aligned}
& W_{\text {Ripple }}(s)=\left[\begin{array}{cc}
w_{\text {Ripple }}(s) & 0 \\
0 & w_{\text {Ripple }}(s)
\end{array}\right] \\
& w_{\text {Ripple }}(j \omega) \geq l_{O}(j \omega), \quad \forall \omega .
\end{aligned}
$$

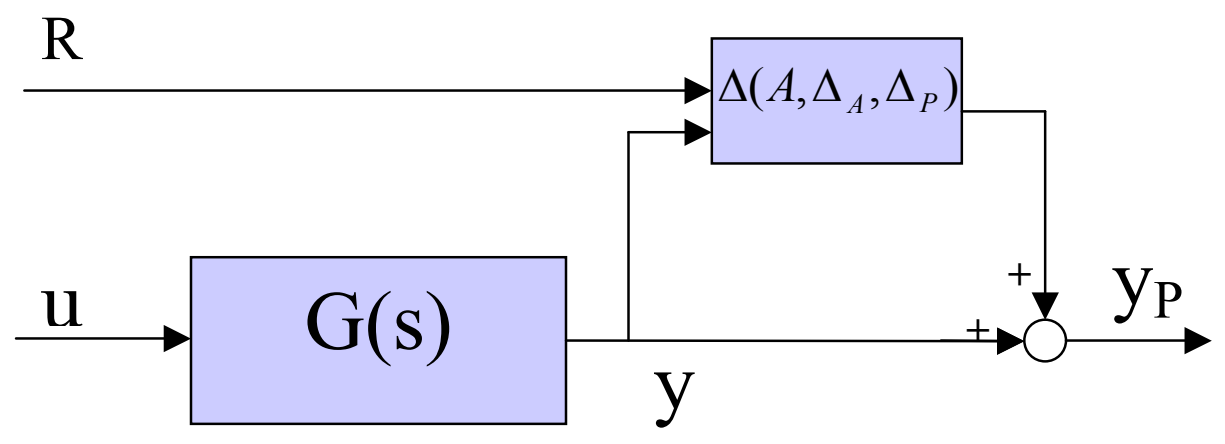

Figure 2.21 Multiplicative Uncertainty

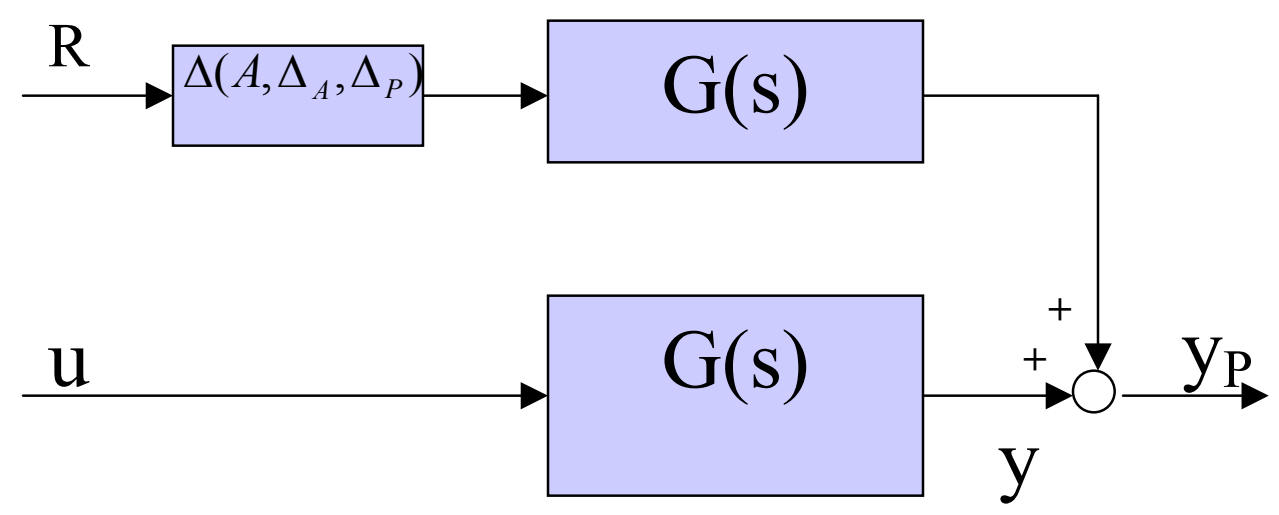

Figure 2.22 Additive Uncertainty 
Figure 2.23 shows $G_{P}(j \omega)$ for the $3^{\text {rd }}$ klystron for the $3^{\text {rd }}$ DTL tank when the ripple $R$ has the value in the range of $-1.0 \leq R \leq 1.0$. The nominal klystron $G(j \omega)$ is considered when $R=0.0$. Figure 2.24 shows $\left|G^{-1}(j \omega)\left(G_{P}(j \omega)-G(j \omega)\right)\right|,-1.0 \leq R \leq 1.0$. Based on the results in figure 2.24 , the weighting function matrix $W_{\text {Ripple }}(s)$ determined satisfying (2.74). Figure 2.25 shows the multiplicative uncertainty reprsentation of the perturbed klystron model due to high voltage power supply ripple

$$
G_{P}(S)=\left(I+W_{\text {Ripple }}(s) G(s) \bar{\Delta}_{R}\left(A, \Delta_{A}, \Delta_{P}\right)\right) G(s)
$$

and figure 2.26 shows the additive uncertainty reprsentation of the perturbed klystron model due to high voltage power supply ripple

$$
\begin{aligned}
G_{P}(S) & =G(s)+W_{\text {Ripple }}(s) \bar{\Delta}_{R}\left(A, \Delta_{A}, \Delta_{P}\right) G(s) \\
& =G(s)+W_{\text {Ripple }}(s) G(s) \bar{\Delta}_{R}\left(A, \Delta_{A}, \Delta_{P}\right)
\end{aligned}
$$

When the weighting function matrix $W_{\text {Ripple }}(s)$ is diagonal,

$$
G_{P}(S)=G(s)+G(s) W_{\text {Ripple }}(s) \bar{\Delta}_{R}\left(A, \Delta_{A}, \Delta_{P}\right)
$$

Usually, the diagonal weighting function matrix is selected when the coupling terms among channels in the system are small. Figure 2.26 shows the additive uncertainty configuration of the perturbed klystron model. The signal level of $\bar{d}_{R}$ is $\left\|\bar{d}_{R}\right\|_{2} \leq 1$ and $\bar{d}_{R}$ contains the frequency components of the ripple. Since the perturbed klystron model in the additive uncertainty configuration gives the effect of the high voltage power supply ripple on the output of the klystron, it is to be used in the analysis of the low level RF system and the synthesis of a controller such as a PI feedback controller and feedforward controller of iterative learning control. 


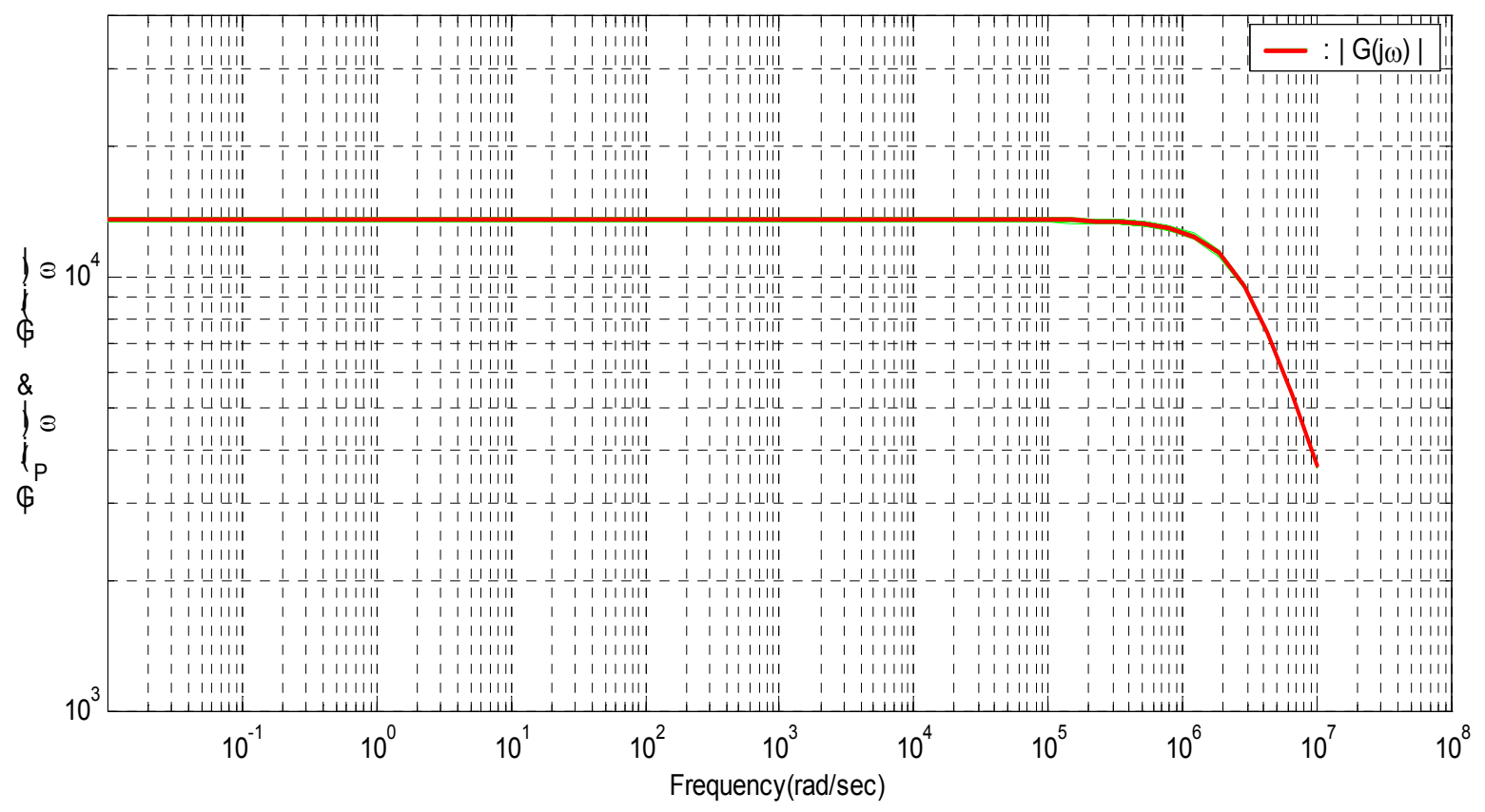

Figure 2.23 $G_{P}(j \omega),-1.0 \leq R \leq 1.0$

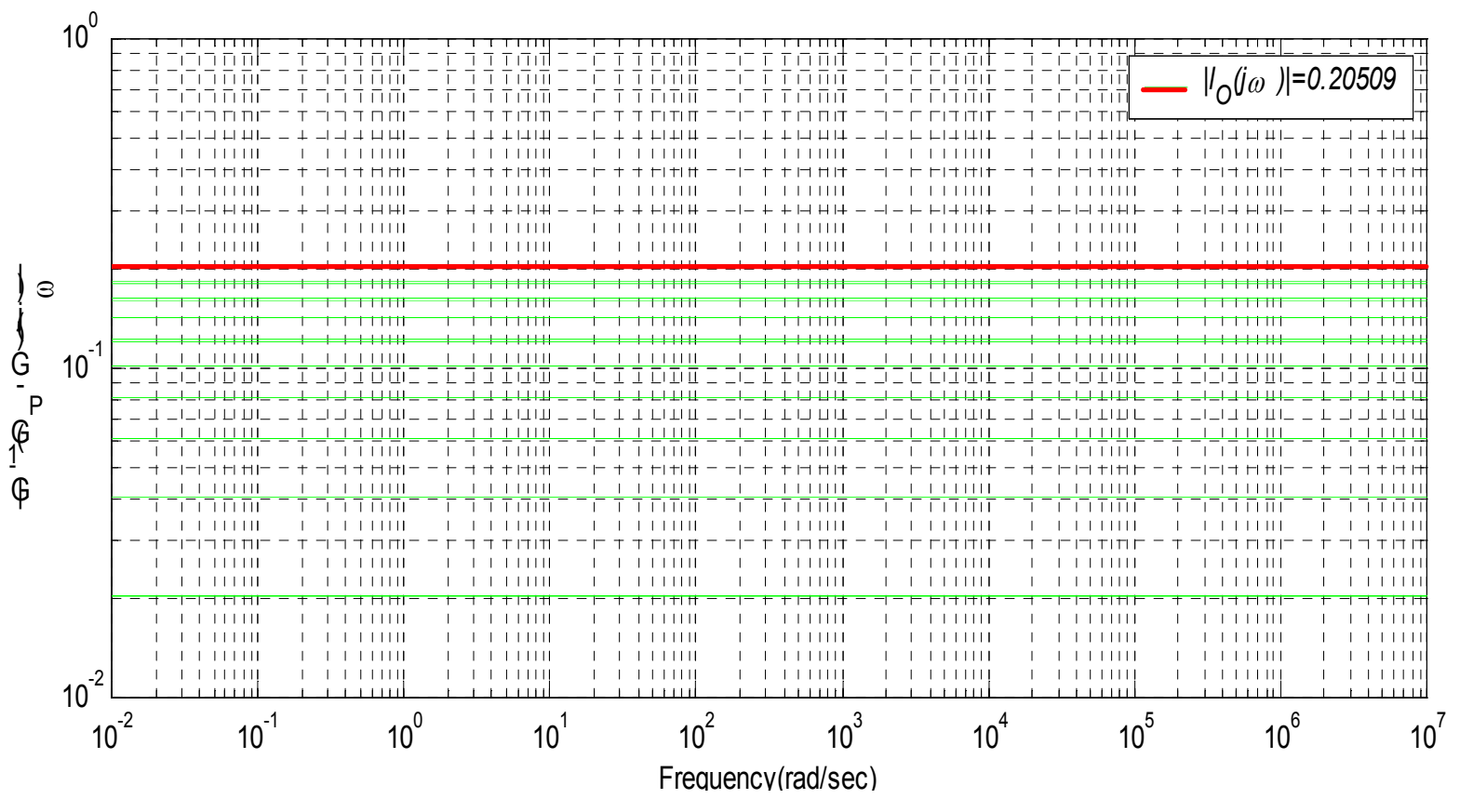

Figure 2.24 $\left|G^{-1}(j \omega)\left(G_{P}(j \omega)-G(j \omega)\right)\right|, \quad-1.0 \leq R \leq 1.0$ 


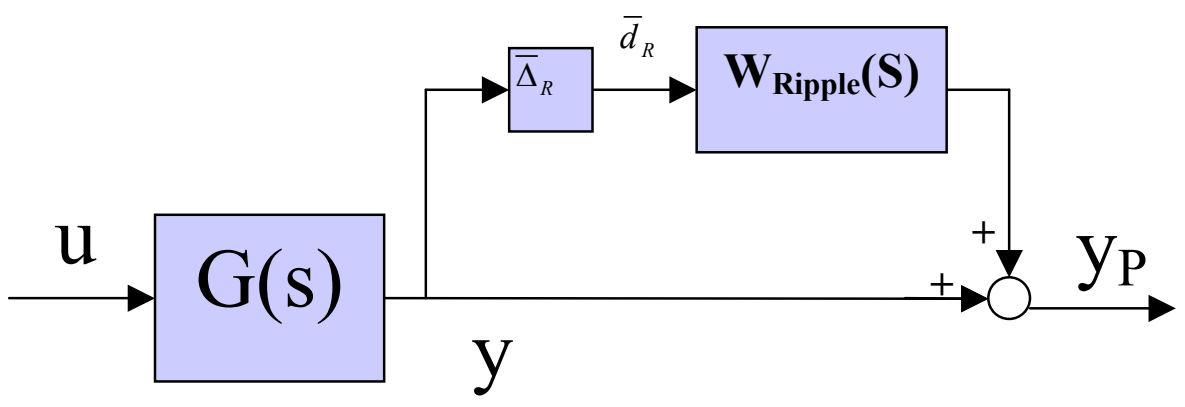

Figure 2.25 Standard Setup for Multiplicative Uncertainty
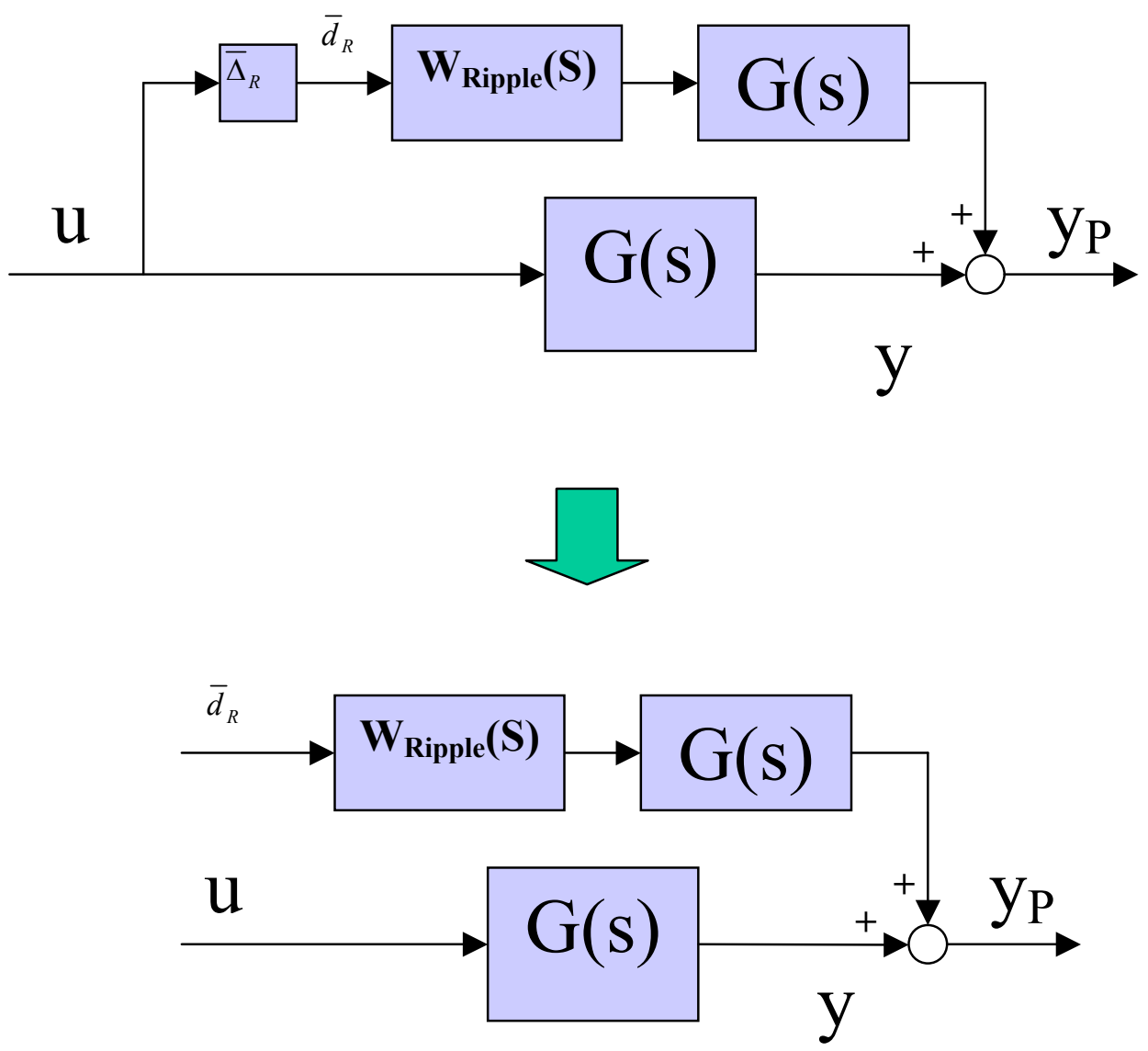

Figure 2.26 Standard Setup for Additive Uncertainty 


\section{CAVITY MODELS}

\section{III-A. SRF CAVITY MODEL}

The modeling of SRF cavity is based on the assumption that the RF generator and the cavity are connected by a transformer. The equivalent circuit of the cavity is transformed to the equivalent circuit of RF generator with a transmission line (wave guide) and the model is obtained $[4,15]$.

The SRF cavity can be represented by the state space equation (controllable canonical form [6]).

$$
\begin{aligned}
{\left[\begin{array}{l}
\dot{x}_{1} \\
\dot{x}_{2} \\
\dot{x}_{3} \\
\dot{x}_{4}
\end{array}\right]=} & {\left[\begin{array}{cccc}
0 & 1 & 0 & 0 \\
-\left(b_{2}+\frac{1}{R_{o}} c_{1} a_{1 c}\right) & -\left(b_{1}+\frac{1}{R_{o}} c_{1}\right) & \frac{1}{R_{o}} c_{3} a_{1 s} & \frac{1}{R_{o}} c_{3} \\
0 & 0 & 0 & 1 \\
-\frac{1}{R_{o}} c_{3} a_{1 s} & -\frac{1}{R_{o}} c_{3} & -\left(b_{2}+\frac{1}{R_{o}} c_{1} a_{1 c}\right) & -\left(b_{1}+\frac{1}{R_{o}} c_{1}\right)
\end{array}\right]\left[\begin{array}{l}
x_{1} \\
x_{2} \\
x_{3} \\
x_{4}
\end{array}\right] } \\
& +\left[\begin{array}{cc}
0 & 0 \\
\frac{2}{R_{o}} & 0 \\
0 & 0 \\
0 & \frac{2}{R_{o}}
\end{array}\right]\left[\begin{array}{l}
V_{f I} \\
V_{f Q}
\end{array}\right]+\left[\begin{array}{cc}
0 & 0 \\
-2 \zeta & 0 \\
0 & 0 \\
0 & -2 \zeta
\end{array}\right]\left[\begin{array}{l}
I_{I} \\
I_{Q}
\end{array}\right] \\
{\left[\begin{array}{l}
V_{I} \\
V_{Q}
\end{array}\right]=} & {\left[\begin{array}{cccc}
c_{1} a_{1 c} & c_{1} & -c_{3} a_{1 s} & -c_{3} \\
c_{3} a_{1 s} & c_{3} & c_{1} a_{1 c} & c_{1}
\end{array}\right]\left[\begin{array}{l}
x_{1} \\
x_{2} \\
x_{3} \\
x_{4}
\end{array}\right] }
\end{aligned}
$$

where

$$
\begin{aligned}
& a_{1 c}=\frac{1}{\tau}-\frac{\left(\Delta \omega_{m}+\Delta \omega_{L}\right)}{2 Q_{o}}, \quad a_{1 s}=\frac{1}{\tau}+2 Q_{o}\left(\Delta \omega_{m}+\Delta \omega_{L}\right) \\
& b_{1}=\frac{2}{\tau}, \quad b_{2}=\frac{1}{\tau^{2}}+\left(\Delta \omega_{m}+\Delta \omega_{L}\right)^{2}, \quad c_{1}=\frac{R_{c u}}{\tau}, \quad c_{3}=\frac{R_{c u}}{2 Q_{o} \tau}
\end{aligned}
$$

$\tau=\frac{2 Q_{o}}{\omega_{o}}:$ Unloaded cavity damping time constant $[\mathrm{s}]$

$Q_{o}$ : Cavity resonator unloaded quality factor

$\omega_{o}:$ Cavity resonance frequency $[\mathrm{rad} / \mathrm{s}]$

$R_{c u}:$ Resistance of the equivalent circuit of cavity transformed

$$
\text { to RF generator }
$$

$R_{o}$ : Transmission Line characteristic impedance

$\Delta \omega_{m}$ : Detuning frequency[rad/s] 


$$
\begin{aligned}
& \Delta \omega_{L}: \text { Lorentz Force Detuning } \\
& V_{f I}, V_{f Q}: \text { forward I/Q voltage } \\
& I_{I}, I_{Q}: \text { Beam current in I/Q } \\
& V_{I}, V_{Q}: \text { Cavity Field in I/Q } \\
& \zeta: \text { Transformation ratio. }
\end{aligned}
$$

In the state equation (3.1), the subsystems representing the input-states, $V_{f I}-\left[\begin{array}{l}x_{1} \\ x_{2}\end{array}\right], V_{f Q}-\left[\begin{array}{l}x_{3} \\ x_{4}\end{array}\right]$, are the controllable canonical forms, which reveal the dynamics of the In-Phase (I) and Quadrature (Q) channels, respectively, and the subsystems representing the input-states, $V_{f Q}-\left[\begin{array}{l}x_{1} \\ x_{2}\end{array}\right], V_{f I}-\left[\begin{array}{l}x_{3} \\ x_{4}\end{array}\right]$ represent the cross-coupling between the In-Phase (I) and Quadrature (Q) channels. The Lorentz Force Detuning dynamics is represented by a state equation given below.

$$
\begin{aligned}
& \dot{\Delta} \omega_{L}=-\frac{1}{\tau_{m}} \Delta \omega_{L}-\frac{2 \pi}{\tau_{m}} K E_{a c c}^{2} . \\
& E_{\text {acc }}: \text { the actual electric field intensity of the cavity }[\mathrm{MV} / \mathrm{m}] \\
& \tau_{m}: \text { the mechanical time constant of the cavity }[\mathrm{s}] \\
& K \text { : the Lorentz Force Detuning constant }\left[\left(\mathrm{Hz} /(\mathrm{MV} / \mathrm{m})^{2}\right)\right] .
\end{aligned}
$$

In order to incorporate the cavity field dynamics given by (3.1) and (3.2) to the Lorentz Froce Detuning dynamics, it is necessary to replace $E_{a c c}$ in (3.3) with

$$
\frac{E_{a c c}[M V / m]}{V_{g a p}[V]} \zeta \sqrt{V_{I}^{2}+V_{Q}^{2}}[V]
$$

where $\quad V_{g a p}[V]$ is the cavity gap voltage. Hence, we can write the Lorentz Force Detuning dynamics as

$$
\dot{\Delta} \omega_{L}=-\frac{1}{\tau_{m}} \Delta \omega_{L}-\frac{2 \pi}{\tau_{m}} K\left[\zeta \frac{E_{a c c}[M V / m]}{V_{g a p}[V]}\right]^{2}\left(V_{I}^{2}+V_{Q}^{2}\right) .
$$

Define

$$
\bar{K}=K\left[\zeta \frac{E_{o}[M V / m]}{V_{g a p}[V]}\right]^{2}
$$

Then, the Lorentz Force Detuning dynamics is described by

$$
\dot{\Delta} \omega_{L}=-\frac{1}{\tau_{m}} \Delta \omega_{L}-\frac{2 \pi}{\tau_{m}} \bar{K} V_{I}^{2}-\frac{2 \pi}{\tau_{m}} \bar{K} V_{Q}^{2}
$$


The open loop system as given in (3.1) and (3.2) is time varying (parameter varying) since Lorentz Force Detuning $\Delta \omega_{L}$ is varying. The system is written in the formal way of a linear time varying (parameter varying) state space equation.

$$
\begin{aligned}
& \dot{x}=A\left(\Delta \omega_{L}\right) x+B\left(\Delta \omega_{L}\right) u+B_{I}\left(\Delta \omega_{L}\right) I \\
& y=C\left(\Delta \omega_{L}\right) x \\
& A\left(\Delta \omega_{L}\right)=\left[\begin{array}{cccc}
0 & 1 & 0 & 0 \\
-\left(b_{2}+\frac{1}{R_{o}} c_{1} a_{1 c}\right) & -\left(b_{1}+\frac{1}{R_{o}} c_{1}\right) & \frac{1}{R_{o}} c_{3} a_{1 s} & \frac{1}{R_{o}} c_{3} \\
0 & 0 & 0 & 1 \\
-\frac{1}{R_{o}} c_{3} a_{1 s} & -\frac{1}{R_{o}} c_{3} & -\left(b_{2}+\frac{1}{R_{o}} c_{1} a_{1 c}\right) & -\left(b_{1}+\frac{1}{R_{o}} c_{1}\right)
\end{array}\right] \\
& B\left(\Delta \omega_{L}\right)=\left[\begin{array}{cc}
0 & 0 \\
\frac{2}{R_{o}} & 0 \\
0 & 0 \\
0 & \frac{2}{R_{o}}
\end{array}\right], B_{I}\left(\Delta \omega_{L}\right)=\left[\begin{array}{cc}
0 & 0 \\
-2 \zeta & 0 \\
0 & 0 \\
0 & -2 \zeta
\end{array}\right] \\
& C\left(\Delta \omega_{L}\right)=\left[\begin{array}{cccc}
c_{1} a_{1 c} & c_{1} & -c_{3} a_{1 s} & -c_{3} \\
c_{3} a_{1 s} & c_{3} & c_{1} a_{1 c} & c_{1}
\end{array}\right] \\
& x=\left[\begin{array}{llll}
x_{1} & x_{2} & x_{3} & x_{4}
\end{array}\right]^{T}, \quad u=\left[\begin{array}{ll}
V_{f I} & V_{f Q}
\end{array}\right]^{T}, \quad I=\left[\begin{array}{ll}
I_{I} & I_{Q}
\end{array}\right]^{T}, \quad y=\left[\begin{array}{ll}
V_{I} & V_{Q}
\end{array}\right]^{T} .
\end{aligned}
$$

Note that $C\left(\Delta \omega_{L}\right)$ and $B\left(\Delta \omega_{L}\right)$ are constant matrices and only $A\left(\Delta \omega_{L}\right)$ is the time varying (parameter varying).

It can be easily verified that the system as given in (3.5) and (3.6) is controllable but not observable for any $\Delta \omega_{L}$. The observability matrix of the system is given by

$$
O=\left[\begin{array}{c}
C\left(\Delta \omega_{L}\right) \\
C\left(\Delta \omega_{L}\right) A\left(\Delta \omega_{L}\right) \\
: \\
C\left(\Delta \omega_{L}\right) A^{n-1}\left(\Delta \omega_{L}\right)
\end{array}\right]
$$

whose rank is 2 for any $\Delta \omega_{L}$. Also, the rows of the output matrix $C\left(\Delta \omega_{L}\right)$ are linearly independent for any $\Delta \omega_{L}$. Hence, a nonsingular transformation matrix, $P$, can be defined where the first two rows of $P$ are the two rows of the output matrix $C\left(\Delta \omega_{L}\right)$ and the other two rows, $v_{1}, v_{2}$, are entirely arbitrary so long as $P$ is nonsingular.

$$
\left[\begin{array}{c}
z \\
z_{\bar{o}}
\end{array}\right]=\left[\begin{array}{c}
z_{1} \\
z_{2} \\
z_{\bar{o} 1} \\
z_{\bar{o} 2}
\end{array}\right]=P x=\left[\begin{array}{c}
C\left(\Delta \omega_{L}\right) \\
v_{1} \\
v_{2}
\end{array}\right]\left[\begin{array}{l}
x_{1} \\
x_{2} \\
x_{3} \\
x_{4}
\end{array}\right]
$$


Using the matrix $P$, the system given by (3.5) and (3.6) is transformed to the equivalent system in $\mathrm{z}$ coordinates.

$$
\begin{aligned}
& {\left[\begin{array}{c}
\dot{z} \\
\dot{z}_{\bar{o}}
\end{array}\right]=\left[\begin{array}{cc}
A_{z}\left(\Delta \omega_{L}\right) & 0 \\
\bar{A}_{21} & \bar{A}_{\bar{o}}
\end{array}\right]\left[\begin{array}{c}
z \\
z_{\bar{o}}
\end{array}\right]+\left[\begin{array}{c}
B_{z} \\
B_{\bar{o}}
\end{array}\right] u+\left[\begin{array}{c}
B_{z I} \\
B_{\bar{o} I}
\end{array}\right] I} \\
& y=\left[\begin{array}{ll}
C_{z} & 0
\end{array}\right]\left[\begin{array}{c}
z \\
z_{\bar{o}}
\end{array}\right]
\end{aligned}
$$

where

$$
\begin{aligned}
& A_{z}\left(\Delta \omega_{L}\right)=C\left(\Delta \omega_{L}\right) A\left(\Delta \omega_{L}\right) C^{+}\left(\Delta \omega_{L}\right)=\left[\begin{array}{cc}
-\frac{1}{\tau_{L}} & -\left(\Delta \omega_{m}+\Delta \omega_{L}\right) \\
\left(\Delta \omega_{m}+\Delta \omega_{L}\right) & -\frac{1}{\tau_{L}}
\end{array}\right] \\
& B_{z}=C\left(\Delta \omega_{L}\right) B\left(\Delta \omega_{L}\right)=\left[\begin{array}{cc}
\frac{2}{R_{o}} c_{1} & -\frac{2}{R_{o}} c_{3} \\
\frac{2}{R_{o}} c_{3} & \frac{2}{R_{o}} c_{1}
\end{array}\right] \\
& B_{z I}=C\left(\Delta \omega_{L}\right) B_{I}\left(\Delta \omega_{L}\right)=\left[\begin{array}{ll}
-2 c_{1} \zeta & 2 c_{3} \zeta \\
-2 c_{3} \zeta & -2 c_{1} \zeta
\end{array}\right] \\
& C_{z}=\left[\begin{array}{ll}
1 & 0 \\
0 & 1
\end{array}\right],
\end{aligned}
$$

and

$$
\begin{aligned}
& \tau_{L}=\frac{2 Q_{L}}{\omega_{o}}: \text { Loaded cavity damping constant, } \\
& Q_{L}=\frac{Q_{o}}{1+\beta}: \text { Loaded } Q \\
& \beta=1+\frac{P_{b}}{P_{c u}}: \text { Cavity coupling factor, }
\end{aligned}
$$

and $C^{+}\left(\Delta \omega_{L}\right)$ is the Moore-Penrose Pseudo-inverse[5] of $C\left(\Delta \omega_{L}\right)$.

In the system (3.8) and (3.9), the state $z_{\bar{o}}$ does not appear directly in the output $y$ or indirectly through $z$. Hence, the state $z_{\bar{o}}$ is not observable and is dropped in the reduced system given by

$$
\begin{aligned}
& \dot{z}=A_{z}\left(\Delta \omega_{L}\right) z+B_{z} u+B_{z I} I \\
& y=C_{z} z
\end{aligned}
$$

It can be easily verified that the reduced system (3.10) and (3.11) is also controllable and its input-output relation is given by

$$
Y(s)=C_{z}\left(s I-A_{z}\left(\Delta \omega_{L}\right)\right)^{-1} B_{z} U(s)+C_{z}\left(s I-A_{z}\left(\Delta \omega_{L}\right)\right)^{-1} I(s)
$$

where $U(s), Y(s)$, and $I(s)$ are Laplace transforms of $u(t), y(t)$, and $I(t)$, respectively. It can be easily verified that the input-output relation of the system given by (3.5) and (3.6) is the same as the 
equation (3.12). Since the reduced system (3.10) and (3.11) is both controllable and observable, it is irreducible, which means physically that the dimension of the reduced system is the minimal number of integrators or the minimal number of energy-storage elements required to generate the given transfer matrix. Figure 3.1 shows the frequency response of the system (3.10) and (3.11). Meanwhile, the Lorentz Force Detuning (3.4) is written as

$$
\dot{\Delta} \omega_{L}=-\frac{1}{\tau_{m}} \Delta \omega_{L}-\frac{2 \pi}{\tau_{m}} \bar{K} z_{1}^{2}-\frac{2 \pi}{\tau_{m}} \bar{K} z_{2}^{2}
$$

The system representation in frequency domain as given in (3.12) can be expressed

$$
Y(s)=G_{c}(s) U(s)+G_{B}(s) I(s)
$$

where

$$
\begin{aligned}
& G_{c}(s)=C_{z}\left(s I-A_{z}\left(\Delta \omega_{L}\right)\right)^{-1} B_{z}, \\
& G_{B}(s)=C_{z}\left(s I-A_{z}\left(\Delta \omega_{L}\right)\right)^{-1} B_{z I} .
\end{aligned}
$$

The equation (3.14) shows that from the perspective of a cavity, beam current is an exogenous disturbance. Also, the coefficients of the transfer matrix are dependent upon the Lorentz Force Detuning $\Delta \omega_{L}$.

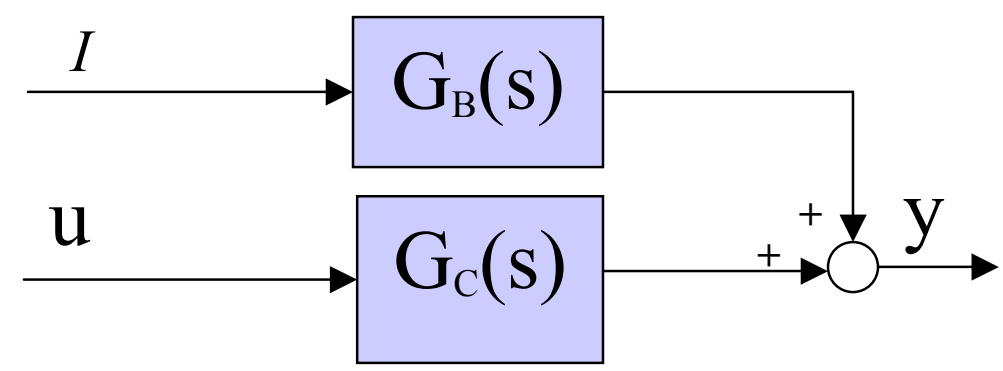

Figure 3.1 Cavity Model. Beam current $I$ is an exogenous disturbance

Table 3.1 shows the SRF parameters for cavity \#70. Based on these parameters, the SRF cavity is modeled in the form of state space model or transfer matrix. In SNS, there are 81 SRF cavities. In order to model 81 SRF cavities, parameters as shown in table 3.1 are calculated for each SRF cavity. Then, the mathematical model is obtained. Figure 3.2 shows the frequency responses of $G_{C}(s)$ 's of $81 \mathrm{SRF}$ cavities and figure 3.3 shows the frequency responses of $G_{B}(s)$ 's when the Lorentz Force Detuning $\Delta \omega_{L}$ is assumed zero. 
Table 3.1 SRF cavity $\# 70$ parameters, power control margin $=0.33(33 \%$, assumed $)$

\begin{tabular}{|c|c|}
\hline$P_{b}, \quad$ Beam Power & $367 \mathrm{~kW}$ \\
\hline$\phi_{S}, \quad$ Synchronous phase & $-19.5^{\circ}$ \\
\hline$E_{a c c}, \quad$ Electric Field & $15.8443 \mathrm{MV} / \mathrm{m}$ \\
\hline$K, \quad$ Lorentz Force Detuning constant & $-2.0\left(\mathrm{~Hz} /(\mathrm{MV} / \mathrm{m})^{2}\right.$ \\
\hline $\bar{K}=K\left(\varsigma \frac{E_{a c c}[M V / m]}{V_{g a p}[V]}\right)^{2}$ & $-1.3679 \mathrm{e}-005$ \\
\hline$\tau_{m}, \quad$ Mechanical time constant & $1.0 \mathrm{msec}$ \\
\hline$Q_{o}, \quad$ Unloaded quality factor & $3.0 e+009$ \\
\hline$I_{b}, \quad$ Avrage beam current & $26 m A$ \\
\hline$f_{o}, \quad$ Resonance frequency & $805 \mathrm{MHz}$ \\
\hline$\omega_{o}, \quad$ Cavity resonance frequency & $5.0580 \mathrm{e}+009 \mathrm{rad} / \mathrm{sec}$ \\
\hline$P_{c u}, \quad$ Cavity wall dissipation & $51.2780 \mathrm{~W}$ \\
\hline$P_{g}^{P e a k}=\left(P_{b}+P_{c u}\right) \cdot(1+$ Power Control Margin), Generator peak power & $488.18 \mathrm{~kW}$ \\
\hline$R_{O}, \quad$ Transmission line impedance & $50 \Omega$ \\
\hline$V_{\text {gap }}=\frac{P_{b}}{I_{b} \cos \left(\phi_{S}\right)}, \quad$ Peak cavity voltage & $14.974 M V$ \\
\hline$L_{\text {cav }}, \quad$ Cavity Length & $0.9451 \mathrm{~m}$ \\
\hline$V_{b}=V_{\text {gap }} \cos \left(\phi_{s}\right), \quad$ Beam Voltage & $14.1154 \mathrm{MV}$ \\
\hline$P_{f}=P_{b}+P_{c u}, \quad$ Forward Power & $367.0513 \mathrm{~kW}$ \\
\hline$V_{f}=\sqrt{2 R_{o} \cdot P_{f}}, \quad$ Forward Voltage & $6058.5 \mathrm{~V}$ \\
\hline$I_{f}=\frac{V_{f}}{R_{O}}$, Forward Current & $121.1695 \mathrm{~A}$ \\
\hline$\Delta \omega_{m}=\frac{V_{g a p}}{2 P_{c u}} \cdot \mathrm{I}_{\mathrm{b}} \cdot \frac{\omega_{\mathrm{o}}}{\mathrm{Q}_{\mathrm{o}}} \cdot \sin \left(\frac{\pi}{180} \phi_{S}\right), \quad$ Detuning frequency & $-1281.9 \mathrm{rad} / \mathrm{sec}$ \\
\hline$\psi_{m}=-\tan ^{-1}\left(\frac{2 Q_{L} \cdot \Delta \omega_{m}}{\omega_{o}}\right), \quad$ Detuning angle & $19.4840^{\circ}$ \\
\hline$\beta=1+\frac{P_{b}}{P_{c u}}$, Cavity coupling factor & 7160 \\
\hline$Q_{L}=\frac{Q_{o}}{1+\beta}$, Loaded quality factor & $6.98 \mathrm{e}+005$ \\
\hline$\tau=\frac{2 Q_{O}}{\omega_{O}}, \quad$ Cavity (unloaded) damping time constant & $1.1862 \mathrm{sec}$ \\
\hline
\end{tabular}




\begin{tabular}{|c|c|}
\hline$\tau_{L}=\frac{2 Q_{L}}{\omega_{o}}$, Loaded time constant & $0.2760 \mathrm{msec}$ \\
\hline$R_{s h}=\frac{V_{g a p}^{2}}{P_{c u}}$, Shunt impedance & $4.3728 \mathrm{e}+012 \Omega$ \\
\hline$R_{c}=\frac{R_{s h}}{2}, \quad$ Cavity resistance & $2.1864 \mathrm{e}+012 \Omega$ \\
\hline$\zeta=\sqrt{\frac{R_{C}}{\beta R_{o}}}, \quad$ Transformer ratio & 2471.6 \\
\hline $\begin{array}{l}R_{c u}=\frac{\mathrm{R}_{\mathrm{c}}}{\zeta^{2}}, \quad \text { Cavity resistance transformed to the equivalent circuit of } \mathrm{RF} \\
\text { generator }\end{array}$ & $3.5790 \mathrm{e}+005 \mathrm{~s}$ \\
\hline$V_{b i}=I_{b} \cdot R_{s h} \cdot \cos (\psi) /(1+\beta), \quad$ Beam induced Voltage & $2.8223 \mathrm{e}+007 V$ \\
\hline$P_{g}=P_{f}, \quad$ Generator Power for Matched Cavity & $367.0513 \mathrm{~kW}$ \\
\hline$V_{g i}=2 \sqrt{P_{g} \cdot \beta \cdot R_{s h}} \cos (\psi) /(1+\beta)$, Generator induced Voltage & $1.9957 \mathrm{e}+007 V$ \\
\hline$I_{g e n}=2 \sqrt{P_{g} \cdot \beta / R_{s h}}$ & $0.0490 \mathrm{~A}$ \\
\hline
\end{tabular}

\begin{tabular}{|l|l|}
\hline$c_{1}=\frac{R_{c u}}{\tau}$ & $3.0172 \mathrm{e}+005$ \\
\hline$c_{3}=\frac{R_{c u}}{2 Q_{o} \tau}$ & $5.0287 \mathrm{e}-005$ \\
\hline
\end{tabular}



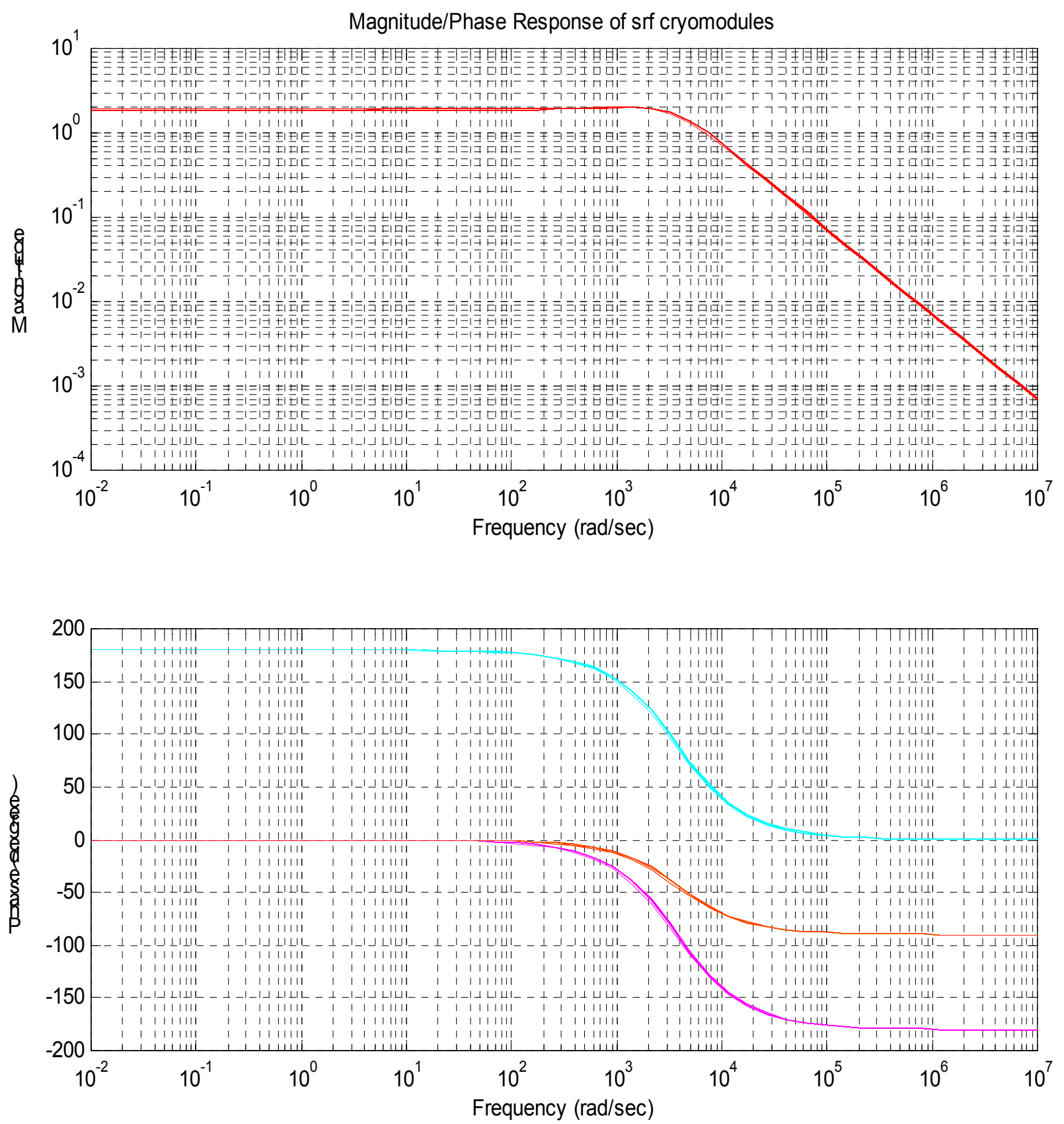

Figure 3.2 Frequency Responses of $81 \mathrm{SRF}$ cavities, $\left|G_{C}(j \omega)\right|$ and $\angle G_{C}(j \omega)$ 

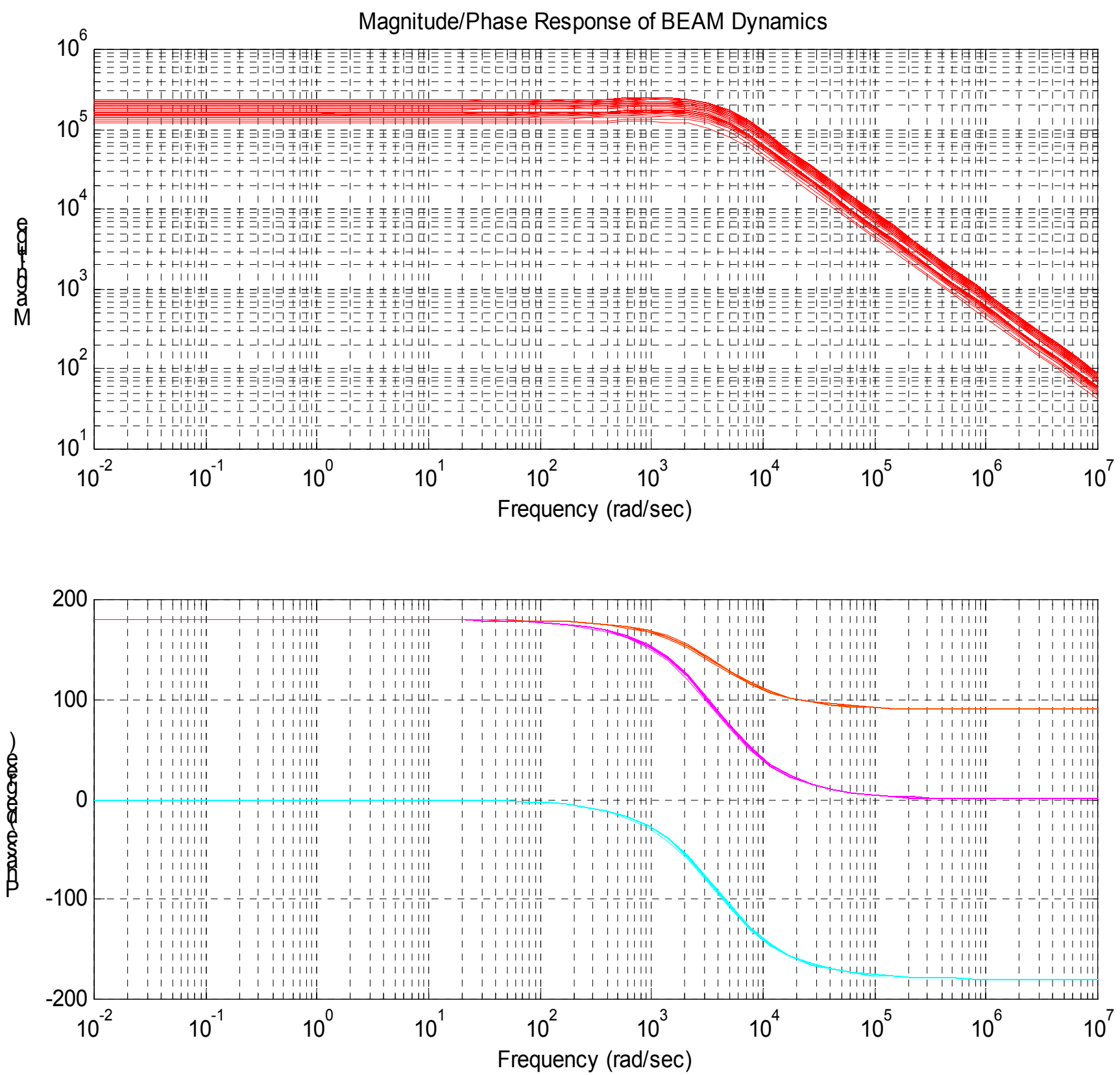

Figure 3.3 Frequency Responses of Beam Dynamics for 81 SRF cavities, $\left|G_{B}(j \omega)\right|$ and $\angle G_{B}(j \omega)$ 


\section{III-B. PERTURBED SRF CAVITY MODEL}

The dominant perturbation among the SRF cavity parameters is the external $\mathrm{Q}, Q_{\text {ext }}$. The loaded $\mathrm{Q}, Q_{L}$, is given by

$$
\frac{1}{Q_{L}}=\frac{1}{Q_{o}}+\frac{1}{Q_{e x t}}
$$

Since $Q_{o} \gg Q_{\text {ext }}$ in SRF cavity,

$$
Q_{L} \approx Q_{\text {ext }} .
$$

The relation between the load $\mathrm{Q}, Q_{L}$ and the coupling factor $\beta(\beta>>1)$ is given by

$$
Q_{L}=\frac{Q_{o}}{1+\beta} \approx \frac{Q_{o}}{\beta}
$$

Hence, the perturbation of the external Q, $Q_{e x t}$ is equivalently described by the inverse of the perturbation of the coupling factor $\beta$.

Let $\beta_{o}$ represent the nominal value of the coupling factor. Then a multiplicative perturbation of $\beta$ is expressed as

$$
\beta=\beta_{o}\left(1+\delta_{\beta}\right)
$$

where $\left|\delta_{\beta}\right| \leq 1.0$ represents the degree of the perturbation. For example, if $\beta$ is perturbed $+10.0 \%$ from its nominal value $\beta_{o}$, then $\delta_{\beta}=+0.1$ and $\beta=\beta_{o}(1+0.1)=1.1 \beta_{o}$.

We need to investigate the effect of perturbation of $\beta$ on the system matrices as given in (3.10)-(3.11) and the perturbed system. The system matrices are given by $\tau_{L}, c_{1}, c_{3}, \Delta \omega_{m}$, and $\zeta$. We ignore the effect of perturbation of $\beta$ on the Lorentz Force Detuning $\Delta w_{L}$.

First note that

$$
\frac{1}{\tau_{L}}=\frac{\omega_{o}}{2 Q_{L}}=\frac{\omega_{o}}{2 Q_{o} /(1+\beta)}=\frac{\omega_{o}}{2 Q_{o}}(1+\beta)
$$

Since for a SRF cavity $\beta>>1$, (3.16) can be written as

$$
\frac{1}{\tau_{L}} \approx \frac{\omega_{o}}{2 Q_{o}} \beta
$$

Also, since $\zeta=\sqrt{\frac{R_{c}}{\beta R_{O}}}, \tau=\frac{2 Q_{o}}{w_{o}}$, and $Q_{o}=(1+\beta) Q_{L}$,

$$
c_{1}=\frac{R_{c u}}{\tau}=\frac{R_{c} / \zeta^{2}}{2 Q_{o} / \omega_{o}}=\frac{\beta R_{O}}{2 Q_{o} / \omega_{o}},
$$




$$
c_{3}=\frac{R_{c u}}{2 Q_{o} \tau}=\frac{1}{2 Q_{o}} c_{1}=\frac{\beta R_{O}}{4 Q_{o}^{2} / w_{o}}
$$

Since $P_{b}=V_{g a p} \cdot I_{b} \cdot \cos \left(\phi_{s}\right), P_{b}=(\beta-1) P_{c u}$, and so $V_{g a p}=\frac{P_{b}}{I_{b} \cdot \cos \left(\phi_{s}\right)}=\frac{(\beta-1) P_{c u}}{I_{b} \cdot \cos \left(\phi_{s}\right)}$,

$$
\Delta \omega_{m}=\frac{V_{g a p}}{2 P_{c u}} \cdot \mathrm{I}_{\mathrm{b}} \cdot \frac{\omega_{\mathrm{o}}}{\mathrm{Q}_{\mathrm{o}}} \cdot \sin \left(\phi_{s}\right)=\frac{1}{2}(\beta-1) \cdot \frac{\omega_{\mathrm{o}}}{\mathrm{Q}_{\mathrm{o}}} \cdot \tan \left(\phi_{s}\right)
$$

Since $\beta>>1$, we can write (3.22) as

$$
\Delta \omega_{m} \approx \frac{1}{2} \beta \cdot \frac{\omega_{\mathrm{o}}}{\mathrm{Q}_{\mathrm{o}}} \cdot \tan \left(\phi_{s}\right)
$$

Since $\quad V_{g a p}=\frac{(\beta-1) P_{c u}}{I_{b} \cdot \cos \left(\phi_{s}\right)}$

$$
\begin{aligned}
& R_{s h}=\frac{V_{g a p}^{2}}{P_{c u}}=\frac{(\beta-1)^{2} P_{c u}}{I_{b}^{2} \cdot \cos ^{2}\left(\phi_{s}\right)}, \\
& R_{c}=\frac{R_{s h}}{2}=\frac{(\beta-1)^{2} P_{c u}}{2 I_{b}^{2} \cdot \cos ^{2}\left(\phi_{s}\right)} .
\end{aligned}
$$

Hence,

$$
\zeta=\sqrt{\frac{R_{c}}{\beta R_{O}}}=\sqrt{\frac{(\beta-1)^{2} P_{c u}}{2 \beta R_{O} I_{b}^{2} \cdot \cos ^{2}\left(\phi_{s}\right)}}=\sqrt{\frac{P_{c u}}{2 R_{O} I_{b}^{2} \cdot \cos ^{2}\left(\phi_{s}\right)}} \cdot \sqrt{\frac{(\beta-1)^{2}}{\beta}}
$$

and for $\beta>>1$,

$$
\zeta \approx \sqrt{\frac{P_{c u}}{2 R_{O} I_{b}^{2} \cdot \cos ^{2}\left(\phi_{s}\right)}} \cdot \sqrt{\beta}
$$

Let $\frac{1}{\tau_{L 0}}, c_{10}, c_{30}, \Delta \omega_{m 0}$, and $\zeta_{0}$ be the nominal values of $\frac{1}{\tau_{L}}, c_{1}, c_{3}, \Delta \omega_{m}$, and $\zeta$ corresponding to the nominal value $\beta_{o}$ of $\beta$ given by

$$
\begin{array}{lcl}
\frac{1}{\tau_{L 0}} \approx \frac{\omega_{o}}{2 Q_{o}} \beta_{o}, & c_{10}=\frac{\beta_{o} R_{O}}{2 Q_{o} / \omega_{o}}, & c_{30}=\frac{\beta_{o} R_{O}}{4 Q_{o}^{2} / \omega_{o}}, \\
\Delta \omega_{m 0} \approx \frac{1}{2} \beta_{o} \cdot \frac{\omega_{\mathrm{o}}}{\mathrm{Q}_{\mathrm{o}}} \cdot \tan \left(\phi_{s}\right), & \zeta_{0} \approx \sqrt{\frac{P_{c u}}{2 R_{O} I_{b}^{2} \cdot \cos ^{2}\left(\phi_{s}\right)}} \cdot \sqrt{\beta_{o}} .
\end{array}
$$


Then, for the perturbed value $\beta=\beta_{o}\left(1+\delta_{\beta}\right)$ of $\beta$ with $\left|\delta_{\beta}\right| \leq 1.0$,

$$
\begin{aligned}
& \frac{1}{\tau_{L}} \approx \frac{1}{\tau_{L 0}}\left(1+\delta_{\beta}\right), \\
& c_{1}=c_{10}\left(1+\delta_{\beta}\right), \\
& c_{3}=c_{30}\left(1+\delta_{\beta}\right) \\
& \Delta \omega_{m 0} \approx \Delta \omega_{m 0}\left(1+\delta_{\beta}\right), \\
& \zeta \approx \zeta_{0} \sqrt{1+\delta_{\beta}} .
\end{aligned}
$$

By using the perturbed parameters as given in (3.28)-(3.32), we can express the perturbed system matrices of the cavity model as

$$
\begin{aligned}
& A_{z}\left(\Delta w_{L}, \delta_{\beta}\right)=\left[\begin{array}{cc}
-\frac{1}{\tau_{L 0}} & -\Delta \omega_{m 0} \\
\Delta \omega_{m 0} & -\frac{1}{\tau_{L 0}}
\end{array}\right]\left(1+\delta_{\beta}\right)+\left[\begin{array}{cc}
0 & -\Delta \omega_{L} \\
\Delta \omega_{L} & 0
\end{array}\right], \\
& B_{z}\left(\delta_{\beta}\right)=\left[\begin{array}{cc}
\frac{2}{Z_{o}} c_{10} & -\frac{2}{Z_{o}} c_{30} \\
\frac{2}{Z_{o}} c_{30} & \frac{2}{Z_{o}} c_{10}
\end{array}\right]\left(1+\delta_{\beta}\right), \\
& B_{z I}\left(\delta_{\beta}\right)=\left[\begin{array}{ll}
-2 c_{10} \zeta_{0} & 2 c_{30} \zeta_{0} \\
-2 c_{30} \zeta_{0} & -2 c_{10} \zeta_{0}
\end{array}\right]\left(1+\delta_{\beta}\right)^{3 / 2} \approx\left[\begin{array}{cc}
-2 c_{10} \zeta_{0} & 2 c_{30} \zeta_{0} \\
-2 c_{30} \zeta_{0} & -2 c_{10} \zeta_{0}
\end{array}\right]\left(1+\frac{3}{2} \delta_{\beta}\right), \\
& C_{z}=\left[\begin{array}{ll}
1 & 0 \\
0 & 1
\end{array}\right] .
\end{aligned}
$$

In (3.33), the Lorentz Force Detuning $\Delta \omega_{L}$ is treated as the system matrix perturbation. The same effect is contributed by the microphonics, $\Delta \omega_{M C P}$. In this case, (3.33) is given by

$$
A_{z}\left(\Delta w_{L}, \Delta \omega_{m c p}, \delta_{\beta}\right)=\left[\begin{array}{cc}
-\frac{1}{\tau_{L 0}} & -\Delta \omega_{m 0} \\
\Delta \omega_{m 0} & -\frac{1}{\tau_{L 0}}
\end{array}\right]\left(1+\delta_{\beta}\right)+\left[\begin{array}{cc}
0 & -\left(\Delta \omega_{L}+\Delta \omega_{M C P}\right) \\
\left(\Delta \omega_{L}+\Delta \omega_{M C P}\right) & 0
\end{array}\right] .
$$

Consider a system

$$
\begin{aligned}
& \dot{z}=A_{z}\left(\Delta w_{L}, \Delta \omega_{M C P}, \delta_{\beta}\right) z+B_{z}\left(\delta_{\beta}\right) u \\
& y=C_{z} z
\end{aligned}
$$

This system is the nominal one with respect to the beam current $I$. 
Assume that the minimum value and the maximum value of the Lorentz Force Detuning $\Delta \omega_{L}$ are given by $\Delta \underline{\omega}_{L}$ and $\Delta \bar{\omega}_{L}$, respectively, and the minimum value and the maximum value of the microphonic $\Delta \omega_{M C P}$ are given by $\Delta \underline{\omega}_{M C P}$ and $\Delta \bar{\omega}_{M C P}$, respectively. The Lorentz Force Detuning $\Delta \omega_{L}$ and of the microphonic $\Delta \omega_{M C P}$ are scaled such that

$$
\begin{gathered}
\Delta \omega_{L}=\Delta \omega_{L n}+\Delta \omega_{L s} \Delta_{L F D} \\
\Delta \omega_{M C P}=\Delta \omega_{M C P n}+\Delta \omega_{M C P S} \Delta_{M C P} \\
\left\|\Delta_{L F D}\right\|_{2} \leq 1 \\
\left\|\Delta_{M C P}\right\| \leq 1
\end{gathered}
$$

where $\Delta \omega_{L n}$ and $\Delta \omega_{M C P n}$ are nominal values and $\Delta \omega_{L s}$ and $\Delta \omega_{M C P S}$ are scaling factors and

$$
\begin{aligned}
& \Delta \omega_{L n}=\frac{1}{2}\left(\Delta \underline{\omega}_{L}+\Delta \bar{\omega}_{L}\right) \\
& \Delta \omega_{M C P n}=\frac{1}{2}\left(\Delta \underline{\omega}_{M C P}+\Delta \bar{\omega}_{M C P}\right) \\
& \Delta \omega_{L s}=\frac{1}{2}\left(\Delta \bar{\omega}_{L}-\Delta \underline{\omega}_{L}\right) \\
& \Delta \omega_{M C P s}=\frac{1}{2}\left(\Delta \bar{\omega}_{M C P}-\Delta \underline{\omega}_{M C P}\right) .
\end{aligned}
$$

Then, $A_{z}\left(\Delta w_{L}, \Delta \omega_{m c p}, \delta_{\beta}\right)$ and $B_{z}\left(\delta_{\beta}\right)$ are decomposed as

$$
\begin{aligned}
& A_{z}\left(\Delta w_{L}, \Delta \omega_{m c p}, \delta_{\beta}\right)=\left[\begin{array}{cc}
-\frac{1}{\tau_{L 0}} & -\Delta \omega_{m 0} \\
\Delta \omega_{m 0} & -\frac{1}{\tau_{L 0}}
\end{array}\right]+\delta_{\beta}\left[\begin{array}{cc}
-\frac{1}{\tau_{L 0}} & -\Delta \omega_{m 0} \\
\Delta \omega_{m 0} & -\frac{1}{\tau_{L 0}}
\end{array}\right]+\Delta \omega_{L n}\left[\begin{array}{cc}
0 & -1 \\
1 & 0
\end{array}\right]+\Delta_{L F D}\left[\begin{array}{cc}
0 & -\Delta \omega_{L s} \\
\Delta \omega_{L s} & 0
\end{array}\right] \\
& +\Delta \omega_{M C P n}\left[\begin{array}{cc}
0 & -1 \\
1 & 0
\end{array}\right]+\Delta_{M C P}\left[\begin{array}{cc}
0 & -\Delta \omega_{M C P s} \\
\Delta \omega_{M C P s} & 0
\end{array}\right] \\
& =\left[\begin{array}{cc}
-\frac{1}{\tau_{L 0}} & -\left(\Delta \omega_{m 0}+\Delta \omega_{L n}+\Delta \omega_{M C P n}\right) \\
\left(\Delta \omega_{m 0}+\Delta \omega_{L n}+\Delta \omega_{M C P n}\right) & -\frac{1}{\tau_{L 0}}
\end{array}\right] \\
& +\delta_{\beta}\left[\begin{array}{cc}
-\frac{1}{\tau_{L 0}} & -\Delta \omega_{m 0} \\
\Delta \omega_{m 0} & -\frac{1}{\tau_{L 0}}
\end{array}\right]+\Delta_{L F D}\left[\begin{array}{cc}
0 & -\Delta \omega_{L s} \\
\Delta \omega_{L s} & 0
\end{array}\right]+\Delta_{M C P}\left[\begin{array}{cc}
0 & -\Delta \omega_{M C P s} \\
\Delta \omega_{M C P s} & 0
\end{array}\right], \\
& B_{z}\left(\delta_{\beta}\right)=\left[\begin{array}{cc}
\frac{2}{Z_{o}} c_{10} & -\frac{2}{Z_{o}} c_{30} \\
\frac{2}{Z_{o}} c_{30} & \frac{2}{Z_{o}} c_{10}
\end{array}\right]+\delta_{\beta}\left[\begin{array}{cc}
\frac{2}{Z_{o}} c_{10} & -\frac{2}{Z_{o}} c_{30} \\
\frac{2}{Z_{o}} c_{30} & \frac{2}{Z_{o}} c_{10}
\end{array}\right]
\end{aligned}
$$


Let

$$
\begin{aligned}
& \delta_{1}=\delta_{\beta}, \\
& \delta_{2}=\Delta_{L F D}, \\
& \delta_{3}=\Delta_{M C P},
\end{aligned}
$$

and

$$
\begin{aligned}
& A_{z 0}=\left[\begin{array}{cc}
-\frac{1}{\tau_{L 0}} & -\left(\Delta \omega_{m 0}+\Delta \omega_{L n}+\Delta \omega_{M C P n}\right) \\
\left(\Delta \omega_{m 0}+\Delta \omega_{L n}+\Delta \omega_{M C P n}\right) & -\frac{1}{\tau_{L 0}}
\end{array}\right], \\
& A_{z 1}=\left[\begin{array}{cc}
-\frac{1}{\tau_{L 0}} & -\Delta \omega_{m 0} \\
\Delta \omega_{m 0} & -\frac{1}{\tau_{L 0}}
\end{array}\right], \\
& A_{z 2}=\left[\begin{array}{cc}
0 & -\Delta \omega_{L s} \\
\Delta \omega_{L s} & 0
\end{array}\right], \\
& A_{z 3}=\left[\begin{array}{cc}
0 & -\Delta \omega_{M C P s} \\
\Delta \omega_{M C P s} & 0
\end{array}\right], \\
& B_{z 0}=\left[\begin{array}{ll}
\frac{2}{Z_{o}} c_{10} & -\frac{2}{Z_{o}} c_{30} \\
\frac{2}{Z_{o}} c_{30} & \frac{2}{Z_{o}} c_{10}
\end{array}\right], \\
& B_{z 1}=\left[\begin{array}{ll}
\frac{2}{Z_{o}} c_{10} & -\frac{2}{Z_{o}} c_{30} \\
\frac{2}{Z_{o}} c_{30} & \frac{2}{Z_{o}} c_{10}
\end{array}\right],
\end{aligned}
$$

Then,

$$
\begin{aligned}
& A_{z}\left(\Delta w_{L}, \Delta \omega_{m c p}, \delta_{\beta}\right)=A_{z 0}+\sum_{i=1}^{3} \delta_{i} A_{z i}, \\
& B_{z}\left(\delta_{\beta}\right)=B_{z 0}+\sum_{i=1}^{3} \delta_{i} B_{z i} .
\end{aligned}
$$

Since

$$
r_{i}=\operatorname{rank}\left[A_{z i} \quad B_{z i}\right]=2, \quad i=1,2,3,
$$


there exist $E_{z i}, \quad G_{z i}$, and $H_{z i}, i=1,2,3$ such that

$$
\left[\begin{array}{ll}
A_{z i} & B_{z i}
\end{array}\right]=E_{z i}\left[\begin{array}{ll}
G_{z i} & H_{z i}
\end{array}\right], \quad i=1,2,3
$$

where

$$
E_{z i} \in \mathfrak{R}^{2 \times 2}, \quad\left[\begin{array}{ll}
G_{z i} & H_{z i}
\end{array}\right] \in \mathfrak{R}^{2 \times 4} .
$$

Now, define a linear system $G_{S R F}$ with extra inputs and outputs via the state equations

or equivalently via the transfer matrix

$$
\left[\begin{array}{c}
\dot{z} \\
y \\
v_{1} \\
v_{2} \\
v_{3}
\end{array}\right]=\left[\begin{array}{ccccc}
A_{z 0} & B_{z 0} & E_{z 1} & E_{z 2} & E_{z 3} \\
C_{z} & 0 & 0 & 0 & 0 \\
G_{z 1} & H_{z 1} & 0 & 0 & 0 \\
G_{z 2} & H_{z 2} & 0 & 0 & 0 \\
G_{z 3} & H_{z 3} & 0 & 0 & 0
\end{array}\right]\left[\begin{array}{c}
z \\
u \\
w_{1} \\
w_{2} \\
w_{3}
\end{array}\right],
$$

$$
\left[\begin{array}{l}
y \\
v
\end{array}\right]=G_{S R F}\left[\begin{array}{l}
u \\
w
\end{array}\right]=\left[\begin{array}{ll}
G_{S R F 11} & G_{S R F 12} \\
G_{S R F 21} & G_{S R F 22}
\end{array}\right]\left[\begin{array}{l}
u \\
w
\end{array}\right],
$$

where

$$
\begin{aligned}
& v=\left[\begin{array}{lll}
v_{1} & v_{2} & v_{3}
\end{array}\right]^{T}, \\
& w=\left[\begin{array}{lll}
w_{1} & w_{2} & w_{3}
\end{array}\right]^{T}, \\
& G_{S R F}=\left[\begin{array}{ll}
G_{S R F 11} & G_{S R F 12} \\
G_{S R F 21} & G_{S R F 22}
\end{array}\right], \\
& G_{S R F 11} \in C^{2 \times 2}: u \mapsto y, \\
& G_{S R F 12} \in C^{2 \times 6}: w \mapsto y, \\
& G_{S R F 21} \in C^{6 \times 2}: u \mapsto v, \\
& G_{S R F 22} \in C^{6 \times 6}: w \mapsto v
\end{aligned}
$$

as shown in figure 3.4 .

The perturbed system (3.38) and (3.39) is represented via a lower linear fractional transformation (LFT) around $G_{S R F}$, namely,

$$
y=F_{L}\left(G_{S R F}, \Delta_{S R F}\right) u,
$$

where $F_{L}\left(G_{S R F}, \Delta_{S R F}\right)$ is a lower linear fractional transformation (LFT)

$$
F_{L}\left(G_{S R F}, \Delta_{S R F}\right)=G_{S R F 11}+G_{S R F 12} \Delta_{S R F}\left(I-G_{S R F 22} \Delta_{S R F}\right)^{-1} G_{S R F 21}
$$

and $\quad \Delta_{S R F}$ maps $v \mapsto w$ and has the structure given as 


$$
\Delta_{S R F}=\left\{\operatorname{diag}\left[\delta_{1} I_{2}, \delta_{2} I_{2}, \delta_{3} I_{2}\right]: \delta_{i} \in \Re\right\} .
$$

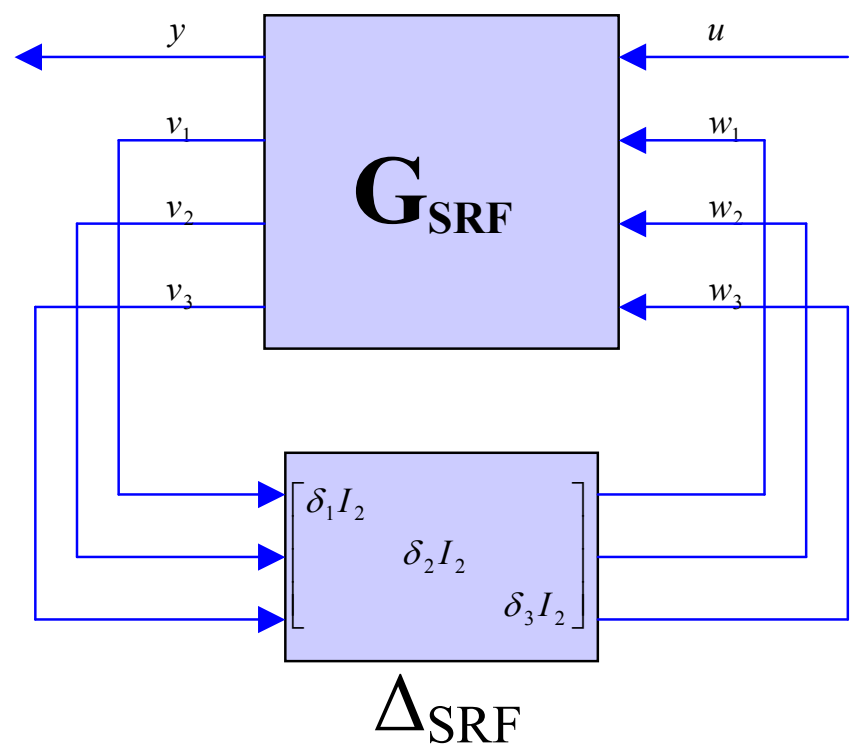

Figure 3.4 Lower LFT of the perturbed SRF cavity model

Since (3.60) is equvalently expressed as

$$
\left[\begin{array}{l}
v \\
y
\end{array}\right]=\left[\begin{array}{ll}
G_{S R F 22} & G_{S R F 21} \\
G_{S R F 12} & G_{S R F 11}
\end{array}\right]\left[\begin{array}{l}
w \\
u
\end{array}\right],
$$

the perturbed system (3.36) and (3.37) is represented via an upper linear fractional transformation (LFT) around $\bar{G}_{S R F}$, namely,

$$
y=F_{U}\left(\bar{G}_{S R F}, \Delta_{S R F}\right) u
$$

where

$$
\bar{G}_{S R F}=\left[\begin{array}{ll}
G_{S R F 22} & G_{S R F 21} \\
G_{S R F 12} & G_{S R F 11}
\end{array}\right]
$$

and $F_{U}\left(\bar{G}_{S R F}, \Delta_{S R F}\right)$ is an upper linear fractional transformation (LFT) 


$$
F_{U}\left(\bar{G}_{S R F}, \Delta_{S R F}\right)=G_{S R F 12} \Delta_{S R F}\left(I-G_{S R F 22} \Delta_{S R F}\right)^{-1} G_{S R F 21}+G_{S R F 11} .
$$

Figure 3.5 shows the upper LFT of the perturbed SRF cavity model.

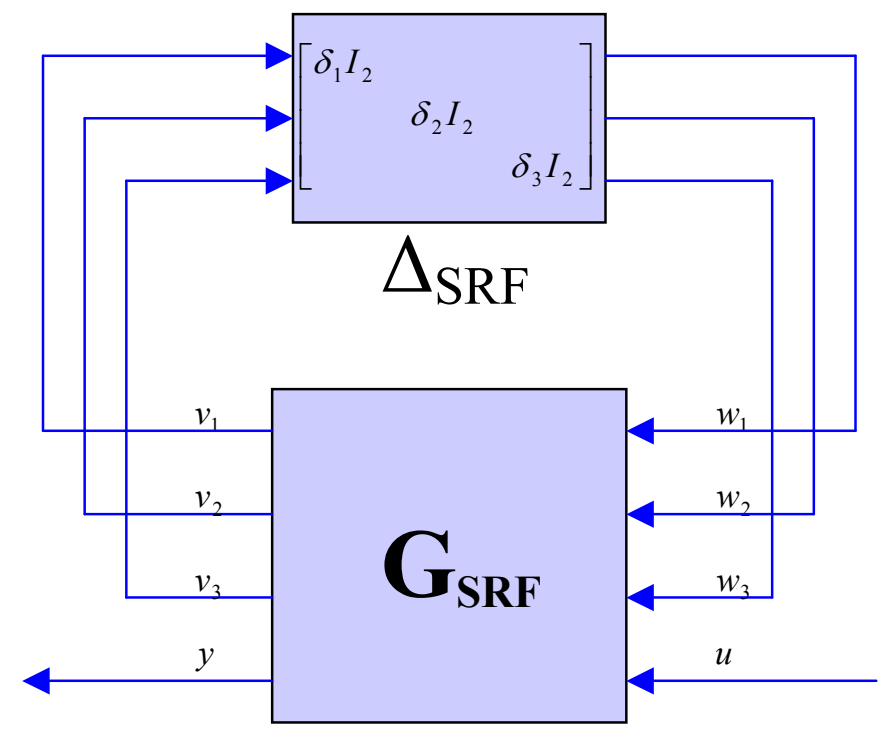

Figure 3.5 Lower LFT of the perturbed SRF cavity model

\section{III-C. NORMAL CONDUCTING CAVITY MODEL}

The normal conducting cavity is similarly modeled as is the SRF cavity. The difference is that for normal conducting cavity, the Lorentz Force Detuning and the microphonics do not apply. The normal conducting cavity can be expressed as the state space model given by

$$
\begin{aligned}
& \dot{z}=A_{c} z+B_{z} u+B_{z I} I \\
& y=C z
\end{aligned}
$$

where

$$
z=\left[\begin{array}{ll}
V_{I} & V_{Q}
\end{array}\right]^{T}, \quad u=\left[\begin{array}{ll}
V_{f I} & V_{f Q}
\end{array}\right]^{T}, \quad I=\left[\begin{array}{ll}
I_{I} & I_{Q}
\end{array}\right]^{T},
$$




$$
\begin{array}{ll}
A_{c}=\left[\begin{array}{cc}
-\frac{1}{\tau_{L}} & -\Delta \omega_{m} \\
\Delta \omega_{m} & -\frac{1}{\tau_{L}}
\end{array}\right], \\
B_{z}=\left[\begin{array}{cc}
\frac{2}{R_{o}} c_{1} & -\frac{2}{R_{o}} c_{3} \\
\frac{2}{R_{o}} c_{3} & \frac{2}{R_{o}} c_{1}
\end{array}\right], \\
C=\left[\begin{array}{ll}
1 & 0 \\
0 & 1
\end{array}\right],
\end{array}
$$

and

$\tau=\frac{2 Q_{o}}{\omega_{o}}:$ Cavity damping time constant $[\mathrm{sec}]$,

$Q_{o}$ : Cavity resonator unloaded quality factor,

$\omega_{o}$ : Cavity resonance frequency $[\mathrm{rad} / \mathrm{sec}]$,

$R_{c u}$ : Resistance of the Equivalent circuit of cavity transformed to RF

generator,

$R_{o}$ : Transmission Line characteristic impedance,

$\Delta \omega_{m}$ : Detuning frequency $[\mathrm{rad} / \mathrm{s}]$,

$V_{f l}, V_{f Q}:$ forward I/Q voltage,

$I_{I}, I_{Q}:$ Beam current in $\mathrm{I} / \mathrm{Q}$,

$V_{I}, V_{Q}:$ Cavity Field in $\mathrm{I} / \mathrm{Q}$,

$\zeta$ : Transformation ratio,

$\tau_{L}=\frac{2 Q_{L}}{\omega_{o}}:$ Loaded cavity damping constant,

$Q_{L}=\frac{Q_{o}}{1+\beta}:$ Loaded $Q$,

$\beta=1+\frac{P_{b}}{P_{c u}}$ : Cavity coupling factor,

$c_{1}=\frac{R_{c u}}{\tau}, \quad c_{3}=\frac{R_{c u}}{2 Q_{o} \tau}$.

The state space model as given in (3.70) and (3.71) can be expressed as the transfer matrix.

$$
Y(s)=G_{c}(s) U(s)+G_{B}(s) I(s)
$$

where

$$
\begin{aligned}
& G_{c}(s)=C_{z}\left(s I-A_{z}\right)^{-1} B_{z}, \\
& G_{B}(s)=C_{z}\left(s I-A_{z}\right)^{-1} B_{z I} .
\end{aligned}
$$

The equation (3.72) shows that from the perspective of a cavity beam current is an exogenous disturbance. Figure 3.6 and figure 3.7 show the frequency responses of 6 DTL tanks and the beam dynamics inside DTL tanks. 
Table 3.2 DTL Tank \#4 parameters, Power control margin $=25 \%$

\begin{tabular}{|c|c|}
\hline$P_{b}, \quad$ Beam Power & $0.576 \mathrm{MW}$ \\
\hline$\phi_{S}, \quad$ Synchronous phase & $-25^{\circ}$ \\
\hline$E_{a c c}, \quad$ Electric Field & $2.958 \mathrm{MV} / \mathrm{m}$ \\
\hline$Q_{o}, \quad$ Unloaded quality factor & 42122 \\
\hline Avrage beam current & $34 m A$ \\
\hline$f_{o}, \quad$ Resonance frequency & $402.5 \mathrm{MHz}$ \\
\hline$\omega_{o}, \quad$ Cavity resonance frequency & $2.529 \mathrm{e}+009 \mathrm{rad} / \mathrm{sec}$ \\
\hline$P_{c u}, \quad$ Cavity wall dissipation & $1.302 \mathrm{MW}$ \\
\hline$P_{g}^{P e a k}=\left(P_{b}+P_{c u}\right) \cdot(1+$ power_control__ $m$ arg in $), \quad$ Generator peak power & $2.3475 \mathrm{MW}$ \\
\hline$R_{O}, \quad$ Transmission line impedance & $50 \Omega$ \\
\hline$V_{\text {gap }}=\frac{P_{b}}{I_{b} \cos \left(\phi_{S}\right)}, \quad$ Peak cavity voltage & $18.703 \mathrm{MV}$ \\
\hline$L_{c a v}=\frac{V_{\text {gap }}}{E_{a c c}}, \quad$ Cavity Length & $6.323 \mathrm{~m}$ \\
\hline$V_{b}=V_{\text {gap }} \cos \left(\phi_{S}\right), \quad$ Beam Voltage & $16.951 M V$ \\
\hline$P_{f}=P_{b}+P_{c u}, \quad$ Forward Power & $1.878 \mathrm{MW}$ \\
\hline$V_{f}=\sqrt{2 R_{o} \cdot P_{f}}, \quad$ Forward Voltage & $13704 \mathrm{~V}$ \\
\hline$I_{f}=\frac{V_{f}}{R_{o}}$, Forward Current & $274.08 \mathrm{~A}$ \\
\hline$\Delta \omega_{m}=\frac{V_{g a p}}{2 P_{c u}} \cdot \mathrm{I}_{\mathrm{b}} \cdot \frac{\omega_{\mathrm{o}}}{\mathrm{Q}_{\mathrm{o}}} \cdot \sin \left(\frac{\pi}{180} \phi_{s}\right), \quad$ Detuning frequency & $-6196.4 \mathrm{rad} / \mathrm{sec}$ \\
\hline$\psi_{m}=-\tan ^{-1}\left(\frac{2 Q_{L} \cdot \Delta \omega_{m}}{\omega_{o}}\right), \quad$ Detuning angle & $4.8307^{\circ}$ \\
\hline$\beta=1+\frac{P_{b}}{P_{c u}}$, Cavity coupling factor & 1.4424 \\
\hline$Q_{L}=\frac{Q_{O}}{1+\beta}$, Loaded quality factor & 17246 \\
\hline$\tau=\frac{2 Q_{o}}{\omega_{o}}, \quad$ Cavity (unloaded) damping time constant & $33.311 \mu \mathrm{sec}$ \\
\hline$\tau_{L}=\frac{2 Q_{L}}{\omega_{o}}$, Loaded time constant & $13.639 \mu \mathrm{sec}$ \\
\hline
\end{tabular}




\begin{tabular}{|l|l|}
\hline$R_{s h}=\frac{V_{\text {gap }}^{2}}{P_{c u}}$, Shunt impedance & $2.6868 \mathrm{e}+008 \Omega$ \\
\hline$R_{c}=\frac{R_{s h}}{2}, \quad$ Cavity resistance & $1.3434 \mathrm{e}+008 \Omega$ \\
\hline$\zeta=\sqrt{\frac{R_{c}}{\beta R_{o}}}, \quad$ Transformer ratio & 1364.8 \\
\hline$R_{c u}=\frac{\mathrm{R}_{\mathrm{c}}}{\zeta}, \quad$ Cavity resistance transformed to the equivalent circuit of $\mathrm{RF}$ & $72.12 \Omega$ \\
\hline$V_{b i}=I_{b} \cdot R_{s h} \cdot \cos (\psi) /(1+\beta), \quad$ Beam induced Voltage & $3.3898 \mathrm{e}+006 \mathrm{~V}$ \\
\hline$P_{g}=P_{f}, \quad$ Generator Power & $1.878 \mathrm{MW}$ \\
\hline$V_{g i}=2 \sqrt{P_{g} \cdot \beta \cdot R_{s h}} \cos (\psi) /(1+\beta)$, Generator induced Voltage & $2.0022 \mathrm{e}+007 \mathrm{~V}$ \\
\hline$I_{g e n}=2 \sqrt{P_{g} \cdot \beta / R_{s h}}$, & $0.20082 \mathrm{~A}$ \\
\hline
\end{tabular}

\begin{tabular}{|l|l|}
\hline$c_{1}=\frac{R_{c u}}{\tau}$ & $2.165 \mathrm{e}+006$ \\
\hline$c_{3}=\frac{R_{c u}}{2 Q_{o} \tau}$ & 25.699 \\
\hline
\end{tabular}



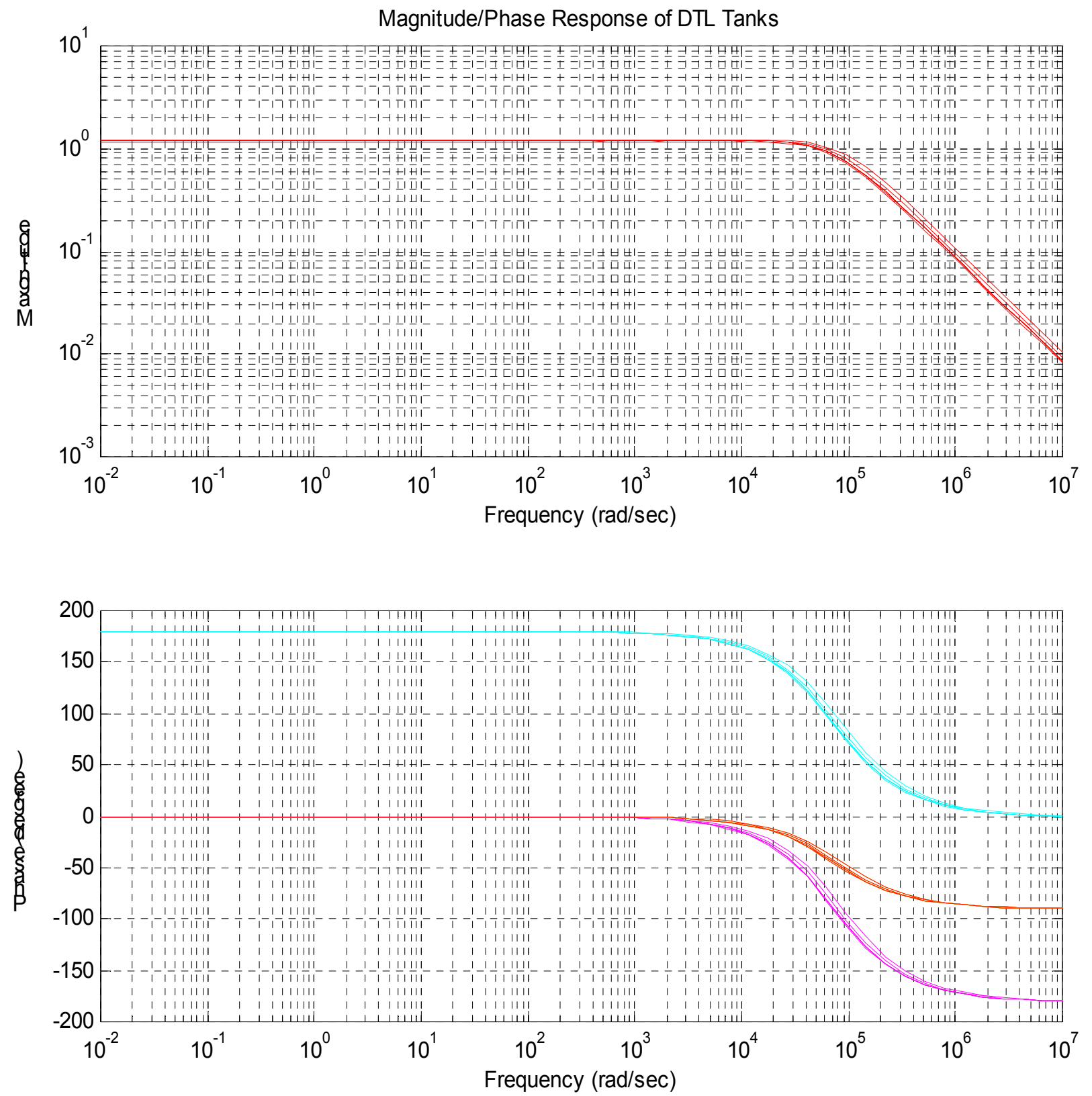

Figure 3.6 Frequency Responses of 6 DTL Tanks, $\left|G_{C}(j \omega)\right|$ and $\angle G_{C}(j \omega)$ 

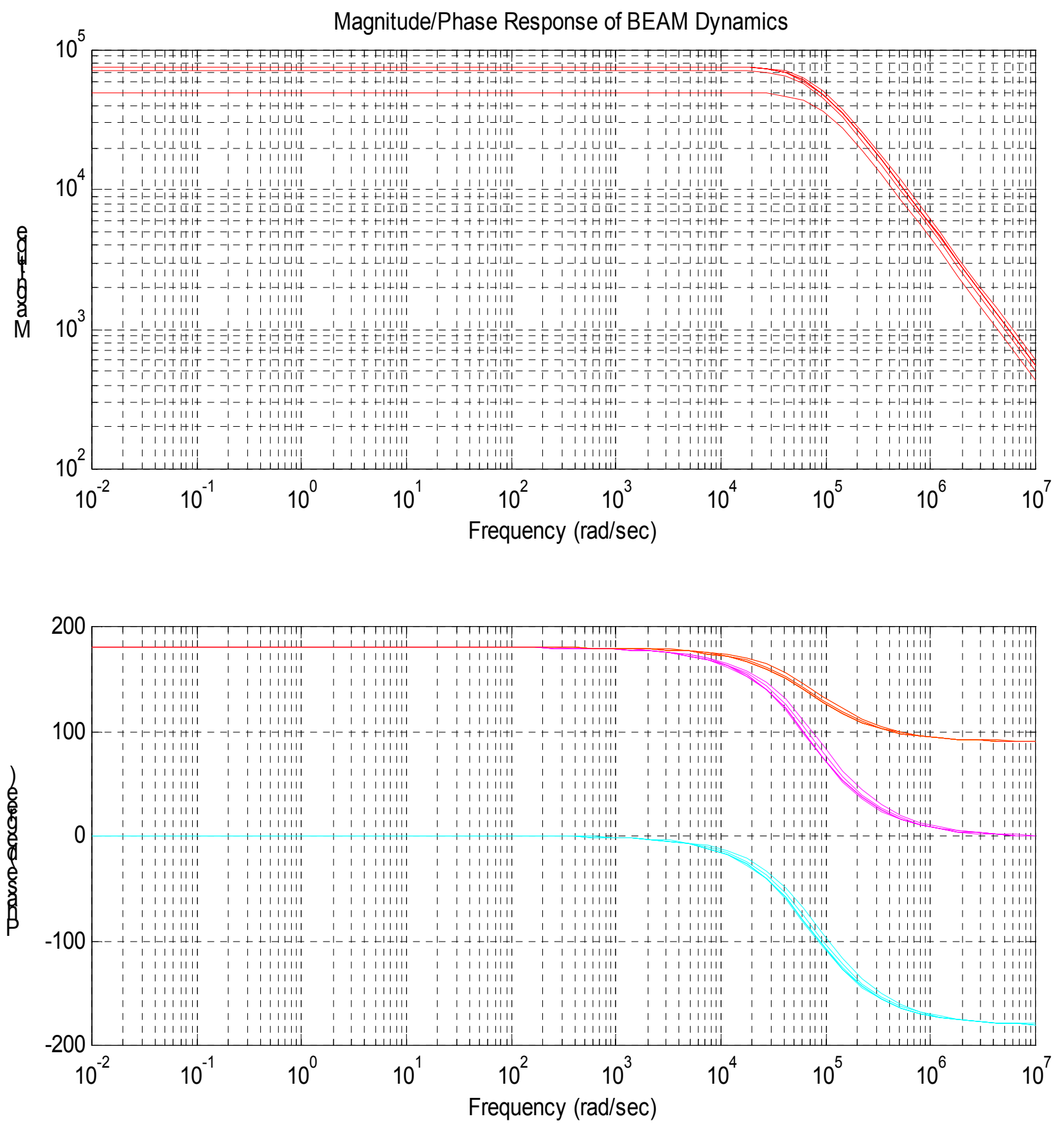

Figure 3.7 Frequency Responses of Beam Dynamics for 6 DTL Tanks, $\left|G_{B}(j \omega)\right|$ and $\angle G_{B}(j \omega)$ 


\section{III-D. SCALING}

In the transfer matrix, the input $u$ and the exogenous disturbance $I$ have different signal levels. The signal level of $u$ is determined by the necessary power of a cavity. The disturbance $I$ has an average of $26 \mathrm{~mA}$ and the peak value is $38 \mathrm{~mA}$ for SNS. In the case of DTL tanks, the range of input is 7127.4 $\mathrm{V} \leq u \leq 13296.6 \mathrm{~V}$ (Table 2.8). Hence, in order to apply the modern system analysis tool such as $\mu$-analysis, the disturbance $I$ is to be scaled.

First, it is easily verified that

$$
B_{z I}=-\zeta R_{o} B_{z}=-B_{z} \zeta R_{o} .
$$

Hence, the transfer matrix is reduced to

$$
Y(s)=G_{c}(s)\left(U(s)-\zeta R_{o} I(s)\right) .
$$

With the average beam current $26 \mathrm{~mA}$, the scaling factor $d_{S I}$ has the range

$$
\frac{26 e-3}{13296.6}=1.9554 \mathrm{e}-006 \leq d_{S I} \leq \frac{26 e-3}{7127.4}=3.6479 \mathrm{e}-006 .
$$

Now, the new variable $\bar{I}$ has the same signal level as the input $u$. Figure 3.7 is the scaled cavity model.

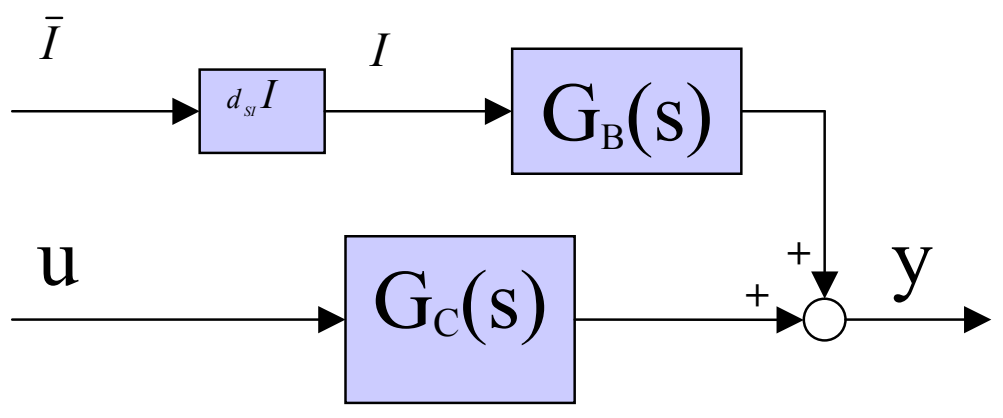

Figure 3.7 Scaled Cavity Model. Beam current $I$ scaled and the new variable $\bar{I}$ is the signal of the same level as the input $u$ 


\section{CABLE DELAY, COMPONENTS UNCERTAINTY}

The analog signals in the cavity are fedback to the digital signal processor for several purposes such as low level RF control signal generation, data display, and data storage. That feedback loop is comprised of RF components such as the RF switch, directional coupler, mixer, I/Q demodulator, preamplifier, bandpass filter, and transformer. Since these components are not perfect, there are amplitude distortions and phase distortions. These distortion are characterized in the frequency domain. Also, there is time delay due to the feedback cable delay and RF components time delay, where significant time delays are yielded from the feedback cable and the FIR filters.

Meanwhile, there exists uncertainties in the forward loop from the digital signal processor output to the klystron. In this forward loop, RF components such as the I/Q modulator, low power amplifier, bandpass filter, medium power amplifier, directional coupler, and switch are placed and these components inevitably generate amplitude distortions and phase distortions.

In this section, amplitude distortions and phase distortions of RF components are modeled. In that model, the amplitude distortions and the phase distortions are modeled as the lumped amplitude distortion and the lumped phase distortion.

\section{IV-A. RF COMPONENTS UNCERTAINTY IN FEEDBACK LOOP , FORWARD LOOP}

The uncertainty in the RF components in the feedback loop is modeled as the multiplicative uncertainty. Figure 4.1 shows the block diagram. In this section, the weighting function matrix $W_{F B}(s)$ and the perturbation $\Delta_{F B}$ will be obtained.

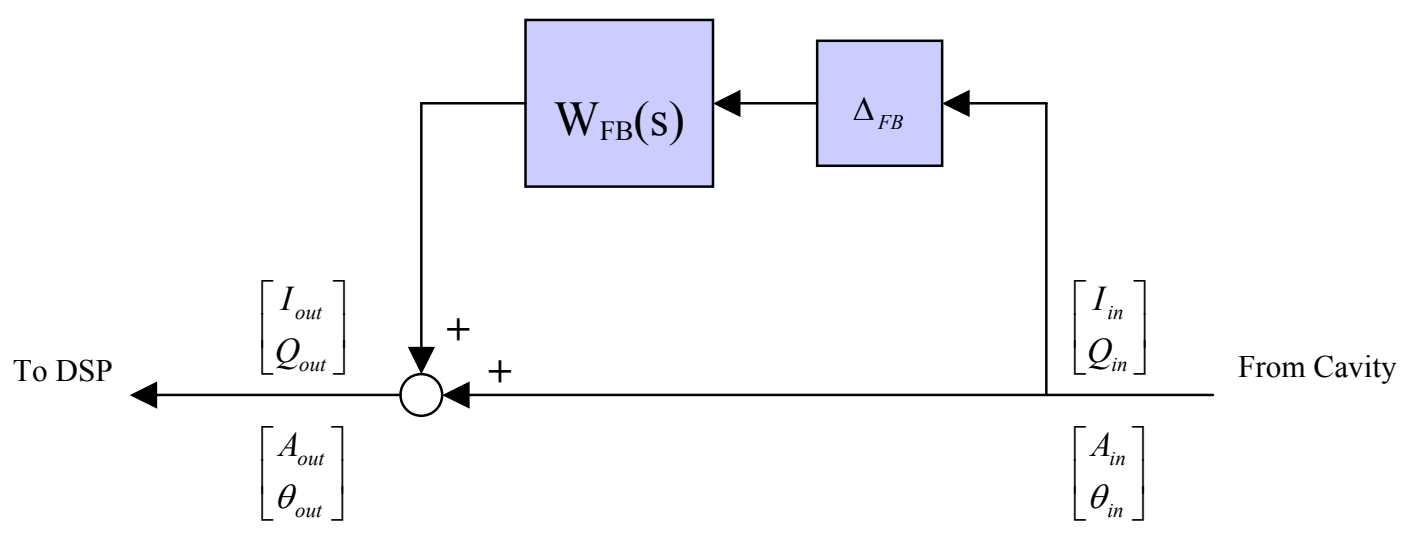

Figure 4.1 Multiplicative Uncertainty Representation of Feedback Loop Distortion 
The amplitude $A_{\text {out }}$ and the phase $\theta_{\text {out }}$ are expressed as

$$
\begin{aligned}
& A_{\text {out }}=\left(1+\Delta_{A F B}\right) A_{\text {in }} \\
& \theta_{\text {out }}=\theta_{\text {in }}+\Delta_{\theta F B}
\end{aligned}
$$

where $\Delta_{A F B}$ is the lumped amplitude distortion in percent and $\Delta_{\theta F B}$ is the lumped phase distortion in radian. In In-Phase (I) and Quadrature (Q) coordinates, the distorted $I_{\text {out }}$ and $Q_{\text {out }}$ are expressed as

$$
\begin{aligned}
I_{\text {out }} & =A_{\text {out }} \cos \left(\theta_{\text {out }}\right) \\
& =\left(1+\Delta_{A F B}\right) A_{\text {in }} \cos \left(\theta_{\text {in }}+\Delta_{\theta F B}\right) \\
& =\left(1+\Delta_{A F B}\right) A_{\text {in }}\left(\cos \left(\theta_{\text {in }}\right) \cos \left(\Delta_{\theta F B}\right)-\sin \left(\theta_{\text {in }}\right) \sin \left(\Delta_{\theta F B}\right)\right) \\
Q_{\text {out }} & =A_{\text {out }} \sin \left(\theta_{\text {out }}\right) \\
& =\left(1+\Delta_{A F B}\right) A_{\text {in }} \sin \left(\theta_{\text {in }}+\Delta_{\text {OFB }}\right) \\
& \left.=\left(1+\Delta_{A F B}\right) A_{\text {in }}\left(\sin \left(\theta_{\text {in }}\right) \cos \left(\Delta_{\theta F B}\right)+\cos \theta_{\text {in }}\right) \sin \left(\Delta_{\theta F B}\right)\right)
\end{aligned}
$$

In a compact matrix form, $I_{\text {out }}$ and $Q_{\text {out }}$ are expressed as

$$
\begin{aligned}
{\left[\begin{array}{l}
I_{\text {out }} \\
Q_{\text {out }}
\end{array}\right]=} & \left(1+\Delta_{A F B}\right) A_{\text {in }}\left[\begin{array}{cc}
\cos \left(\Delta_{\theta F B}\right) & -\sin \left(\Delta_{\theta F B}\right) \\
\sin \left(\Delta_{\theta F B}\right) & \cos \left(\Delta_{\theta F B}\right)
\end{array}\right]\left[\begin{array}{c}
\cos \left(\theta_{\text {in }}\right) \\
\sin \left(\theta_{\text {in }}\right)
\end{array}\right] \\
& =\left(1+\Delta_{A F B}\right)\left[\begin{array}{cc}
\cos \left(\Delta_{\theta F B}\right) & -\sin \left(\Delta_{\theta F B}\right) \\
\sin \left(\Delta_{\theta F B}\right) & \cos \left(\Delta_{\theta F B}\right)
\end{array}\right] A_{\text {in }}\left[\begin{array}{c}
\cos \left(\theta_{\text {in }}\right) \\
\sin \left(\theta_{\text {in }}\right)
\end{array}\right] \\
& =\left(1+\Delta_{A F B}\right)\left[\begin{array}{cc}
\cos \left(\Delta_{\theta F B}\right) & -\sin \left(\Delta_{\theta F B}\right) \\
\sin \left(\Delta_{\theta F B}\right) & \cos \left(\Delta_{\theta F B}\right)
\end{array}\right]\left[\begin{array}{l}
I_{\text {in }} \\
Q_{\text {in }}
\end{array}\right] .
\end{aligned}
$$

It is possible to represent (4.3) in the form of a multiplicative uncertainty as

$$
\left[\begin{array}{c}
I_{\text {out }}(s) \\
Q_{\text {out }}(s)
\end{array}\right]=\left(I+W_{F B}(s) \Delta_{F B}(s)\right)\left[\begin{array}{c}
I_{\text {in }}(s) \\
Q_{\text {in }}(s)
\end{array}\right] .
$$

By Tayor series expansion, the perturbed term in (4.3) is reduced to

$$
\begin{aligned}
\left(1+\Delta_{A F B}\right)\left[\begin{array}{cc}
\cos \left(\Delta_{\theta F B}\right) & -\sin \left(\Delta_{\theta F B}\right) \\
\sin \left(\Delta_{\theta F B}\right) & \cos \left(\Delta_{\theta F B}\right)
\end{array}\right] \approx\left(1+\Delta_{A F B}\right)\left[\begin{array}{cc}
1-\frac{1}{2} \Delta_{\theta F B}^{2} & -\Delta_{\theta F B} \\
\Delta_{\theta F B} & 1-\frac{1}{2} \Delta_{\theta F B}^{2}
\end{array}\right] \\
=\left(1+\Delta_{A F B}\right)\left(\left[\begin{array}{cc}
1 & 0 \\
0 & 1
\end{array}\right]+\left[\begin{array}{cc}
0 & -\Delta_{\theta F B} \\
\Delta_{\theta F B} & 0
\end{array}\right]+\left[\begin{array}{cc}
-\frac{1}{2} \Delta_{\theta F B}^{2} & 0 \\
0 & -\frac{1}{2} \Delta_{\theta F B}^{2}
\end{array}\right]\right) .
\end{aligned}
$$


Define

$$
\begin{aligned}
\bar{\Delta}_{F B}\left(\Delta_{A F B}, \Delta_{\theta F B}\right)= & {\left[\begin{array}{cc}
\Delta_{A F B} & -\Delta_{\theta F B} \\
\Delta_{\theta F B} & \Delta_{A F B}
\end{array}\right]+\Delta_{A F B}\left[\begin{array}{cc}
0 & -\Delta_{\theta F B} \\
\Delta_{\theta F B} & 0
\end{array}\right]+\left[\begin{array}{cc}
-\frac{1}{2} \Delta_{\theta F B}^{2} & 0 \\
0 & -\frac{1}{2} \Delta_{\theta F B}^{2}
\end{array}\right] } \\
& +\Delta_{A F B}\left[\begin{array}{cc}
-\frac{1}{2} \Delta_{\theta F B}^{2} & 0 \\
0 & -\frac{1}{2} \Delta_{\theta F B}^{2}
\end{array}\right]
\end{aligned}
$$

Then, (4.3) is reduced to

$$
\left[\begin{array}{l}
I_{\text {out }} \\
Q_{\text {out }}
\end{array}\right]=\left(I+\bar{\Delta}_{F B}\left(\Delta_{A F B}, \Delta_{\theta F B}\right)\right)\left[\begin{array}{l}
I_{\text {in }} \\
Q_{\text {in }}
\end{array}\right]
$$

In order to represent the perturbed system (4.6) in the standard form of uncertainty as given in (4.4), $\bar{\Delta}_{F B}\left(\Delta_{A F B}, \Delta_{\theta F B}\right)$ is described as

$$
\bar{\Delta}_{F B}\left(\Delta_{A F B}, \Delta_{\theta F B}\right)=W_{F B}(s) \Delta_{F B}\left(\Delta_{A F B}, \Delta_{\theta F B}\right)
$$

where

$$
\left\|\Delta_{F B}\left(\Delta_{A F B}, \Delta_{\theta F B}\right)\right\|_{\infty} \leq 1, \quad \forall \omega
$$

and the weighting function matrix $W_{F B}(s)$ is determined so that it reflects all frequency components that are in $\bar{\Delta}_{F B}\left(\Delta_{A F B}, \Delta_{\theta F B}\right)$.

A way to obtain (4.7) is as follow.

1. For $\min \left(\Delta_{A F B}\right) \leq \Delta_{A F B} \leq \max \left(\Delta_{A F B}\right), \quad \min \left(\Delta_{\theta F B}\right) \leq \Delta_{\theta F B} \leq \max \left(\Delta_{\theta F B}\right)$, obtain the $\bar{\Delta}_{F B}\left(\Delta_{A F B}, \Delta_{\theta F B}\right)$.

2. Obtain

$$
l_{F B}=\max \left|\bar{\Delta}_{F B}\left(\Delta_{A F B}, \Delta_{\theta F B}\right)\right| .
$$

3. $W_{F B}(s)$ is determined such that

$$
\begin{aligned}
& W_{F B}(s)=\left[\begin{array}{cc}
w_{F B}(s) & 0 \\
0 & w_{F B}(s)
\end{array}\right] \\
& W_{F B}(j \omega) \geq l_{F B}, \quad \forall \omega .
\end{aligned}
$$


Then, the standard multiplicative uncertainty form for $\left[\begin{array}{l}I_{\text {out }} \\ Q_{\text {out }}\end{array}\right]$ is obtained.

$$
\left[\begin{array}{l}
I_{\text {out }}(s) \\
Q_{\text {out }}(s)
\end{array}\right]=\left(I+W_{F B}(s) \Delta_{F B}\left(\Delta_{A F B}, \Delta_{\theta F B}\right)\right)\left[\begin{array}{l}
I_{\text {in }}(s) \\
Q_{\text {in }}(s)
\end{array}\right] .
$$

In a similar way, the standard multiplicative uncertainty form for the uncertainties in the forward loop can be obtained. Define

$$
\begin{aligned}
\bar{\Delta}_{F O R}\left(\Delta_{A F O R}, \Delta_{\theta F O R}\right) & =\left[\begin{array}{cc}
\Delta_{A F O R} & -\Delta_{\theta F O R} \\
\Delta_{\theta F O R} & \Delta_{A F O R}
\end{array}\right]+\Delta_{A F O R}\left[\begin{array}{cc}
0 & -\Delta_{\theta F O R} \\
\Delta_{\theta F O R} & 0
\end{array}\right]+\left[\begin{array}{cc}
-\frac{1}{2} \Delta_{\theta F O R}^{2} & 0 \\
0 & -\frac{1}{2} \Delta_{\theta F O R}^{2}
\end{array}\right] \\
& +\Delta_{A F O R}\left[\begin{array}{cc}
-\frac{1}{2} \Delta_{\theta F O R}^{2} & 0 \\
0 & -\frac{1}{2} \Delta_{\theta F O R}^{2}
\end{array}\right]
\end{aligned}
$$

where $\Delta_{A F O R}$ is the lumped amplitude distortion in percent and $\Delta_{\theta F O R}$ is the lumped phase distortion in radians. Then,

$$
\left[\begin{array}{c}
I_{\text {out }}(s) \\
Q_{\text {out }}(s)
\end{array}\right]=\left(I+W_{F O R}(s) \Delta_{F O R}\left(\Delta_{A F O R}, \Delta_{\theta F O R}\right)\right)\left[\begin{array}{c}
I_{\text {in }}(s) \\
Q_{\text {in }}(s)
\end{array}\right] .
$$

where

$$
\left\|\Delta_{F O R}\left(\Delta_{A F O R}, \Delta_{\theta F O R}\right)\right\|_{\infty} \leq 1, \quad \forall \omega
$$

and the weighting function matrix $W_{F O R}(s)$ is the solution of the following procedure.

1. For $\min \left(\Delta_{A F O R}\right) \leq \Delta_{A F O R} \leq \max \left(\Delta_{A F O R}\right)$, and $\min \left(\Delta_{\theta F O R}\right) \leq \Delta_{\theta F O R} \leq \max \left(\Delta_{\theta F O R}\right)$, obtain the $\bar{\Delta}_{F O R}\left(\Delta_{A F O R}, \Delta_{\theta F O R}\right)$.

2. Obtain

$$
l_{F O R}=\max \left|\bar{\Delta}_{F O R}\left(\Delta_{A F O R}, \Delta_{\theta F O R}\right)\right|
$$

3. $W_{F O R}(s)$ is determined such that

$$
\begin{aligned}
& W_{F O R}(s)=\left[\begin{array}{cc}
w_{F O R}(s) & 0 \\
0 & w_{F O R}(s)
\end{array}\right] \\
& W_{F O R}(j \omega) \geq l_{F O R}, \quad \forall \omega .
\end{aligned}
$$

Figure 4.2 shows the standard multiplicative uncertainty form for the forward loop distortion. 


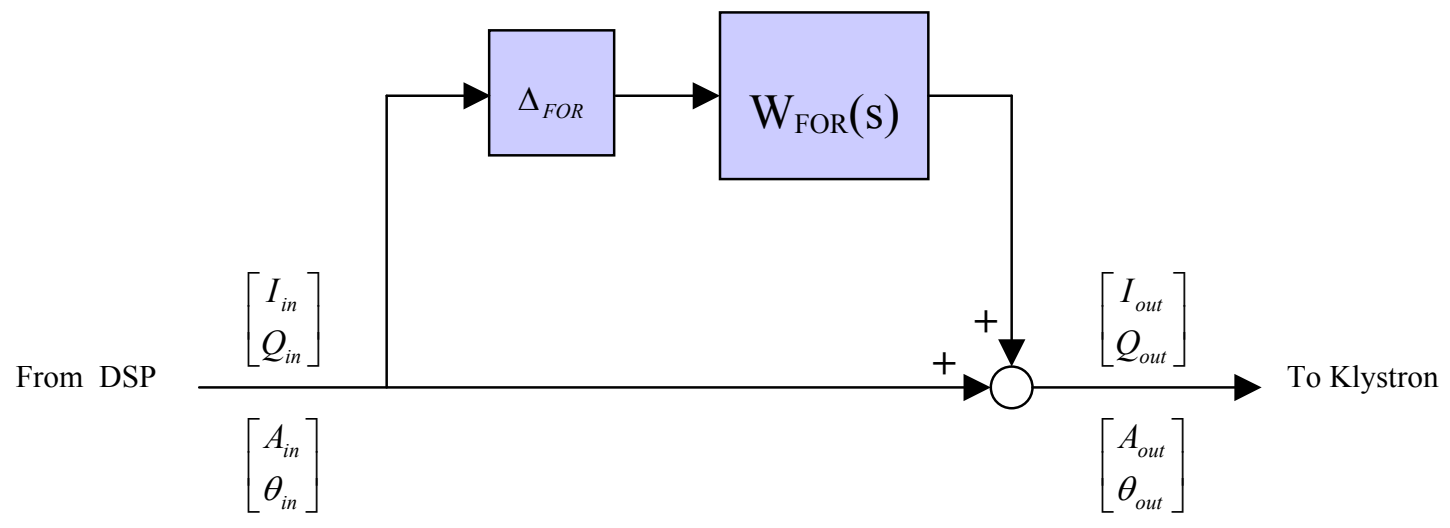

Figure 4.2 Multiplicative Uncertainty Representation of Forward Loop Distortion

\section{IV-B. TIME DELAY MODEL}

Now, consider a system that has a time delay $\tau_{d}$ (i.e., $\left.e^{-\tau_{s} s}\right)$. For a nominal system $G_{o}(s)$, time delay can be integrated into the nominal system as the form

$$
G_{P}(s)=G_{o}(s) e^{-\tau_{s} s}
$$

Hence, time delay can be modeled as an input multiplicative uncertainty.

$$
\left[\begin{array}{c}
I_{\text {out }}(s) \\
Q_{\text {out }}(s)
\end{array}\right]=\left(I+W_{\text {td }}(s) \Delta_{\text {zd }}\right)\left[\begin{array}{l}
I_{\text {in }}(s) \\
Q_{\text {in }}(s)
\end{array}\right]
$$

Figure 4.3 shows the standard multiplicative uncertainty form for the lumped time delay.

Consider the maximum delay, $\tau_{d \max }$, for which the relative error with respect to the delay-free system is $\left|1-\exp \left(-j \omega \tau_{d \max }\right)\right|$. The amplitude $\left|1-\exp \left(-j \omega \tau_{d \max }\right)\right|$ is shown in figure 4.4. The relative uncertainty due to the maximum time delay crosses 1.0 in magnitude at about frequency $1 / \tau_{d \max }$, reaches 2.0 at frequency $\pi / \tau_{d \max }$ where $\exp \left(-j \omega \tau_{d \max }\right)=-1.0$, and oscillates at higher frequencies with maximum 
value 2.0. It follows that if all time delay $\tau_{d}, 0 \leq \tau_{d} \leq \tau_{d \max }$, is considered, then the relative error bound is 2.0 at frequencies above $\pi / \tau_{d \max }$. Hence,

$$
l_{\tau d}(j \omega)=\left\{\begin{array}{cc}
\left|1-\exp \left(-j \omega \tau_{d \max }\right)\right|, & \omega<\pi / \tau_{d \max } \\
2.0, & \omega \geq \pi / \tau_{d \max }
\end{array}\right.
$$

The weight function $W_{\tau d}(s)$ for the time delay $e^{-\tau_{s} s}$ is determined satisfying

$$
\left|W_{\tau d}(j \omega)\right| \geq l_{\tau d}(j \omega), \quad \forall \omega
$$

Widely used weighting function $W_{\tau d}(s)$ for the time delay $e^{-\tau_{s} s}$ is

$$
\begin{aligned}
& W_{\tau d}(s)=\left[\begin{array}{cc}
w_{\tau d}(s) & 0 \\
0 & w_{\tau d}(s)
\end{array}\right] \\
& w_{\tau d}(s)=\frac{\tau d_{\max } s}{\frac{\tau d_{\max }}{2} s+1} .
\end{aligned}
$$

In the current model of the RF system for SNS, the time delays in the loop are

$$
\begin{array}{ll}
\tau_{K l y s t r o n}=1.5 \mathrm{e}-7 \mathrm{sec} & : \text { time delay inside the klystron, } \\
\tau_{W G}=1.21 \mathrm{e}-7 \mathrm{sec} & : \text { time delay in the waveguide transmission }(100 \mathrm{ft}), \\
\tau_{F B C}=1.21 \mathrm{e}-7 \mathrm{sec} & : \text { time delay in the field signal feedback cable }(100 \mathrm{ft}), \\
\tau_{D S P}=1.0 \mathrm{e}-6 \mathrm{sec} & : \text { time delay for the signal processing in the DSP and } \\
& \text { interface. }
\end{array}
$$

Hence, the total time delay $\tau_{d}$ is

$$
\tau_{d}=\tau_{K l y s t r o n}+\tau_{W G}+\tau_{F B C}+\tau_{D S P}=1.392 \mu \mathrm{sec}
$$

and the weighting function $W_{x d}(s)$ is

$$
W_{\tau d}(s)=\frac{1.392 e-006 s}{\frac{1.392 e-006}{2} s+1}
$$

The frequency response of $W_{\tau d}(s)$ is shown in figure 4.4. Note that the more time delay is allowable. However, in that situation, the closed loop system bandwidth should be reduced, which results in the sacrifice of tracking performance, disturbance rejection of the closed loop system. 

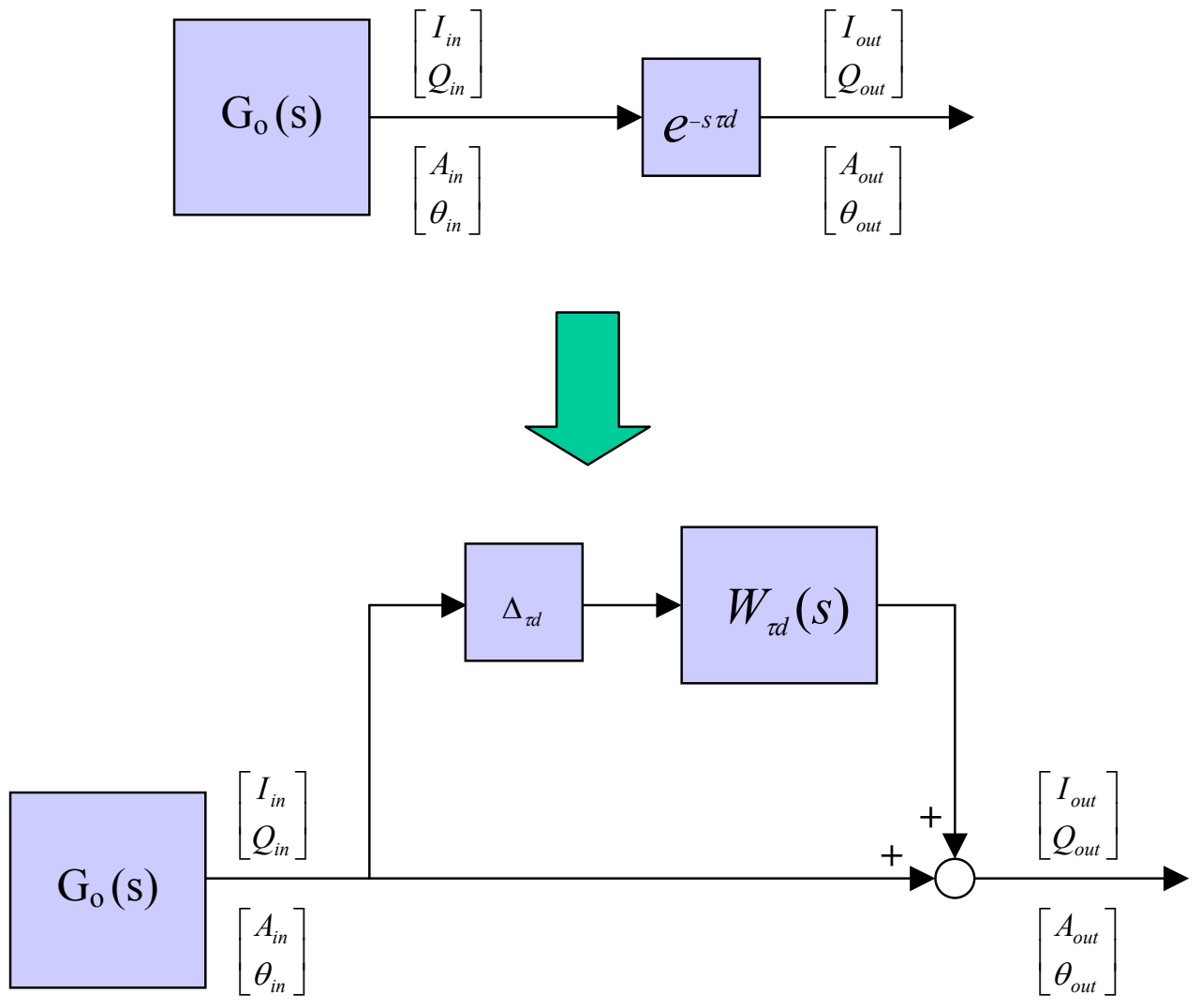

Figure 4.3 Input multiplicative uncertainty representation for the time delay in the loop 


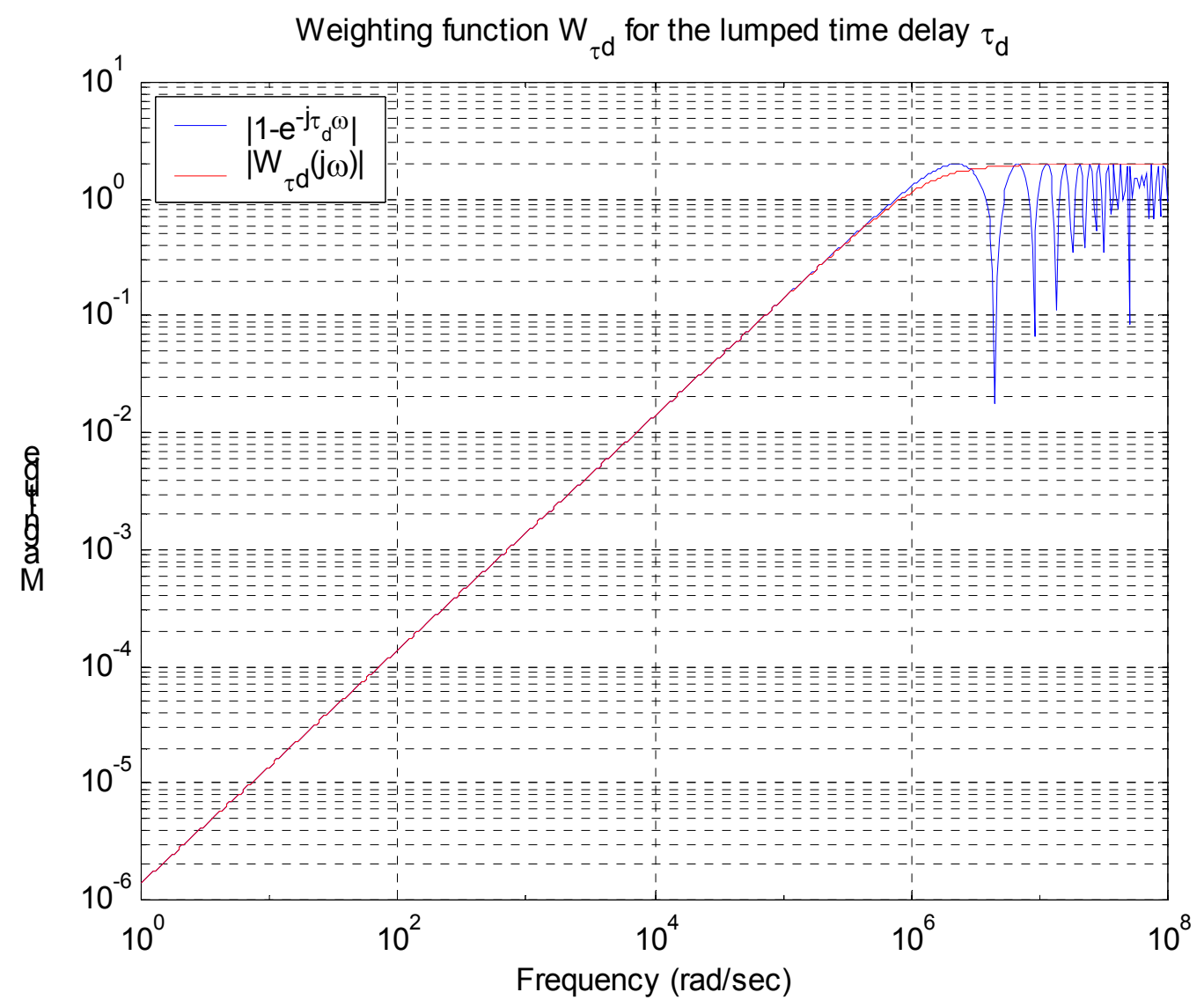

Figure 4.4 Frequency responses of the relative uncertainty $1-e^{-\tau_{d} s}$ for the time delay $\tau_{d}$ and weighting function $W_{\tau d}(s)$ 


\section{LINEAR FRACTIONAL TRANSFORMATION (LFT) REPRESENTATION OF A PERTURBED MODEL OF LINEAR ACCELERATOR RF SYSTEM}

In section II, the klystron normalization and the klystron state space model is addressed. Also, the effect of HVPS ripple on the klystron output is modeled as a output multiplicative uncertainty and as a exogenous disturbance. The nominal klystron model is given by (2.36) and (2.37). The perturbed klystron model due to HVPS ripple is given by (2.67) and (2.68). Representation of the perturbed klystron model has two options; the first one is multiplicative uncertainty representation of the HVPS ripple effect, which is given by the transfer matrix (2.69) and (2.70) and shown in figure 2.25. The second is the exogenous representation of the HVPS ripple, which is given by the transfer matrices (2.71) and (2.72), and shown in figure 2.26. The exogenous disturbance representation of the HVPS ripple is an alternative and can indicate the effect of the HVPS ripple on the klystron output directly. Hence, the latter model is taken for the analysis and synthesis investigated in the following sections.

In section III, the SRF cavity and the normal conducting cavity have been modeled. Also, the perturbation on the cavity parameters such as Q has been investigated and the perturbed cavity is modeled. The SRF cavity model is givn by (3.7) and (3.8) and the normal conducting cavity model is given by (3.69) and (3.70). The transfer matrix representation of the SRF cavity is given by (3.12) and the transfer matrix representation of the normal conducting cavity is given by (3.71). From the perspective of SRF cavity or normal conducting cavity, the beam current is an exogenous disturbance. This relation is shown in block diagram figure 3.1. In the SRF cavity, the system matrix contains the Lorentz Force Detuning term, whereas in the normal conducting cavity model, it is not there. For a SRF cavity, when there are parameter perturbations as well as the Lorentz Force Detuning and microphonics, then the cavity model is replaced with the lower LFT (3.61), or the upper LFT (3.66) and the corresponding block diagram figure 3.4 or figure 3.5 , respectively.

In section IV, the uncertainties in the RF components and signal processing interface have been modeled. The uncertainties in the feedback loop and in the forward loop have been modeled as multiplicative uncertainties. Equation (4.10) is the model of the feedback loop multiplicative uncertainty and (4.12) is the model of the forward multiplicative uncertainty. Figure 4.1 and figure 4.2 are their block diagrams. Also, the times delays in the loops has been lumped and modeled as a multiplicative uncertainty. Equation (4.16) is the model for the time delay and figure 4.3 is the block diagram of the multiplicative uncertainty.

In this section, all the models are integrated and a perturbed linear accelerator RF system model is derived. The importance of scaling to be considered when subsystems are integrated is studied everywhere. When klystron, cavity, disturbances, and other uncertainties are merged into one perturbed system, the klystron input is treated as the reference for scaling. Namely, the klystron input represented by $u$, is scaled in such a way that

$$
\|u\|_{2} \leq 1.0
$$

and all weighting function matrices are determined so that the inputs to these weighting function matrices are scaled and their norms are less than or equal to 1.0. The scaling of the klystron input yields a gain matrix, which may be lumped into the klystron gain.

Figure 5.1 shows the perturbed linear accelerator RF system model. The system has three exogenous siganls. $\tilde{d}_{R},\left\|\tilde{d}_{R}\right\|_{2} \leq 1.0$, is the disturbance representing HVPS ripple, $\tilde{d}_{B},\left\|\tilde{d}_{B}\right\|_{2} \leq 1.0$, is the scaled disturbance representing beam current. The weighting function matrix $W_{\text {Ripple }}$ is the lumped one of two blocks shown in figure 2.26. That is, 


$$
W_{\text {Ripple }}(s) \quad \Leftarrow \quad G(s) W_{\text {Ripple }}(s)
$$

Also, the weighting function matrix $W_{B E A M}$ for $\widetilde{d}_{B}$ is the lumped transfer matrix of $G_{B}(s)$ shown in figure 3.1 and $\operatorname{diag}\left(\frac{1}{\left|I_{I}\right|}, \frac{1}{\left|I_{Q}\right|}\right)$ where $I_{I}=I \cos \left(\phi_{B E A M}\right), I_{I}=I \sin \left(\phi_{B E A M}\right)$, and $I$ is the average beam current and $\phi_{B E A M}$ is the beam angle. That is,

$$
W_{B E A M}(s) \quad \Leftarrow \quad G_{B}(s)\left[\begin{array}{cc}
\frac{1}{\left|I_{I}\right|} & 0 \\
0 & \frac{1}{\left|I_{Q}\right|}
\end{array}\right]
$$

The reference signal $r$ is such that

$$
\|r\|_{2} \leq 1.0
$$

When the reference $r$ is required to be $\|r\|_{2}>1.0$, then a weighting function matrix $W_{r}(s)$ is inserted so that the scaled reference $\widetilde{r}$ to the error calculation block satisfies $\|\widetilde{r}\|_{2} \leq 1.0$.

In figure 5.1, there is a weighting function matrix $W_{\text {perf }}(s)$. This weighting function matrix specifies the closed loop system performance. The typical specifications of the closed loop system include:

1. Minimum bandwidth frequency $\omega_{B}$ (defined as the frequency where the maximum singular value of the sensitivity matrix $S$ crosses $\frac{1}{\sqrt{2}}$ from below).

2. Maximum tracking error at selected frequencies.

3. System type, or alternatively, the maximum steady-state tracking error, $A$.

4. Shape of $\mathrm{S}$ over the selected frequency range.

5. Maximum peak magnitude of $S,\|S(j \omega)\|_{\infty} \leq M$.

Mathematically, these specifications may be captured by a weighting function matrix $W_{\text {perf }}(s)$ which is specified by the designer. The performance requirement becomes

$$
\left\|W_{\text {perf }} S\right\|_{\infty}<1
$$

Usual selection for the performance weighting function $W_{\text {perf }}(s)$ is

$$
\begin{aligned}
& W_{\text {perf }}(s)=\left[\begin{array}{cc}
w_{\text {perf }}(s) & 0 \\
0 & w_{\text {perf }}(s)
\end{array}\right] \\
& w_{\text {perf }}(s)=\frac{s / M+\omega_{B}}{s+A \omega_{B}} .
\end{aligned}
$$

In the linear accelerator RF system of SNS, the performance specification for the closed loop system is 


$$
\begin{aligned}
& \text { The maximum steady-state tracking errors : } \begin{array}{l}
0.5 \% \text { in amplitude } \\
0.5 \text { degree in phase. }
\end{array} \\
& \text { Shape of } S \text { over selected frequency } \omega_{B} \cdot \\
& \text { Maximum peak magnitude of } \mathrm{S},\|S(j \omega)\|_{\infty} \leq 2 .
\end{aligned}
$$

When the closed loop system bandwidth $\omega_{B}$ is to be $\omega_{B}^{*}$, the performance weighting function $w_{\text {perf }}(s)$ is

$$
w_{\text {perf }}(s)=\frac{s / 2+\omega_{B}^{*}}{s+0.005 \cdot \omega_{B}^{*}} .
$$

Note that as mentioned in the previous section, the maximum of the closed loop system bandwidth $\omega_{B}$ is restricted by the time delays in the system.

From figure 5.1, a generalized closed loop system in the context of the $H_{\infty}$ control theory is obtained. The generalized closed loop system is represented by the Linear Fractional Transformation (LFT). The blocks whose boundaries are dashed-line in figure 5.2 are the uncertainty blocks. They have the inputs $z_{x x x}$ which are the outputs from the system and outputs $w_{y y y}$ which are the inputs to the weighting function matrices. There are other inputs such as exogenous disturbances $\tilde{d}_{R}, \tilde{d}_{B}$ and the set point trajectory $r$ and the control input $u_{C}$. There are other outputs such as the performance weighting function matrix output $\widetilde{e}^{k}$, plant output (cavity field) $y$, tracking error $e^{k}$. In figure 5.2, the outputs are the outward signals from the blue dashed block and the inputs are the inward signals to the blue dashed block. Grouping the inputs in the order of inputs to the weighting function matrix blocks, exogenous disturbances, set point trajectory, and control input, results in

$$
\left\lfloor\begin{array}{llllllllll}
w_{F O R} & w_{\tau d} & w_{F B} & : & \tilde{d}_{R} & \tilde{d}_{B} & : & r & : & u_{C}
\end{array}\right\rfloor
$$

Grouping the outputs in the order of the inputs to uncertainty blocks, plant output (cavity field) $y$, as the performance weighting function matrix output $\tilde{e}^{k}$, tracking error $e^{k}$, results in

$$
\left[\begin{array}{llllllll}
z_{F O R} & z_{\tau d} & z_{F B} & : & y & \tilde{e}^{k} & : & e^{k}
\end{array}\right]
$$

Then, the open loop generalized system is given by 


$$
\left[\begin{array}{c}
z_{F O R} \\
z_{\tau d} \\
z_{F B} \\
\cdots \\
y \\
\tilde{e}^{k} \\
\cdots \\
e^{k}
\end{array}\right]=G_{P}\left[\begin{array}{c}
w_{F O R} \\
w_{\tau d} \\
w_{F B} \\
\cdots \\
\tilde{d}_{R} \\
\tilde{d}_{B} \\
r \\
\cdots \\
u_{C}
\end{array}\right]=\left[\begin{array}{ccccc}
G_{11} & \vdots & G_{12} & \vdots & G_{13} \\
\cdots & \ldots & \cdots & \cdots & \cdots \\
G_{21} & \vdots & G_{22} & \vdots & G_{23} \\
\ldots & \ldots & \cdots & \ldots & \cdots \\
G_{31} & \vdots & G_{32} & \vdots & G_{33}
\end{array}\right]\left[\begin{array}{c}
w_{F O R} \\
w_{\tau d} \\
w_{F B} \\
\cdots \\
\tilde{d}_{R} \\
\tilde{d}_{B} \\
r \\
\cdots \\
u_{C}
\end{array}\right]
$$

where $G_{i j}, i, j=1,2,3$, are transfer matrices with appropriate dimensions.

Figure 5.3 shows the open loop generalized system.

Also, a controller $C$ is connected between tracking error $e^{k}$ and control input $u_{C}$, and then, the closed loop generalized system is obtained as shown in figure 5.4.

$$
\left[\begin{array}{c}
z_{F O R} \\
z_{\tau d} \\
z_{F B} \\
\cdots \\
y \\
\tilde{e}^{k}
\end{array}\right]=G_{P}\left[\begin{array}{c}
w_{F O R} \\
w_{\tau d} \\
w_{F B} \\
\cdots \\
\tilde{d}_{R} \\
\widetilde{d}_{B} \\
r
\end{array}\right]=\left[\begin{array}{ccccc}
G_{C L P 11} & \vdots & G_{C L P 12} & \vdots & G_{C L P 13} \\
\cdots & \cdots & \cdots & \cdots & \cdots \\
G_{C L P 21} & \vdots & G_{C L P 22} & \vdots & G_{C L P 23}
\end{array}\right]\left[\begin{array}{c}
w_{F O R} \\
w_{\tau d} \\
w_{F B} \\
\cdots \\
\widetilde{d}_{R} \\
\widetilde{d}_{B} \\
r
\end{array}\right]
$$

where $G_{C L P i j}, i=1,2, j=1,2,3$, are transfer matrices with appropriate dimensions. In figure 5.4, the uncertainty blocks are connected properly so that they are reflecting the input-output relations on the uncertainty blocks in the closed loop system configuration as shown in figure 5.1.

In order to investigate the open loop system characteristics against the specific signals whatever they are (uncertainties, disturbance, set point trajectory), in figure 5.3, the specific signal is set nonzero and other signals are set zero and then, the outputs are taken into consideration.

The nominal performance problem is investigated in the configuration shown in figure 5.4 where the uncertainty blocks are zero and the exogenous disturbances are zero.

The robust stability problem is investigated in the configuration shown in figure 5.4 where the exogenous disturbances are zero and the robust stability is checked for the uncertainty block

$$
\left[\begin{array}{lll}
\Delta_{F B} & & \\
& \Delta_{\tau d} & \\
& & \Delta_{F O R}
\end{array}\right] .
$$


The robust performance problem is investigated in the configuration shown in figure 5.5 where the uncertainty blocks are nonzero. For the exogenous disturbances and set point trajectory, a fictitious uncertainty block $\Delta_{P}$ is introduced and robust stability is checked for the uncertainty block

$$
\left[\begin{array}{cccc}
\Delta_{P} & & & \\
& \Delta_{F B} & & \\
& & \Delta_{z d} & \\
& & & \Delta_{F O R}
\end{array}\right] .
$$

\section{V-A. PERTURBED MODEL OF A SRF CAVITY}

The perturbed model of a SRF cavity due to RF parameter variations is investigated in section IV-B. In the perturbed model of the SRF cavity, the Lorentz Force Detuning $\Delta \omega_{L}$ is treated as the perturbation of the system matrices. The microphonics are treated in the same way as the Lorentz Force Detuning. Also, the cavity RF parameter perturbations are treated as the system matrix perturbation. Figure 5.6 represents the generalized closed loop system with perturbed SRF cavity. Inthe intermediate configuration shown in figure 5.7, inputs and outputs of the generalized system are extracted. The inputs are

$$
\left\lfloor w: w_{F O R} \quad w_{\tau d} \quad w_{F B}: \tilde{d}_{R} \quad \tilde{d}_{B}: \begin{array}{lllll} 
& : & : & u_{C}
\end{array}\right\rfloor
$$

and the outputs are

$$
\left[\begin{array}{llllllllll}
v & : & z_{F O R} & z_{\tau d} & z_{F B} & : & y & \tilde{e}^{k} & : & e^{k}
\end{array}\right] .
$$

Figure 5.8 shows the generalized system whose inputs and outputs are given by (5.14) and (5.15). Now, from the configuration given in figure 5.7, the uncertainty block can be extracted.

$$
\left[\begin{array}{cccc}
\Delta_{S R F} & & & \\
& \Delta_{F B} & & \\
& & \Delta_{\tau d} & \\
& & & \Delta_{F O R}
\end{array}\right]
$$

and so the robust stability problem is investigated in the configuration shown in figure 5.9 where the uncertainty blocks are nonzero and the exogenous disturbances are zero and the robust stability is checked for the uncertainty block (5.16).

The robust performance problem is investigated in the configuration shown in figure 5.10 where the uncertainty blocks are nonzero and for the exogenous disturbances, set point trajectory, a fictitious uncertainty block $\Delta_{P}$ is introduced and robust stability is checked for the uncertainty block

$$
\left[\begin{array}{lllll}
\Delta_{P} & & & & \\
& \Delta_{S R F} & & & \\
& & \Delta_{F B} & & \\
& & & \Delta_{\tau d} & \\
& & & & \Delta_{F O R}
\end{array}\right] .
$$




\section{APPENDIX. PRoOf OF (3.8) AND (3.9)}

The transformation is defined by (4.7).

$$
\left[\begin{array}{c}
z \\
z_{\bar{o}}
\end{array}\right]=\left[\begin{array}{c}
z_{1} \\
z_{2} \\
z_{\bar{o} 1} \\
z_{\bar{o} 2}
\end{array}\right]=P x=\left[\begin{array}{c}
C\left(\Delta \omega_{L}\right) \\
v_{1} \\
v_{2}
\end{array}\right]\left[\begin{array}{l}
x_{1} \\
x_{2} \\
x_{3} \\
x_{4}
\end{array}\right]
$$

Let $V=\left[\begin{array}{l}v_{1} \\ v_{2}\end{array}\right]$. By the transformation (A.1), the system as given in (3.5) and (3.6) is transformed into

$$
\begin{aligned}
& {\left[\begin{array}{c}
\dot{z} \\
\dot{z}_{\bar{o}}
\end{array}\right]=\left[\begin{array}{cc}
\bar{A}_{o} & 0 \\
\bar{A}_{21} & \bar{A}_{\bar{o}}
\end{array}\right]\left[\begin{array}{c}
z \\
z_{\bar{o}}
\end{array}\right]+\left[\begin{array}{c}
B_{o} \\
B_{\bar{o}}
\end{array}\right] u+\left[\begin{array}{c}
B_{o I} \\
B_{\bar{o} I}
\end{array}\right] I} \\
& y=\left[\begin{array}{ll}
\bar{C}_{o} & 0
\end{array}\right]\left[\begin{array}{c}
z \\
z_{\bar{o}}
\end{array}\right]
\end{aligned}
$$

We need to show

$$
\begin{aligned}
& \bar{A}_{o}=A_{z}\left(\Delta \omega_{L}\right)=C\left(\Delta \omega_{L}\right) A\left(\Delta \omega_{L}\right) C^{+}\left(\Delta \omega_{L}\right), \\
& B_{o}=B_{z}=C\left(\Delta \omega_{L}\right) B\left(\Delta \omega_{L}\right), \\
& B_{o I}=B_{z I}=C\left(\Delta \omega_{L}\right) B_{I}\left(\Delta \omega_{L}\right), \\
& \bar{C}_{o}=C_{z}=I_{2} .
\end{aligned}
$$

From (A.1),

$$
\begin{aligned}
& {\left[\begin{array}{c}
\dot{z} \\
\dot{z}_{\bar{o}}
\end{array}\right]=\left[\begin{array}{c}
C\left(\Delta \omega_{L}\right) \\
V
\end{array}\right] A\left(\Delta \omega_{L}\right)\left[\begin{array}{c}
C\left(\Delta \omega_{L}\right) \\
V
\end{array}\right]^{-1}\left[\begin{array}{c}
z \\
z_{\bar{o}}
\end{array}\right]+\left[\begin{array}{c}
C\left(\Delta \omega_{L}\right) \\
V
\end{array}\right] B\left(\Delta \omega_{L}\right) u_{c}+\left[\begin{array}{c}
C\left(\Delta \omega_{L}\right) \\
V
\end{array}\right] B_{I}\left(\Delta \omega_{L}\right) I} \\
& y=C\left(\Delta \omega_{L}\right) x_{c}=C\left(\Delta \omega_{L}\right)\left[\begin{array}{c}
C\left(\Delta \omega_{L}\right. \\
V
\end{array}\right]^{-1}\left[\begin{array}{c}
z \\
z_{\bar{o}}
\end{array}\right] .
\end{aligned}
$$

From (A.2) and (A.8), we obtain

$$
\left[\begin{array}{c}
C\left(\Delta \omega_{L}\right) \\
V
\end{array}\right] A\left(\Delta \omega_{L}\right)\left[\begin{array}{c}
C\left(\Delta \omega_{L}\right) \\
V
\end{array}\right]^{-1}=\left[\begin{array}{cc}
\bar{A}_{o} & 0 \\
\bar{A}_{21} & \bar{A}_{\bar{o}}
\end{array}\right]
$$




$$
\begin{aligned}
& {\left[\begin{array}{c}
C\left(\Delta \omega_{L}\right) \\
V
\end{array}\right] B\left(\Delta \omega_{L}\right)=\left[\begin{array}{l}
B_{o} \\
B_{\bar{o}}
\end{array}\right],} \\
& {\left[\begin{array}{c}
C\left(\Delta \omega_{L}\right) \\
V
\end{array}\right] B_{I}\left(\Delta \omega_{L}\right)=\left[\begin{array}{l}
B_{o I} \\
B_{\bar{o} I}
\end{array}\right],} \\
& C\left(\Delta \omega_{L}\right)\left[\begin{array}{c}
C\left(\Delta \omega_{L}\right) \\
V
\end{array}\right]^{-1}=\left[\begin{array}{ll}
\bar{C}_{o} & 0
\end{array}\right] .
\end{aligned}
$$

First,

$$
\begin{aligned}
{\left[\begin{array}{c}
C\left(\Delta \omega_{L}\right) \\
V
\end{array}\right] A\left(\Delta \omega_{L}\right)\left[\begin{array}{c}
C\left(\Delta \omega_{L}\right) \\
V
\end{array}\right]^{-1}=\left[\begin{array}{cc}
\bar{A}_{o} & 0 \\
\bar{A}_{21} & \bar{A}_{\bar{o}}
\end{array}\right] } & \Leftrightarrow\left[\begin{array}{c}
C\left(\Delta \omega_{L}\right) \\
V
\end{array}\right] A\left(\Delta \omega_{L}\right)=\left[\begin{array}{cc}
\bar{A}_{o} & 0 \\
\bar{A}_{21} & \bar{A}_{\bar{o}}
\end{array}\right]\left[\begin{array}{c}
C\left(\Delta \omega_{L}\right) \\
V
\end{array}\right] \\
& \Leftrightarrow\left[\begin{array}{c}
C\left(\Delta \omega_{L}\right) A\left(\Delta \omega_{L}\right) \\
V A\left(\Delta \omega_{L}\right)
\end{array}\right]=\left[\begin{array}{c}
\bar{A} C\left(\Delta \omega_{L}\right) \\
\bar{A}_{21} C\left(\Delta \omega_{L}\right)+V \bar{A}_{\bar{o}}
\end{array}\right]
\end{aligned}
$$

Therefore,

$$
\bar{A}_{o}=A_{z}\left(\Delta \omega_{L}\right)=C\left(\Delta \omega_{L}\right) A\left(\Delta \omega_{L}\right) C^{+}\left(\Delta \omega_{L}\right) .
$$

It is trivial to show

$$
\begin{aligned}
& B_{o}=B_{z}=C\left(\Delta \omega_{L}\right) B\left(\Delta \omega_{L}\right), \\
& B_{o I}=B_{z I}=C\left(\Delta \omega_{L}\right) B_{I}\left(\Delta \omega_{L}\right) .
\end{aligned}
$$

Also,

$$
\begin{gathered}
C\left(\Delta \omega_{L}\right)\left[\begin{array}{c}
C\left(\Delta \omega_{L}\right) \\
V
\end{array}\right]^{-1}=\left[\begin{array}{ll}
\bar{C}_{o} & 0
\end{array}\right] \Leftrightarrow C\left(\Delta \omega_{L}\right)\left[\begin{array}{c}
C\left(\Delta \omega_{L}\right) \\
V
\end{array}\right]^{-1}=\left[\begin{array}{ll}
\bar{C}_{o} & 0
\end{array}\right]\left[\begin{array}{c}
C\left(\Delta \omega_{L}\right) \\
V
\end{array}\right] \\
\Leftrightarrow C\left(\Delta \omega_{L}\right)=\bar{C}_{o} C\left(\Delta \omega_{L}\right) .
\end{gathered}
$$

Hence,

$$
\bar{C}_{o}=C_{z}=C\left(\Delta \omega_{L}\right) C^{+}\left(\Delta \omega_{L}\right)=I_{2} .
$$




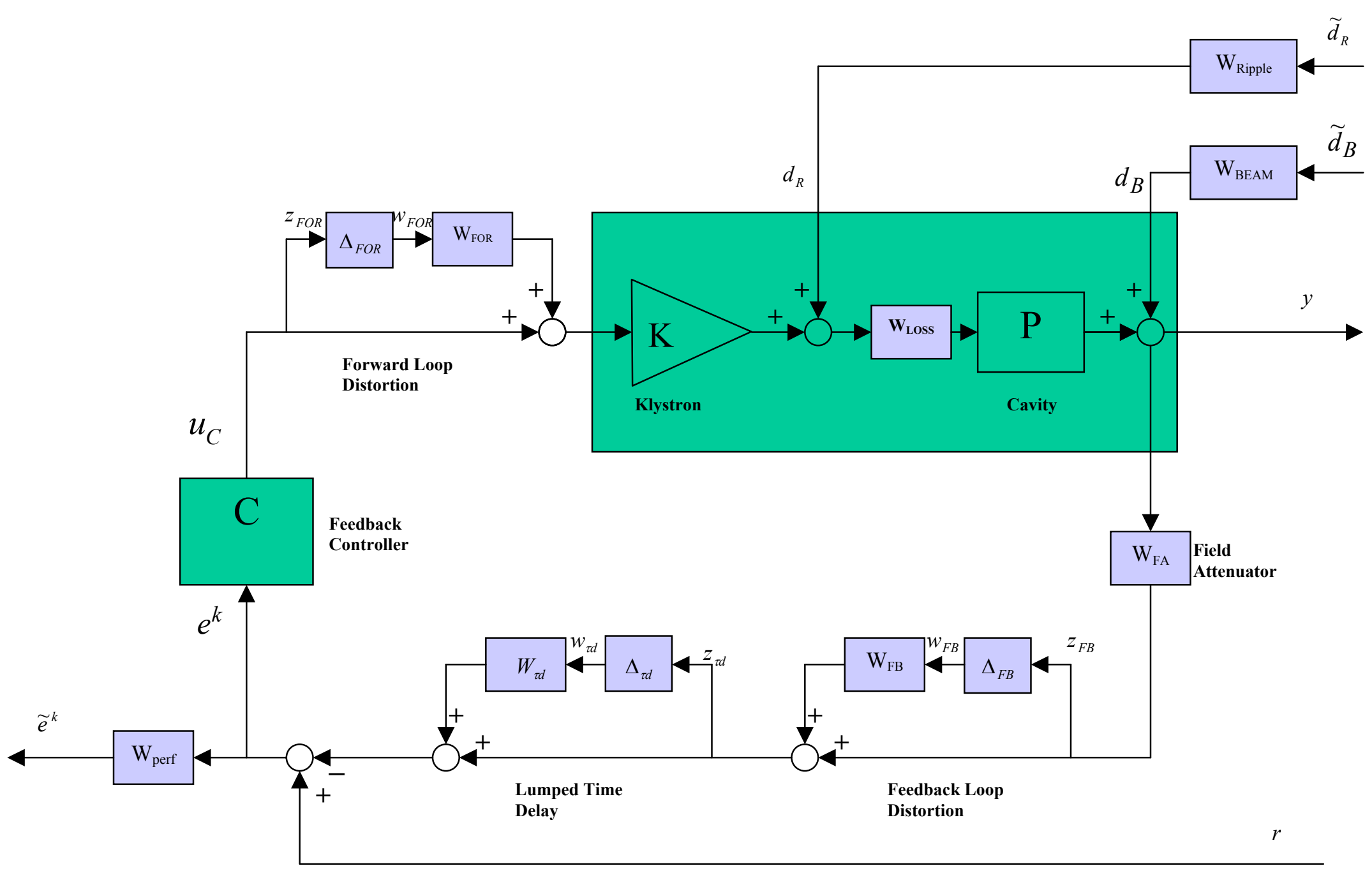

Figure 5.1 Perturbed Model of the Linear Accelerator RF system 


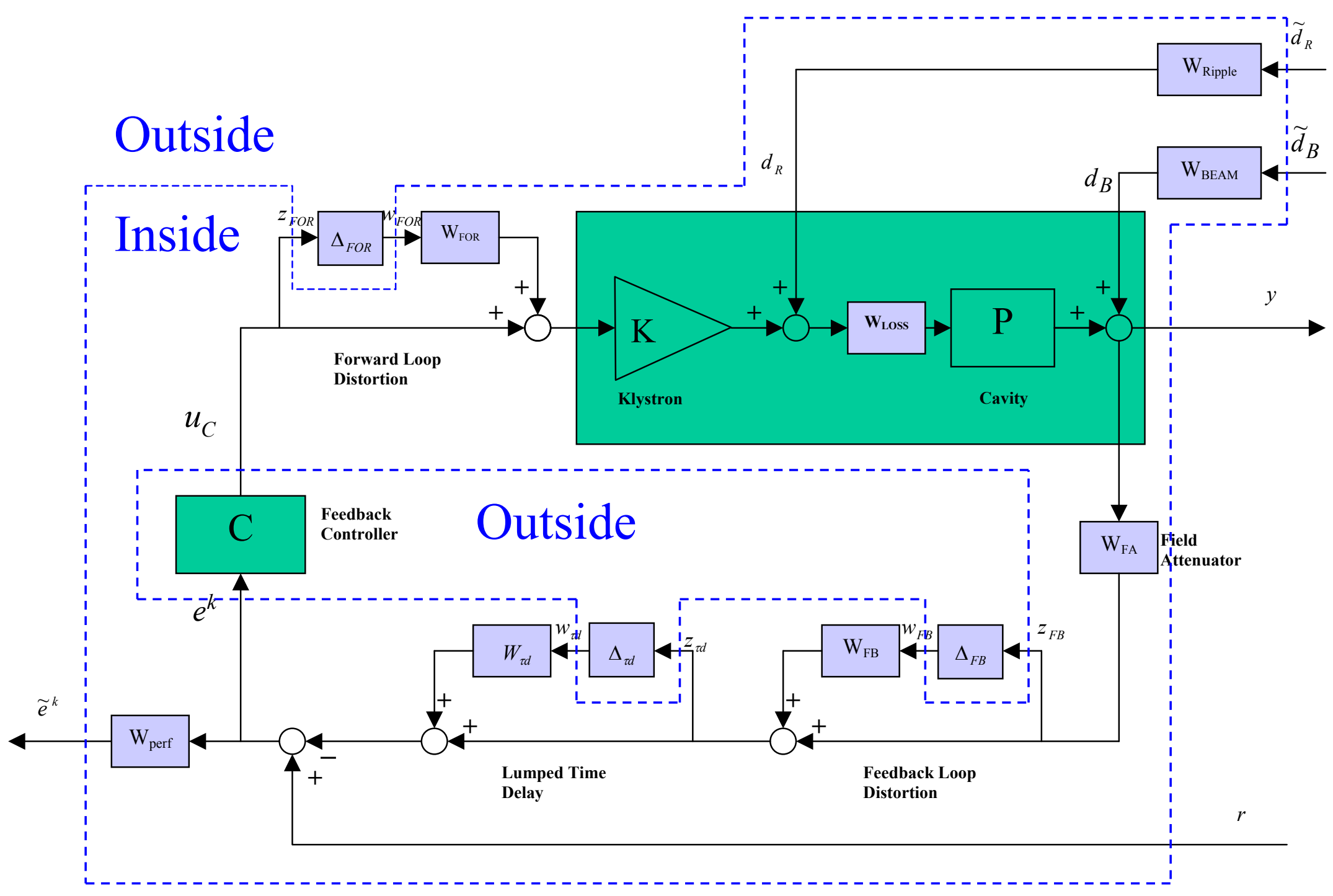

Figure 5.2 Perturbed Model of the Linear Accelerator RF system. Uncertainty Block, Open Loop Linear Fractional Transformation Interconnection, and Controller are to be extracted 


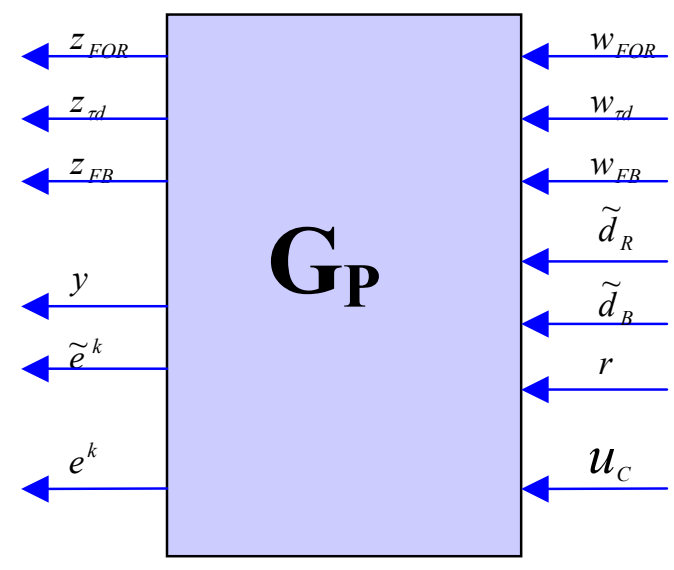

Figure 5.3 Linear Fractional Transformation Representation of Perturbed Open Loop System

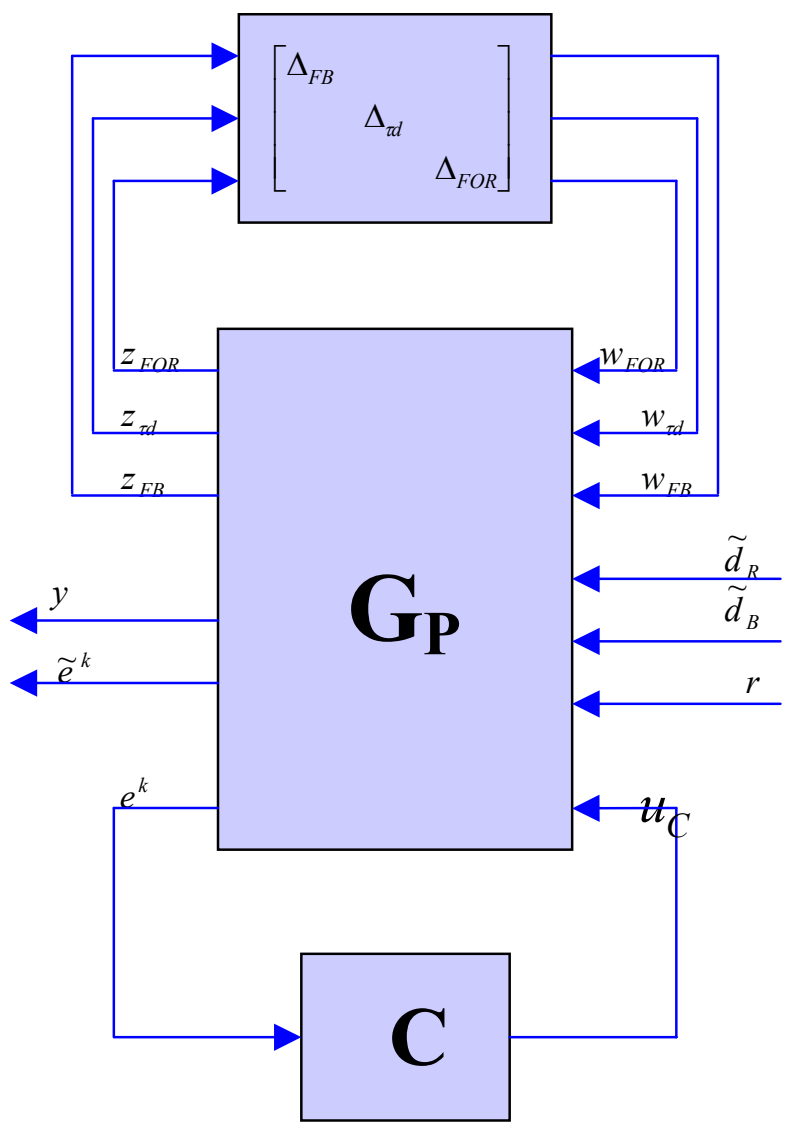

Figure 5.4 Linear Fractional Transformation Representation of Perturbed Closed Loop System 


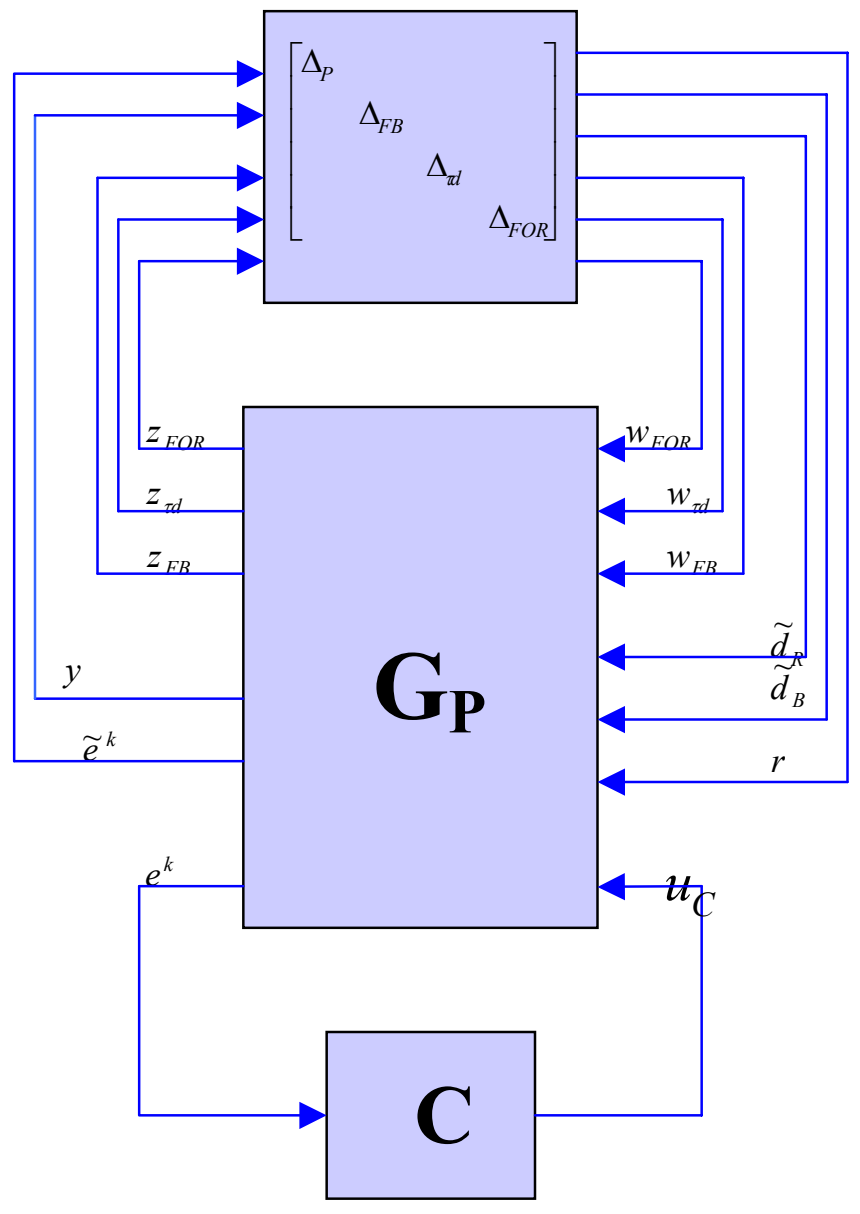

Figure 5.5 Linear Fractional Transformation Representation of Perturbed Closed Loop System for Robust Performance 


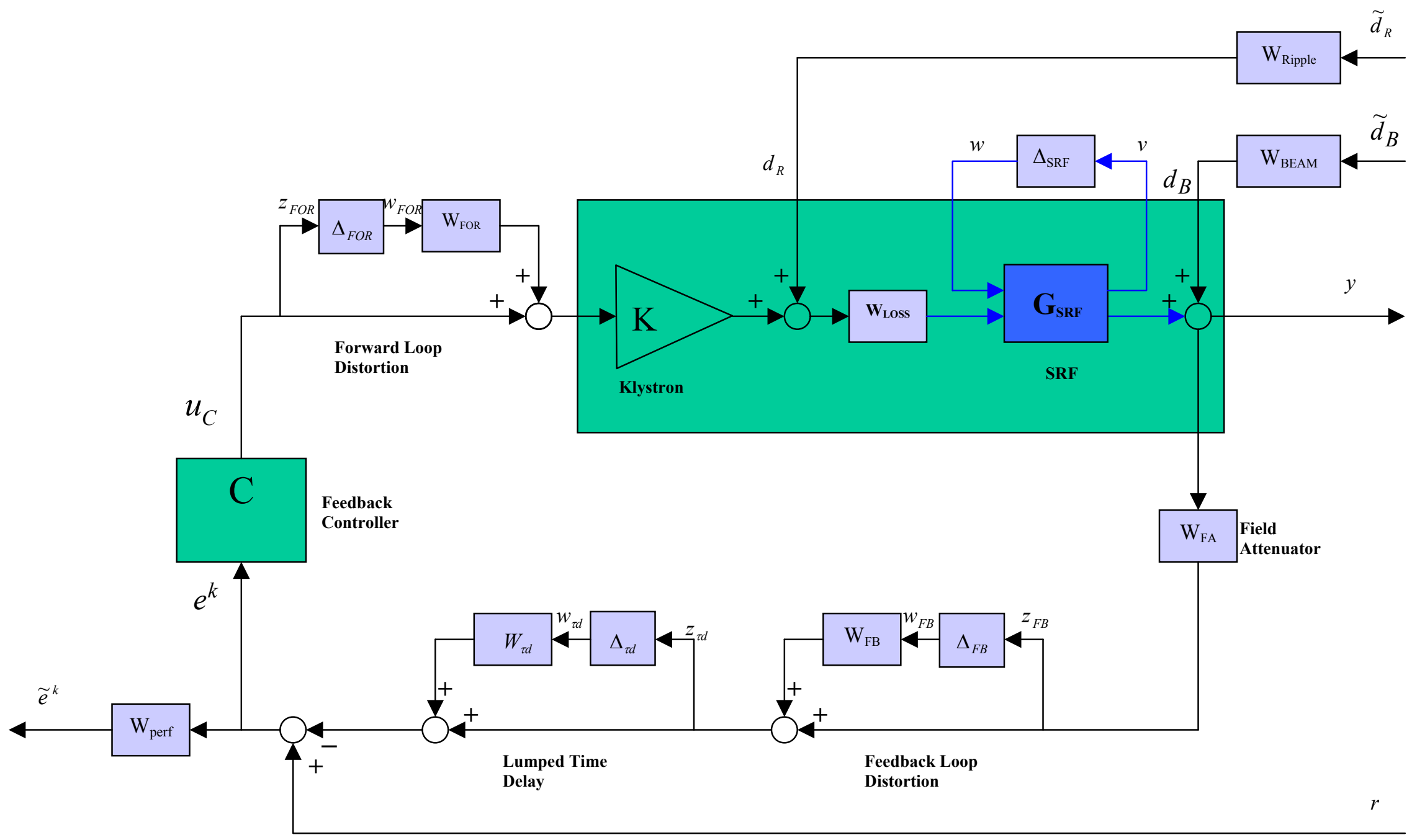

Figure 5.6 Perturbed Model of the linear accelerator RF system with the SRF Cavity 


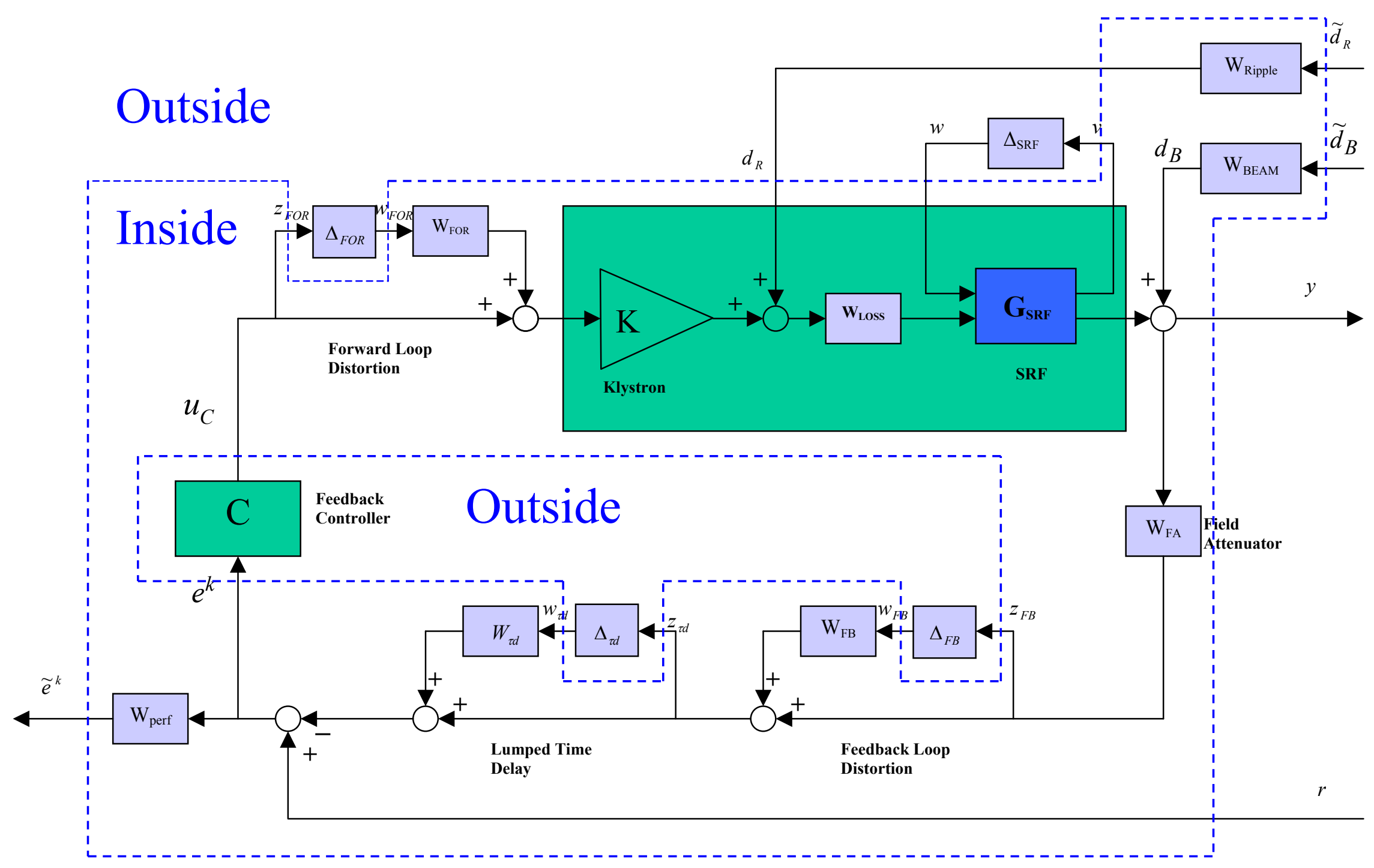

Figure 5.7 Perturbed Model of the Linear Accelerator RF system. Uncertainty Block, Open Loop Linear Fractional Transformation Interconnection, and Controller are to be extracted 


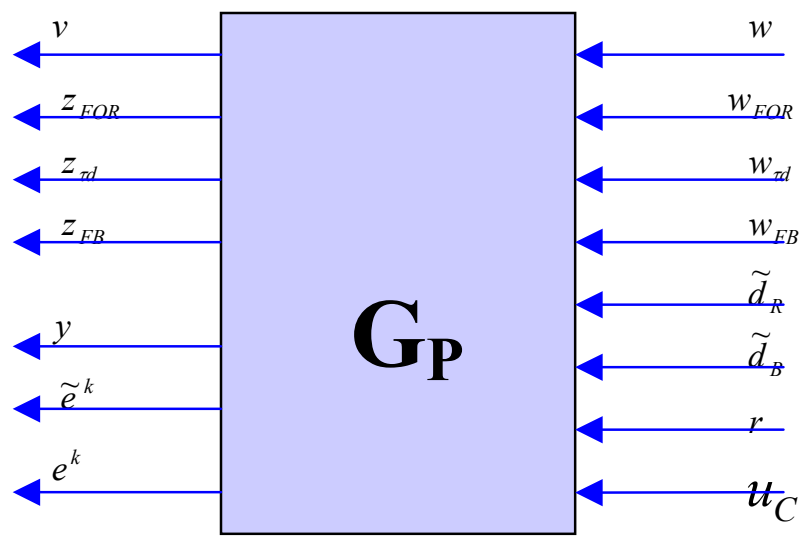

Figure 5.8 Linear Fractional Transformation Representation of Perturbed Open Loop System for SRF Cavity

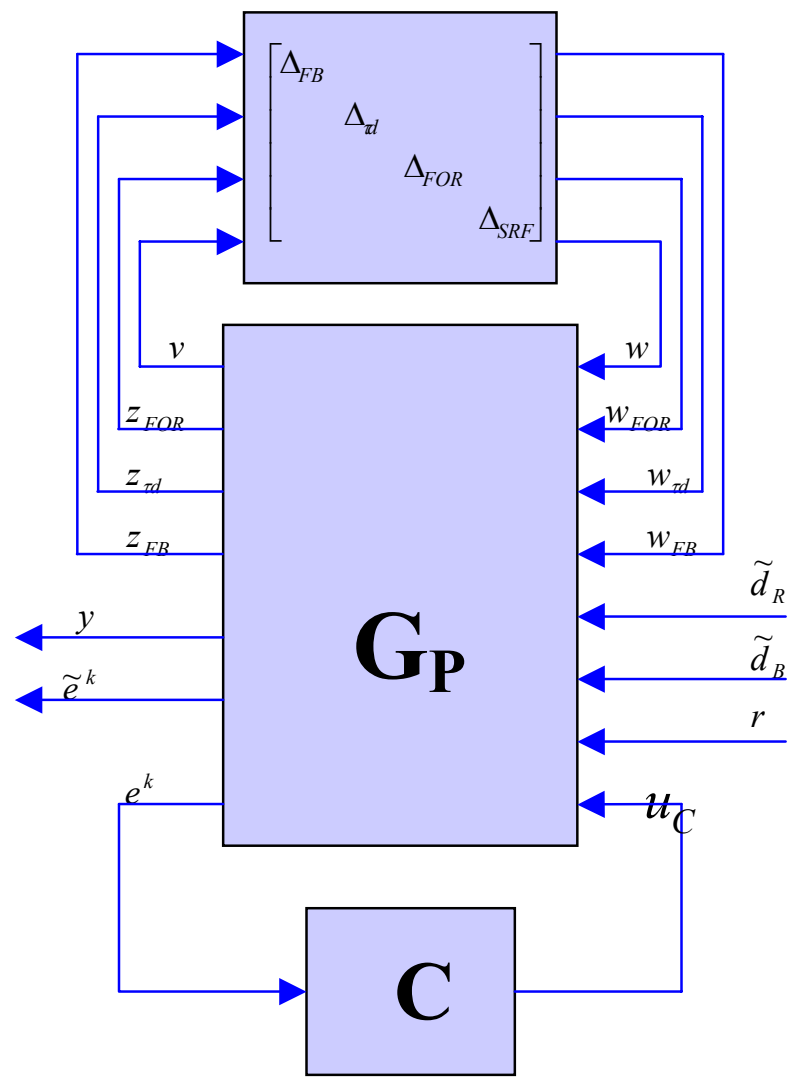

Figure 5.9 Linear Fractional Transformation Representation of Perturbed Closed Loop System for SRF Cavity 


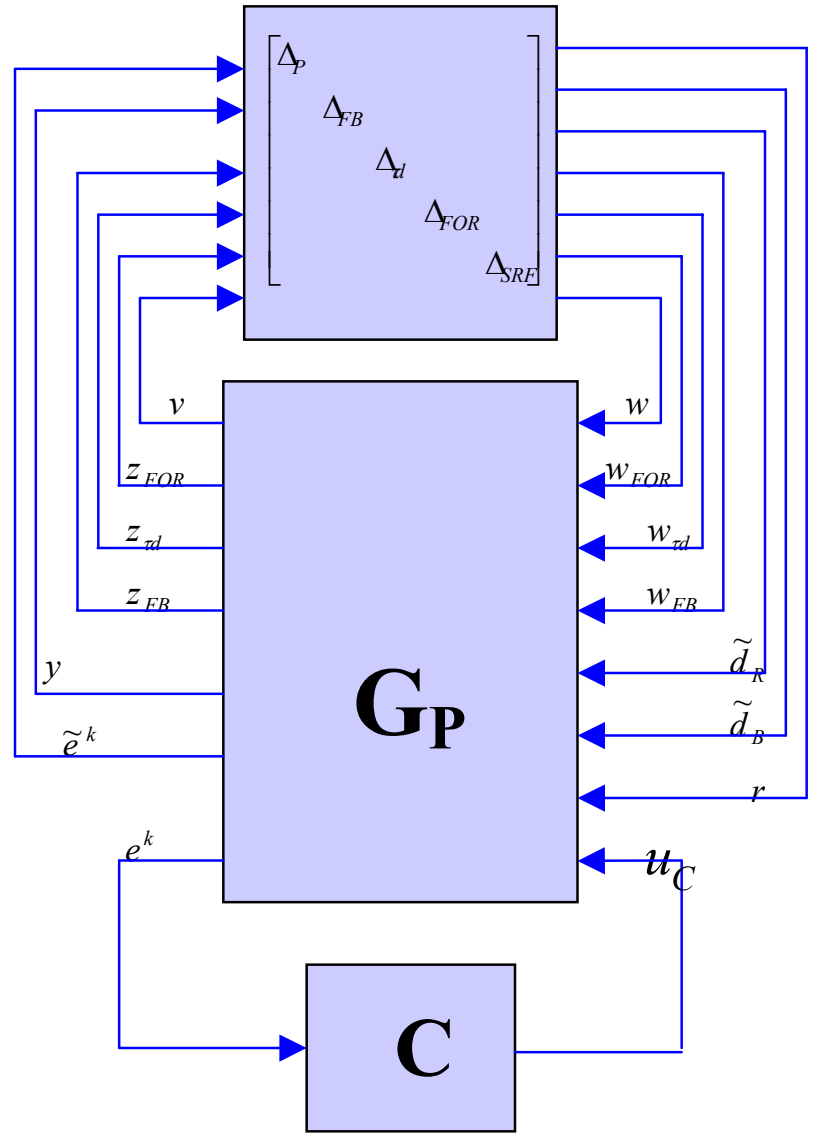

Figure 5.10 Linear Fractional Transformation Representation of Perturbed Closed Loop System for Robust Performance for SRF Cavity 